\title{
Review
}

\section{COVID-19: Review of a 21st Century Pandemic from Etiology to Neuro-psychiatric Implications}

Vicky Yamamoto ${ }^{\mathrm{a}, \mathrm{b}, \mathrm{c}, \mathrm{d}, 1}$, Joe F. Bolanos ${ }^{\mathrm{a}, \mathrm{b}, 1}$, John Fiallos ${ }^{\mathrm{a}, \mathrm{b}}$, Susanne E. Strand ${ }^{\mathrm{a}, \mathrm{b}}$, Kevin Morris ${ }^{\mathrm{a}, \mathrm{b}}$, Sanam Shahrokhinia ${ }^{\mathrm{e}}$, Tim R. Cushing ${ }^{\mathrm{f}}$, Lawrence Hopp ${ }^{\mathrm{g}}$, Ambooj Tiwari ${ }^{\mathrm{a}, \mathrm{h}}$, Robert Hariri ${ }^{\mathrm{a}, \mathrm{i}, \mathrm{j}}$, Rick Sokolov $^{\mathrm{k}}$, Christopher Wheeler ${ }^{\mathrm{a}, \mathrm{b}, \mathrm{l}}$, Ajeet Kaushik ${ }^{\mathrm{m}}$, Ashraf Elsayegh ${ }^{\mathrm{n}}$, Dawn Eliashiv ${ }^{\mathrm{a}, \mathrm{o}}$, Rebecca Hedrick ${ }^{\mathrm{p}}$, Behrouz Jafari ${ }^{\mathrm{q}}$, J. Patrick Johnson ${ }^{\mathrm{r}, \mathrm{s}}$, Mehran Khorsandi ${ }^{\mathrm{t}}$, Nestor Gonzalez ${ }^{\mathrm{s}}$, Guita Balakhani $^{\mathrm{u}}$, Shouri Lahiri ${ }^{\mathrm{v}}$, Kazem Ghavidel ${ }^{\mathrm{w}}$, Marco Amaya ${ }^{\mathrm{a}, \mathrm{b}}$, Harry Kloor ${ }^{\mathrm{a}}$, Namath Hussain ${ }^{\mathrm{a}, \mathrm{x}}$, Edmund Huang ${ }^{\mathrm{u}}$, Jason Cormier ${ }^{\mathrm{a}, \mathrm{y}}$, J. Wesson Ashford ${ }^{\mathrm{a}, \mathrm{z}}$, Jeffrey C. Wang ${ }^{\mathrm{a}, \mathrm{aa}}$, Shadi Yaghobian ${ }^{\mathrm{bb}}$, Payman Khorrami ${ }^{\mathrm{cc}}$, Bahman Shamloo ${ }^{\mathrm{dd}}$, Charles Moon ${ }^{\mathrm{ff}}$, Payam Shadi ${ }^{\mathrm{bb}}$ and Babak Kateb ${ }^{\mathrm{a}, \mathrm{b}, \mathrm{x}, \mathrm{ee}, \mathrm{gg}, *}$

${ }^{a}$ Society for Brain Mapping and Therapeutics (SBMT), Los Angeles, CA, USA

${ }^{\mathrm{b}}$ Brain Mapping Foundation (BMF), Los Angeles, CA, USA

${ }^{\mathrm{c}}$ USC Keck School of Medicine, The USC Caruso Department of Otolaryngology-Head and Neck Surgery, Los Angeles, CA, USA

${ }^{\mathrm{d}}$ USC-Norris Comprehensive Cancer Center, Los Angeles, CA, USA

${ }^{\mathrm{e}}$ Cedars-Sinai Medical Center, Department of Nutrition, Los Angeles, CA, USA

${ }^{\mathrm{f}}$ UCLA-Cedar-Sinai California Rehabilitation Institute, Los Angeles, CA, USA

${ }^{\mathrm{g}}$ Cedars Sinai Medical Center Department of Ophthalmology and UCLA Jules Stein Eye Institute,

Los Angeles, CA, USA

${ }^{\mathrm{h}}$ New York University, Department of Neurology, New York, NY, USA

${ }^{\mathrm{i}}$ Celularity Corporation, Warren, NJ, USA

${ }^{\mathrm{j}}$ Weill Cornell School of Medicine, Department of Neurosurgery, New York, NY, USA

${ }^{\mathrm{k}}$ Cedars-Sinai Medical Center, Department of Infectious Disease Los Angeles, CA, USA

${ }^{1}$ T-NeuroPharma, Albuquerque, NM, USA

${ }^{\mathrm{m}}$ NanoBioTech Laboratory, Department of Natural Sciences, Division of Sciences, Arts, and Mathematics, Florida Polytechnic University, Lakeland, FL, USA

${ }^{\mathrm{n}}$ Cedars Sinai Medical Center, Department of Pulmonology, Los Angeles, CA, USA

${ }^{\circ}$ UCLA David Geffen, School of Medicine, Department of Neurology, Los Angeles, CA, USA

${ }^{\mathrm{p}}$ Cedars Sinai Medical Center, Department of Psychiatry, Los Angeles, CA, USA

${ }^{\mathrm{q}}$ University of California, Irvine, School of Medicine, Department of Medicine, Irvine, CA, USA

${ }^{\mathrm{r}}$ Cedars Sinai Medical Center, Spine Institute, Los Angeles, CA, USA

${ }^{\mathrm{s}}$ Cedars-Sinai Medical Center, Department of Neurosurgery, Los Angeles, CA, USA

${ }^{\mathrm{t}}$ Cedars-Sinai Medical Center, Department of Cardiology, Los Angeles, CA, USA

\footnotetext{
${ }^{1}$ These authors contributed equally to this work.

${ }^{*}$ Correspondence to: Babak Kateb MD, Society for Brain Mapping and Therapeutics (SBMT), Los Angeles, CA, USA. E-mail:

Babak.Kateb@WorldBrainMapping.org.
} 
${ }^{\mathrm{u}}$ Cedars-Sinai Medical Center, Department of Nephrology, Los Angeles, CA, USA

${ }^{\mathrm{v}}$ Cedars-Sinai Medical Center, Department of Neurology, Los Angeles, CA, USA

${ }^{\mathrm{w}}$ University of Tehran School of Medicine, Department of Cardiology, Tehran, Iran

${ }^{\mathrm{x}}$ Loma Linda University, Department of Neurosurgery, Loma Linda, CA, USA

${ }^{y}$ Lafayette Surgical Specialty Hospital, Lafayette, Louisiana, USA

${ }^{\mathrm{z}}$ Stanford University School of Medicine (Affiliated), Department of Psychiatry and Behavioral Science and Department of Veteran's Affair, Palo Alto, CA, USA

${ }^{a}$ USC-Keck School of Medicine, Department of Orthopedic Surgery, Los Angeles, CA, USA

${ }^{\mathrm{bb}}$ Cedars-Sinai Medical Center, Department of Internal Medicine, Los Angeles, CA, USA

${ }^{\mathrm{cc} C e d a r s ~ S i n a i ~ M e d i c a l ~ C e n t e r, ~ D e p a r t m e n t ~ o f ~ G a s t r o e n t e r o l o g y, ~ L o s ~ A n g e l e s, ~ C A, ~ U S A ~}$

${ }^{\mathrm{dd}}$ Cedars Sinai Medical Center, Pain Management, Los Angeles, CA, USA

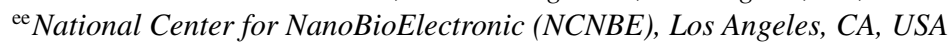

${ }^{\mathrm{ff}}$ Cedars Sinai Orthopaedic Center, Department of Orthopedics, Los Angeles, CA, USA

${ }^{\mathrm{gg}}$ Brain Technology and Innovation Park, Los Angeles, CA, USA

Accepted 14 August 2020

\begin{abstract}
COVID-19 is a severe infectious disease that has claimed $>150,000$ lives and infected millions in the United States thus far, especially the elderly population. Emerging evidence has shown the virus to cause hemorrhagic and immunologic responses, which impact all organs, including lungs, kidneys, and the brain, as well as extremities. SARS-CoV-2 also affects patients', families', and society's mental health at large. There is growing evidence of re-infection in some patients. The goal of this paper is to provide a comprehensive review of SARS-CoV-2-induced disease, its mechanism of infection, diagnostics, therapeutics, and treatment strategies, while also focusing on less attended aspects by previous studies, including nutritional support, psychological, and rehabilitation of the pandemic and its management. We performed a systematic review of $>1,000$ articles and included 425 references from online databases, including, PubMed, Google Scholar, and California Baptist University's library. COVID-19 patients go through acute respiratory distress syndrome, cytokine storm, acute hypercoagulable state, and autonomic dysfunction, which must be managed by a multidisciplinary team including nursing, nutrition, and rehabilitation. The elderly population and those who are suffering from Alzheimer's disease and dementia related illnesses seem to be at the higher risk. There are 28 vaccines under development, and new treatment strategies/protocols are being investigated. The future management for COVID-19 should include B-cell and T-cell immunotherapy in combination with emerging prophylaxis. The mental health and illness aspect of COVID-19 are among the most important side effects of this pandemic which requires a national plan for prevention, diagnosis and treatment.
\end{abstract}

Keywords: Alzheimer's disease, coronavirus, COVID-19, COVID-19 diagnostics, COVID-19 test, mental health, SARSCoV-2, SARS-CoV-2 treatment, Remdesivir, Hydroxychloroquine, Avigan, Actemra, COVID-19 Nutrition, COVID-19 Rehabilitation, COVID-19 Clinical manifestations, COVID-19 Risk factors and comorbidities, COVID-19 Vaccines, COVID19 immunotherapy, COVID-19 Natural Killer Cells and stem cell therapy, COVID-19 Janus Kinase Inhibitor 1 and 2, COVID-19 air filtration, Nanomedicine for COVID-19, COVID-19 mental disorders, COVID-19 domestic abuse, COVID-19 Alzheimer's Disease and dementia, COVID-19 complications, COVID-19 Screening, COVID-19 ophthalmology, COVID-19 Neurological implications, COVID-19 antiviral, COVID-19 Steroids, COVID-19 Convalescent plasma, COVID-19 anticoagulants, COVID-19 monoclonal antibodies

\section{INTRODUCTION}

The report of the World Health Organization (WHO) states that the pandemic of SARS-CoV-2 has registered 15,785,641 confirmed cases globally and 640,016 deaths as of July 26, 2020 [1].
The first case of the Coronavirus Disease 2019 (COVID-19) was reported by Dr. Li Wenliang, an ophthalmologist in Wuhan China in December 2019. This respiratory disease presented similar symptoms to severe acute respiratory syndrome (SARS-CoV) in 2003. It was later named Severe Acute Respira- 
tory Syndrome Coronavirus 2 (SARS-CoV-2) [2]. COVID-19 affects every age group; however, the elderly are more prone to a severe symptom display and have a higher mortality rate. Even with the majority of affected people being under the age of $65(83.2 \%)$, people over the age of 65 still stands for $79.5 \%$ of all COVID-19 related deaths in the United States (US) [3]. An average of $13.3 \%$ of people over the age of 65 have a proven neurological comorbidity, including dementia [4]. Although the elderly population is most severely affected by the illness, a new study has found a higher SARS-CoV-2 viral RNA load in young children's nasopharynx than what had previously been found in older children (>5 years old) and adults [5]. Young children might therefore be far more likely to spread the virus than the anticipated asymptomatic spreaders. In other words, individuals of all age groups are prone to both contracting and spreading the virus, even when mild or no symptoms are present [6].

In recent months many papers have been published, criticized, discredited, and retracted. Thus, we believe this comprehensive review could update scientists about the state-of-the-science and technology and clarify any ambiguity about the current diagnostics and therapeutics strategies for COVID-19.

\section{METHODOLOGY}

As the COVID-19 pandemic surges worldwide, new information on the virus and its consequences are being published daily. We carried out a comprehensive review of available data to gather relevant information on the SARS-CoV-2 infection and organized it in a single article. Four primary investigators, VY, JB, JF, and SES, conducted searches for pertinent information published on the disease. In this paper, we focus on the virology, clinical features, risk factors and comorbidities, clinical manifestations, diagnosis, treatment, mental health, nutrition, rehabilitation, and general complications of COVID-19.

\section{Search}

Over 1,000 research articles, clinical studies, and news outlet sources with data and information about COVID-19 were assessed. Of the total number of articles, 425 individual records have been included in this review. No additional articles were retrieved after screening the references in both included reports and excluded reviews.

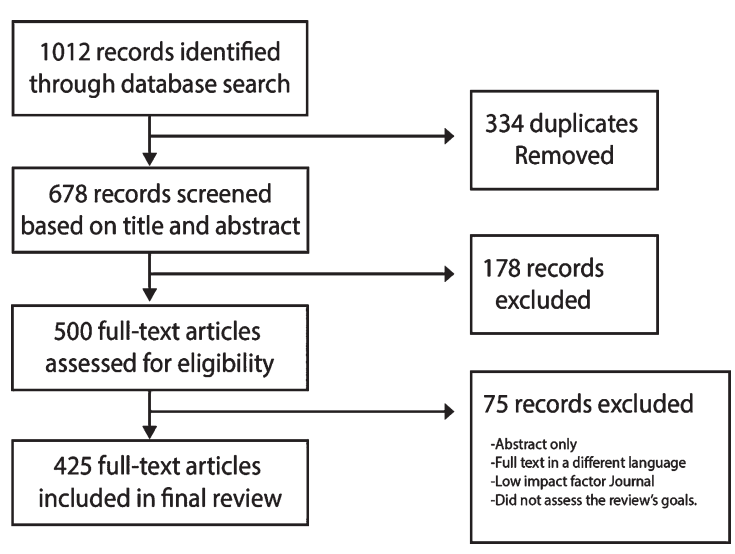

Databases used in the search for articles included PubMed, California Baptist University Library, ClinicalTrials.gov, Google Scholar, and multiple news sources. The search was restricted to articles about COVID-19 in English published between December 1, 2019 to August 4, 2020. We only considered articles for inclusion published in reputable journals with a high impact factor that pertained to the topic and investigational inquiries, as well as rigorous methodology.

Each article included was selected and reviewed by the 35 authors who then provided comments based on their area of expertise. Research papers were reviewed and critiqued independently; those not meeting the requirement for inclusion were excluded. Part of this data presented in this research paper is in development and not yet published, hence the need to convene a panel of COVID-19 treating specialists.

Keywords for the search included "COVID-19", "Coronavirus", "Novel Coronavirus Complications", "COVID-19 Treatments, Epidemiology", "SARSCoV-2", "COVID-19, mental health", "COVID19 neurology”, “ADRD”, "Alzheimer's Disease", "Long-term-care residents", "COVID-19 renal", "COVID-19 ophthalmology", "COVID-19 rehabilitation", "COVID-19 nutritional support".

Articles on the origins of coronaviruses from the 1980s until the most recent ones were also included as additional background information for this article.

\section{VIROLOGY OF COVID-19}

Coronaviruses (CoVs) are enveloped, nonsegmented, positive-sense, single-stranded, RNA viruses; belonging to the family Coronaviridae, broadly distributed in humans and other mammals [7]. 
Even though most coronavirus diseases are typically mild, two previous outbreaks in the past decades, SARS-CoV and the Middle East Respiratory Syndrome CoV (MERS-CoV), were severe. Collectively, SARS-CoV and MERS-CoV have produced more than ten thousand cumulative cases with a mortality rate of approximately $10 \%$ and $37 \%$, respectively $[7,8]$.

The WHO recently classified SARS-CoV-2 as a $\beta$-Coronavirus, its genetic sequence displayed more than $80 \%$ similarity to SARS-CoV and $50 \%$ to MERS-CoV, both having their origins in bats [8]. Transmission since early on in the SARS-CoV-2 outbreak has been primarily from human-to-human, via direct contact or droplets expelled by sneezing or coughing from an infected individual at close range. Some fecal-oral transmission has been recognized [8, 13]. Prenatal infection of mothers has not shown to result in fetal or newborn infections $[10,11]$. The mean reproductive number (R0) based on clinical data of patients for COVID-19 was 2.24-3.58 [11], indicating that each infected individual, on average, would spread the disease to $\sim 2-3$ other people $[12,13]$.

The virion of SARS-CoV-2 is approximately 50-200 nm in diameter [13]. It has at least four known structural proteins: spike (S) protein, envelope (E) protein, membrane $(\mathrm{M})$ protein, and nucleocapsid (N) protein (see Fig. 1). All are required to assemble a complete viral particle. Recent studies have shown that some CoVs do not need all four proteins to be an infectious virion, suggesting that other proteins with overlapping functionality may also be encoded [15].

The $\mathrm{S}$ protein is responsible for promoting host attachment and virus-target cell membrane fusion during virus infection $[15,16]$. This protein has three segments that form a crown-like structure on the envelope, from which the family name derives: corona is Latin for crown [30]. The portions are constituted by a single-pass trans-membrane anchor, a short intracellular tail, and a large ectodomain, which consists of an $\mathrm{S} 1$ receptor-binding subunit $\mathrm{S} 1$ and a membranefusion subunit $\mathrm{S} 2$. The $\mathrm{S} 1$ portion binds to an ACE2 receptor and the serine protease TMPRSS2 on the host cell surface for viral attachment [29], the S2 portion mediates the fusion of the host and viral membranes, allowing viral genomes to enter host cells [17-19].

Earlier this year [20], the ACE2 receptor was recognized as the SARS-CoV-2 receptor, which is present in multiple human tissues, including type I and type II alveolar epithelial cells in the lung, in the gastrointestinal tract, in the basal cell layer of epidermis and hair follicles [21]. Also present at the cellular level in the central nervous system [22,23], and in the kidneys and testes [24].

The SARS-CoV-2 S protein shares around $76 \%$ amino acids with the SARS-CoV and MERS protein [25], which may help explain its relatively high binding affinity to the human ACE2 receptor [23].

The $\mathrm{N}$ protein is attached to the CoV RNA genome and builds the nucleocapsid, which is part of the viral structure.

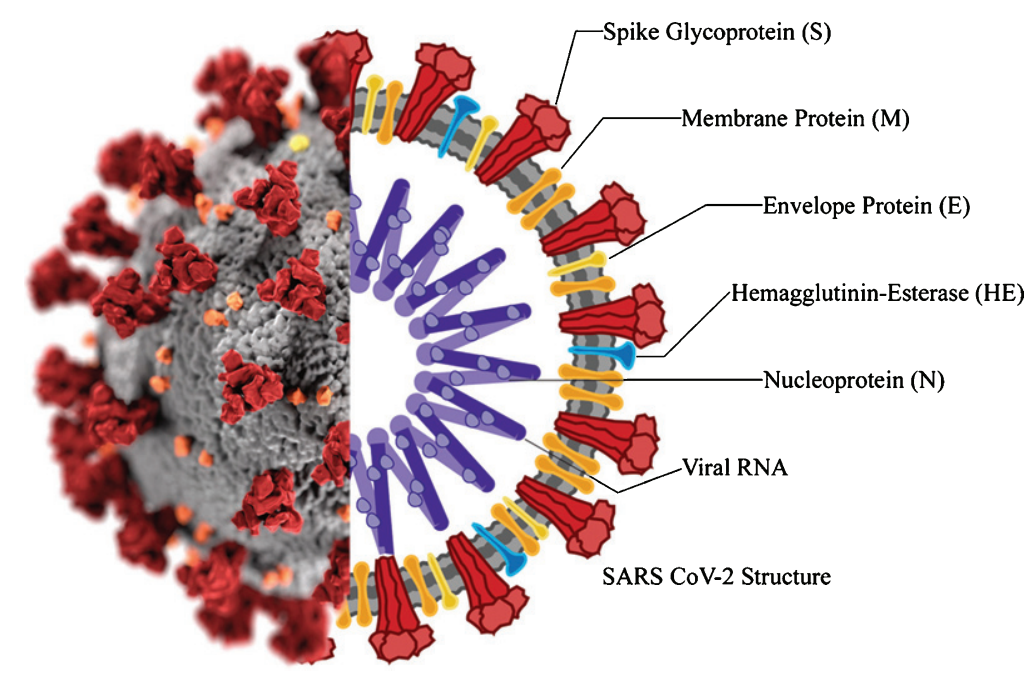

Fig. 1. Illustration of COVID-19 Structure. Adapted from the CDC images library [27] and Encyclopedia Britannica [28]. Illustrated by Dr. Joe Bolanos. 
The $\mathrm{M}$ protein is the most abundant structural protein in the viral envelope. It interacts with all the major structural proteins in the $\mathrm{CoV}$ assembly process. The necessary interaction of $\mathrm{S}$ and $\mathrm{M}$ proteins maintains the $S$ protein in the endoplasmic reticulum/Golgi complex for integration into new virions. The combination of the $\mathrm{M}$ and $\mathrm{E}$ protein consists to the structure of the viral envelope.

The $\mathrm{E}$ protein is the smallest of the structural proteins (containing 76-109 amino acids, in the range of 8.4 to $12 \mathrm{kDa}$ in size), but it is the most important for viral replication [15]. During replication, the $\mathrm{E}$ protein is upregulated on the infected cell endoplasmic reticulum, and only a small quantity is incorporated into the new virion envelope. Studies have shown that recombinant CoVs that lack the E protein exhibit reduced viral titters and impaired viral maturation [15]. The $\mathrm{CoV} E$ protein has a short, hydrophilic amino terminus comprised of 7-12 amino acids. This is followed by a large hydrophobic transmembrane domain consists of 25 amino acids, and ending with an extended, hydrophilic carboxyl terminus that constitutes the majority of the protein. The transmembrane domain has two neutral amino acids: valine and leucine, that account for the E protein's hydrophobicity [26] (Fig. 2). The SARS-CoV E protein has a PDZ binding motif located in the last four amino acids of the $C$ terminus. On co-immunoprecipitation and pull-down assays, the SARS-CoV E PDZ domain binds to PALS1 in mammalian cells, a tight junction-associated protein crucial for the establishment and maintenance of epithelial polarity in mammals [25]. Such functions and interactions exhibit multiple ways in which the $\mathrm{E}$ protein critically mediates SARS-CoV pathogenesis [25].

Analyses of 103 genetic populations of SARS$\mathrm{CoV}-2$ genomes showed that these viruses developed into two dominant strains, called $\mathrm{L}$ and $\mathrm{S}$. The $\mathrm{S}$ type is the original strain and was less aggressive and less prevalent than $\mathrm{L}$ ( $30 \%$ versus $70 \%$ of cases, respectively). The $\mathrm{L}$ type strain was already more frequent in the early stages of the Wuhan outbreak, but decreased in frequency after early January 2020 [31]. Later this year, further mutations were found, defining additional strains called A, B, and C [32]. Over time It is normal to collect random genomic mutations, which depend on age. Genomes develop variable mutations, constituting markers of disease spread. By building a phylogeny, it is possible to gather information about the epidemiological phenomena occurring to such pathogens, such as spread, their timeline of appearance and epidemic growth rate [33]. There are websites dedicated to tracking strains and updated daily, as the numbers of strains have increased [33]. Nextstrain.org identifies 5 major clades that correspond to GISAID nomenclature (in parenthesis): 19A (L) \& (V), 19B (S), 20A (G), 20B (GR), 20C (GH). Different methods of classification and nomenclature are in place [34]. The GISAID initiative has classified the major SARS-CoV-2 clades and named them according to marker mutations in 6 major phylogenetic groups starting from the initial split of $\mathrm{S}$ and $\mathrm{L}$, to the later division of $\mathrm{L}$ into $\mathrm{V}$ and $\mathrm{G}$ and later of $\mathrm{G}$ into GH and GR. So far 6 major clades have been identified based on 9 marker variants [34]:.

- S: C8782T, T28144C includes NS8-L84S

- L: C241, C3037, A23403, C8782, G11083, G25563, G26144, T28144, G28882 (WIV04reference sequence)

- V: G11083T, G26144T NSP6-L37F+NS3G251

- G: C241T, C3037T, A23403G includes SD614G

- GH: C241T, C3037T, A23403G, G25563T includes S-D614G+NS3-Q57H

- GR: C241T, C3037T, A23403G, G28882A includes S-D614G+N-G204R [34].

Based on the data available from GISAID the Geographical worldwide distribution of COVID-19 clades (GISAID) is illustrated in Fig. 3.

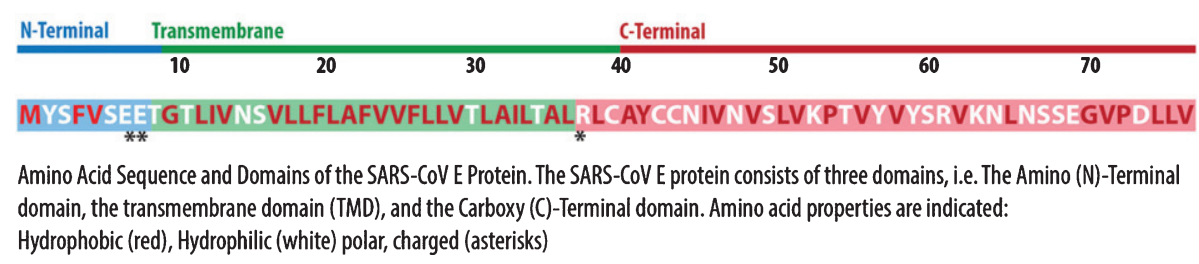

Fig. 2. Illustration SARS-CoV Protein E. Adapted from [15]. Illustrated by Dr. Joe Bolanos. 


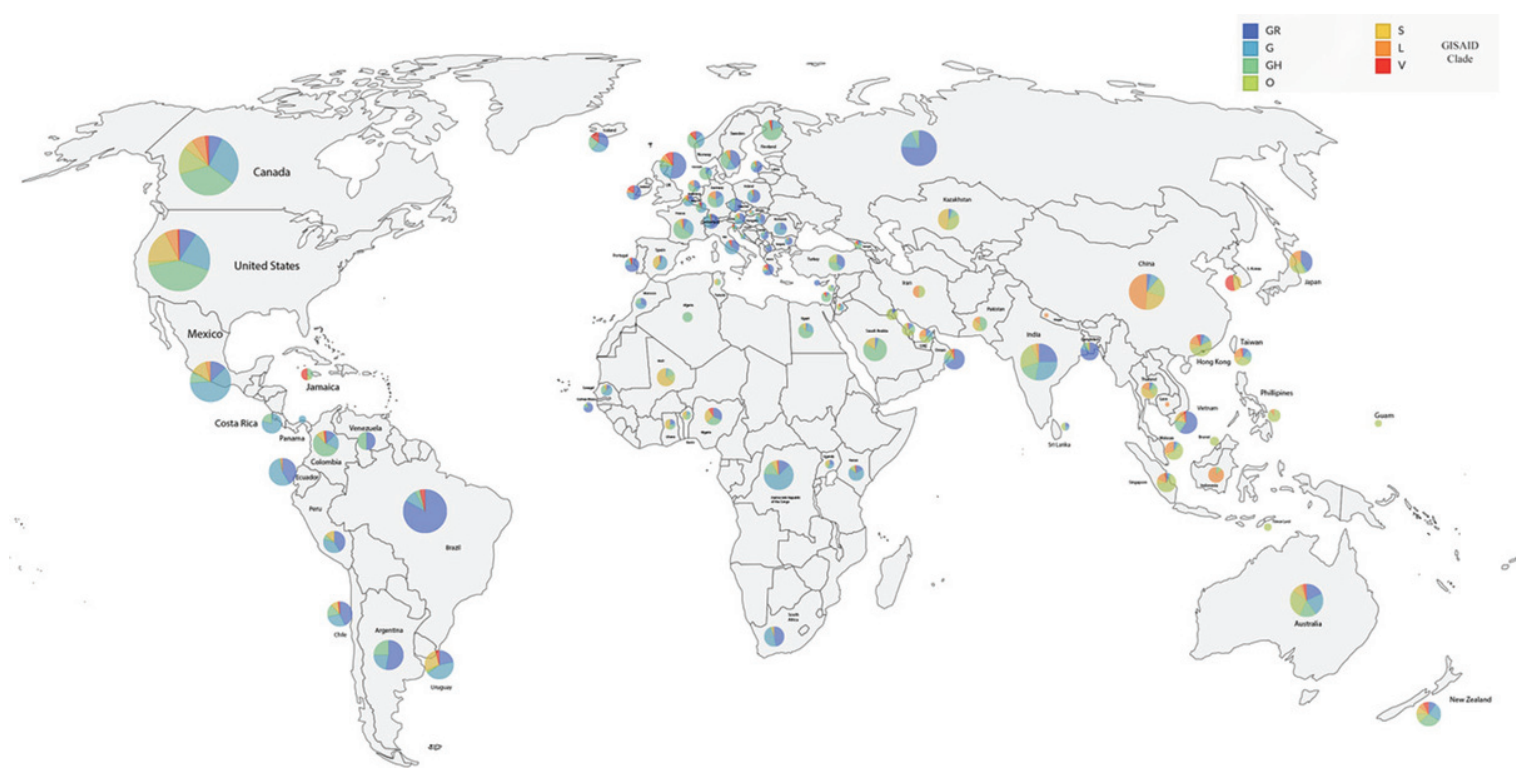

Fig. 3. Distribution of COVID-19 Worldwide distribution. Adapted from the GISAID Genomic Epidemiology of hCoV-19. Last updated 07-24-2020 https://nextstrain.org/ncov/global/gisaid?c=GISAID_clade\&p=full [35]. Illustrated by Dr. Joe Bolanos.

\section{CLINICAL FEATURES}

Symptoms of the virus appear after an average of 5.2 days. The period between the onset of symptoms until death ranges from 6 to 41 days, which depending on the age, underlying health complications, level of care, and immune system status. The time from symptomatic onset to death is typically shorter in those over 70 , median days (11.5 days) from the first symptom to death, than those with ages below 70 years ( 20 days) $[8,9]$.

The most common symptoms of COVID-19 in the US and China and most common laboratory findings of COVID-19 in China are shown in Fig. 4 [31, 43-45]. The Centers for Disease Control and Prevention (CDC) lists additional COVID-19 symptoms reported, including anosmia, ageusia, sore throat, and muscle pain [36], skin rashes and acro-ischemic lesions of the feet (anecdotally called "COVID toes") and hands are reported [37-39]. Children tend to exhibit milder symptoms than adults [40-42].

\section{Risk factors and comorbidities}

Studies have revealed how patients who develop SARS- CoV-2 pneumonia also tended to have underlying medical conditions such as hypertension (12.8-30\%), diabetes (5.3-12.1\%), and cardiovascular disease $(4.2 \%)[46,47]$. Patients exhibiting severe

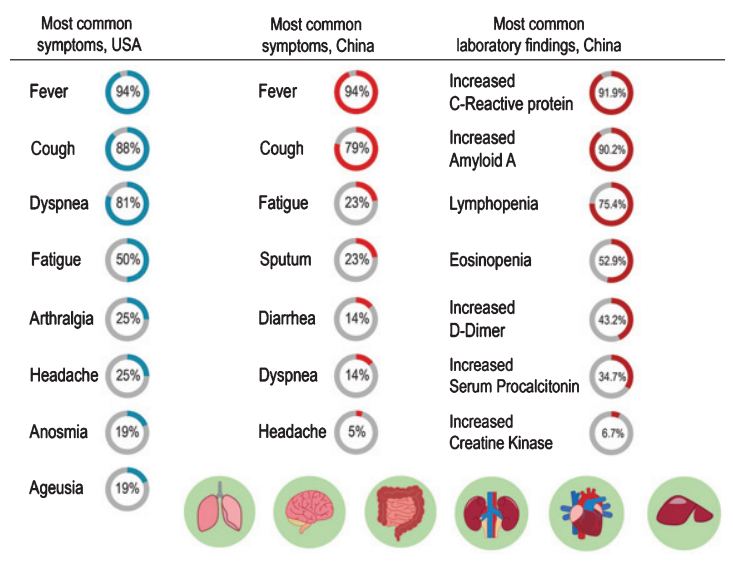

Fig. 4. Most common COVID-19 symptoms in US and China. Most common laboratory findings in COVID-19 in China [31, 43-45]. Illustrated by Dr. Joe Bolanos.

COVID-19 admitted to the intensive care unit (ICU) showed a greater tendency toward such comorbidities $[46,47]$. There are additional reports of comorbidities with lower frequencies.

The age group with the most cases was the sixth decade of life, followed by seventh decade, and then the fifth decade of life group [43]. Accordingly, hospitalization for patients in the seventh, eighth, and ninth decade of life are $11.8 \%, 16.6 \%$, and $18.4 \%$, respectively [43]. The groups with the most fatalities are the ninth, eighth, and seventh decades of life groups 
(shown in Graph 1). According to the CDC, as of July 2020 , there have been $3,186,778$ positive cases in the US. The largest age group presenting COVID19 cases is the 50-64 years old group (22.10\%), followed by the $65-74$ years old $(8.20 \%), 75-84$ years old $(4.9 \%)$, and $85+$ years old $(3.9 \%)$. Fatalities, however, show a different trend as illustrated in Graph 2.

\section{Clinical manifestations}

The clinical manifestations of COVID-19 range from asymptomatic or mild disease, to respiratory failure requiring mechanical ventilation and intensive care unit support, to widespread multi-organsystemic disease [48]. Acute respiratory distress

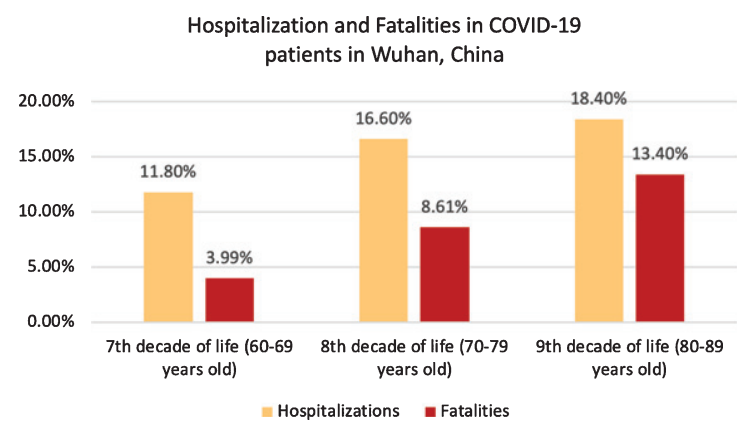

Graph 1. Hospitalizations and Fatalities in COVID-19 patients in Wuhan, China [43].

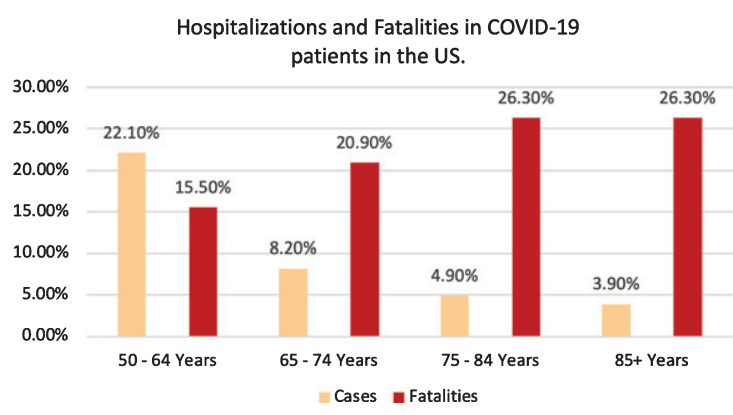

Graph 2. Hospitalizations and Fatalities in COVID-19 patients in the US [3].

syndrome (ARDS) trend prevalence is higher in the older age group, while a more significant percentile of heart, liver, and kidney function injuries were seen in the younger group [49]. Clinical presentation of patients with COVID-19 includes severe dyspnea and hypoxemia, renal impairment with decreased urine output, tachycardia, altered mental status, and multiple organ dysfunction. This clinical presentation is accompanied by altered laboratory values such as hyperbilirubinemia, acidosis, high lactate, coagulopathy, and thrombocytopenia [50].

The Chinese Center for Disease Control report has classified the disease by severity (see Table 1 ).

The Chinese guidelines for diagnosis and treatment of SARS-CoV-2 infection classify pneumonia caused by the novel coronavirus 2019 in four types. See Table $2[48,50]$.

Table 1

Adapted from [48] Classification of COVID-19 Pneumonia like symptoms

\begin{tabular}{|c|c|}
\hline Mild & Non-pneumonia and mild-pneumonia in approx. $81 \%$ of cases [48]. \\
\hline Severe & $\begin{array}{l}\text { Dyspnea, Respiratory Rate } \geq 30 \mathrm{rpm} \text {, blood oxygen saturation }(\mathrm{SpO} 2) \leq 93 \% \text {, } \\
\mathrm{PaO} 2 / \mathrm{FiO} 2 \text { ratio or } \mathrm{P} / \mathrm{F}<300 \text {, and/or lung infiltrates }>50 \% \text { within } 24 \text { to } 48 \text { hours. } \\
14 \% \text { of cases }[48] \text {. }\end{array}$ \\
\hline Critical & $\begin{array}{l}\text { Respiratory failure, septic shock, and/or multiple organ dysfunction (MOD) or } \\
\text { failure (MOF), which occurred in } 5 \% \text { of cases [48]. }\end{array}$ \\
\hline
\end{tabular}

Table 2

Adapted from [48, 50] Classification of severity of COVID-19

\begin{tabular}{|c|c|}
\hline Mild & $\begin{array}{l}\text { Usually presenting symptoms of an upper respiratory infection: mild fever, dry } \\
\text { cough, sore throat, nasal congestion, malaise, and headache, but no imaging signs } \\
\text { of pneumonia }[48,50] \text {. }\end{array}$ \\
\hline Moderate & $\begin{array}{l}\text { Presenting respiratory symptoms, such as cough and dyspnea (tachypnea in } \\
\text { children), and imaging signs of pneumonia }[48,50] .\end{array}$ \\
\hline Severe & $\begin{array}{l}\text { Presents with any of the following: } \\
\text { - Fever } \\
\text { - Severe dyspnea, respiratory distress, and tachypnea with } \mathrm{RR}>30 \mathrm{rpm} \\
\text { - Hypoxia }<90 \% \text {, or } \mathrm{PaO} 2 / \mathrm{FiO} 2<300 \mathrm{mmHg} \text {. } \\
\text { - Cyanosis could be present in children }[48,50]\end{array}$ \\
\hline $\begin{array}{l}\text { Critically } \\
\text { Severe }\end{array}$ & - Respiratory failure needing mechanical ventilation $[48,50]$ \\
\hline
\end{tabular}




\section{Ocular involvement}

Exposed ocular surfaces are vulnerable to infection by droplets as are other mucous membranes [51]. According to a study in 38 patients, in Hubei, 12 presented ocular symptoms. Ophthalmic manifestations from COVID-19 ranged from conjunctival hyperemia (3 patients), follicular conjunctivitis, chemosis (7 patients), and epiphora (7 patients) [52] to hyperreflective lesions in the ganglion cell and inner plexiform layers of the retina (12 patients, as reported in a study in Sao Paulo) as shown by optical coherence tomography [53]. COVID-19 hypercoagulable states have been correlated with blindness, due to acute ophthalmic artery occlusion [54].

The ocular surface could facilitate the virus to reach the nasopharynx through the nasolacrimal system [55]. Tears have been shown to harbor SARS$\mathrm{CoV}-2$ thus every healthcare worker needs to take caution as $7 \%$ of COVID-19 patients may present the virus in their tears, but be asymptomatic [56] and while studies have shown positive SARS-CoV-2 RT-PCR results from a patient's tears, virus isolation remains difficult [57].

\section{Neurological implications}

Human coronaviruses ( $\mathrm{HCoVs}$ ), and other respiratory viruses may enter the central nervous system (CNS) hematogenously or by the ethmoidal cribriform plate by retrograde neuronal route $[23,58]$. COVID-19 as a subject infects the blood-brain-barrier's endothelial cells and the bloodcerebrospinal fluid barrier's epithelial cells in the choroid plexus brain's ventricles, or leukocytes that become hematogenous propagation vectors [58].

The critical role of proteins $\mathrm{S}$ and $\mathrm{E}$ in $\mathrm{HCoVs}$, specifically $0 \mathrm{C} 43$, and the slow movement of the blood in the brain's microcirculation, can aid in the interaction of the SARS-CoV-2 S protein with the ACE2 receptor expressed in the capillary endothelium. Viral damage and recruitment of endothelial cells can promote invasion of the CNS by SARSCoV-2 [23, 59-62].

In the retrograde neuronal route, infection occurs in the body's periphery and axonal transport mechanisms are used to access the CNS [63, 64]. Respiratory viruses use the olfactory, trigeminal, and vagus cranial nerves to access the brain [65-72].

The four endemic $\mathrm{HCoVs}$ have associations with extra-respiratory diseases such as myocarditis, meningitis, severe diarrhea, and multi-organ failure [73-78]. There have been reports linking the presence of HCoVs in the human CNS and neuro- logical disorders [79-83]. Identifying the etiology of CNS infections poses a challenge. These difficulties arise due to the spectrum of symptoms that infected patients present and the number of pathogens that can cause them. It is also challenging to differentiate a non-viral etiology from systemic viral infection's symptoms outside of the CNS [84-87].

CNS infections can present as meningitis with fever, neck stiffness, photophobia, and phonophobia. Encephalitis' symptoms may be mild and undiagnosed or attributed to other system's imbalances. Typical encephalitic symptoms such as confusion, altered mental status, personality change, abnormal behavior, movement disorders, and seizures have also been reported [84].

In a case series, in which autopsies were performed and brain specimens collected, histopathological studies showed only hypoxic changes without evidence of viral encephalitis in SARS-CoV-2- exposed patients [88]. However, in a study that involved 153 hospitalized patients, clinical data were available for only 125 , which showed that the most common presenting neurological or psychiatric disorder was a cerebrovascular episode in $62 \%$ of patients, followed by altered mental status in $31 \%$ of patients. The altered mental status' etiology, ranged from encephalopathy (nine patients) to encephalitis (seven patients). The remaining 59\% exhibited psychiatric conditions, such as neurocognitive syndrome, psychosis, and affective disorders. Only two patients had exacerbations of pre-existing mental disorders. About $50 \%$ of patients with altered mental status were younger than 60 years of age, while $82 \%$ of patients with cerebrovascular episodes were over 60 years of age [89].

In 214 hospitalized COVID-19 patients in Wuhan, $36.4 \%$ of patients with mild disease had neurological symptoms, and $45.5 \%$ presented them, when severely compromised [90]. Symptoms exhibited are seen in Table 3.

A review of literature has shown COVID-19 to be linked with a high number of cases of ageusia and anosmia. It is due to the entry of the COVID-19 virus through the nasal epithelium, which includes respiratory epithelium and olfactory epithelium [91].

Many viruses, including coronaviruses, do induce brief fluctuations in odor acuity due to different inflammatory responses, including cytokine release. Other $\beta$-coronaviruses often lead to inflammation, while SARS-CoV-2 does not cause nasal epithelium inflammation. The anosmia caused due to SARSCov-2 usually takes weeks for recovery. In contrast, 
Table 3

Neurological Symptoms of COVID-19 [90]

\begin{tabular}{lcllc}
\hline \multicolumn{1}{c}{$\mathrm{CNS}=24.8 \%$} & \multicolumn{1}{c}{ PNS = 8.9\% } & \multicolumn{1}{c}{ Skeletal muscle } \\
\hline Dizziness & $16.80 \%$ & Smell impairment & $5.6 \%$ & Skeletal muscle injury \\
Headache & $13.1 \%$ & Taste impairment & $5.1 \%$ & \\
Altered Consciousness & $7.5 \%$ & Nerve pain & $2.3 \%$ & \\
Acute cerebrovascular disease & $2.8 \%$ & Vision impairment & $1.4 \%$ & \\
Ataxia & $5.0 \%$ & & & \\
Seizures & $5.0 \%$ & & \\
\hline
\end{tabular}

CNS, central nervous system; PNS, peripheral nervous system.

conventional post-viral anosmia is linked to the immediate destruction of olfactory sensory neurons and can take several months for recovery [91].

A study has shown that the identification of nonneuronal cell types in the olfactory epithelium makes patients affected with SARS-CoV-2 highly vulnerable and cause anosmia [91].

Anosmia and ageusia are associated with COVID19 [92, 107]. In a study of 417 European patients, $357(85.6 \%)$ presented viral-associated olfactory dysfunction. $79.6 \%$ presented anosmia, while $20.4 \%$ presented hyposmia. Phantosmia and parosmia represented $12.6 \%$ and $32.4 \%$, respectively. Most (65.4\%) presented olfactory dysfunction after the onset of otorhinolaryngologic symptoms, $22.8 \%$ presented both at the same time, while only $11.8 \%$ before symptom onset. $63 \%$ of all were asymptomatic, but presented olfactory dysfunction persistence [92].

In a French study of 58 COVID-19 patients with ARDS, neurologic features were observed in 49 patients, including prominent agitation, confusion, and corticospinal tract signs [93]. COVID-19 positive patients with strokes have been reported worldwide, and numbers are increasing [94, 98-101]. The virus has not been detected in the cerebrospinal fluid, despite being positive on a COVID-19 nasal swab via RT-PCR. SARS-CoV-2's isolation in the cerebrospinal fluid may be dependent on the disease being systemic or not [102-105]. Further studies will be required to determine if the neurological symptoms are caused by the virus itself or indirectly by secondary, infectious, systemic inflammatory responses. Magnetic resonance imaging (MRI) in the early phase of infection in patients with anosmia, cerebrospinal fluid testing and pathology studies would be helpful to document objective radiological data [106].

While most neurological manifestations have been non-focal, some cases of severe and critical COVID19 have been shown to present with strokes [93].
As per Mao et al., the rate of neurovascular events in their series was about $5.7 \%$ of which about $4.9 \%$ had ischemic strokes [88]. Most ischemic strokes in these case series were subcortical or distal emboli. Some cases were related to other risk factors like atrial fibrillation, diabetes, or hypertension. Other cases, coagulation pathway disorders directly associated with the viral inflammation or immunological response, like antiphospholipid antibodies or elevation of D-dimer and Fibrinogen, may have been responsible [93].

No specific correlation with COVID-19 associated strokes and age or pre-stroke severity of the disease has been found [94]. All the cases had a high neutrophil-lymphocyte ratio except for one. Almost half of the patients who were part of this cohort presented with large vessel occlusions. Eighty percent of those underwent a thrombectomy, most of them having poor outcomes, and this was typically secondary to non-neurological aspects of the disease such as ARDS or multiorgan failure [94]. COVID-19 related ischemic events can follow multiple etiopathogeneses. Hypertension, however, has not been independently associated with severity of COVID-19 [95]. Finally, paradoxical emboli from venous thromboembolism are yet another confounding cause of ischemic events in the brain $[96,97]$.

\section{DIAGNOSIS}

Laboratory diagnostic methods have proven to be reliable in diagnosing COVID-19 and have been presented in tabular form in Table 4 [48, 108].

\section{TREATMENT}

The primary treatment for COVID-19 is symptomatic, if mild. The universal treatment used in patients with moderate, severe, or critical presenta- 
Table 4

Laboratory, Imaging, RT-PCR, and Antigen test common and severe findings. Adapted from [2, 48, 108, 109, 113-116, 118]

\begin{tabular}{|c|c|c|}
\hline & $\begin{array}{l}\text { Early stage of infection/Common } \\
\text { findings }\end{array}$ & Late stage/severe cases of infection/severe findings \\
\hline $\begin{array}{l}\text { Laboratory } \\
\text { findings }\end{array}$ & $\begin{array}{l}\text { Lymphopenia, elevated AST, LDH, muscle enzymes } \\
\text { [108], CRP, and ESR [48]. Procalcitonin values are } \\
\text { normal [48, 109]. }\end{array}$ & $\begin{array}{l}\text { Elevated D-dimer, reduced lymphocyte counts with } \\
\text { altered laboratory values indicating multi-organ failure. } \\
\text { High levels of ALT and AST [48, 108, 109, 113]. IL-6 } \\
\text { induces gene expression and the release of CRP [114]. } \\
\text { IL-6 is part of the host defense mechanism to combat } \\
\text { infections and tissue injuries. When expressed } \\
\text { excessively while fighting SARS-CoV-2, it can trigger a } \\
\text { severe acute systemic inflammatory response, cytokine } \\
\text { storm [115]. Studies have shown that serum levels of } \\
\text { IL-6 and CRP can evaluate severity of disease, predict } \\
\text { the need for mechanical ventilation and estimate } \\
\text { prognosis in COVID-19 patients [114-116]. }\end{array}$ \\
\hline $\begin{array}{l}\text { Imaging } \\
\text { studies }\end{array}$ & $\begin{array}{l}\text { Bilateral, diffuse, patchy ground-glass opacities } \\
\text { with or without consolidations in a peripheral, } \\
\text { posterior, or lower lung zone distribution }[108,109] . \\
\text { Studies revealed that a slight majority of patients } \\
\text { had a negative CT during the first two days of } \\
\text { symptom onset, with a ground-glass opacity usually } \\
\text { developing between day } 0 \text { and } 4 \text { after the beginning } \\
\text { of symptoms, and peaking between day } 6-13 \text { [110]. }\end{array}$ & $\begin{array}{l}\text { Lymphadenopathy and pleural effusion have been } \\
\text { reported more rarely. Other studies report that the } \\
\text { sensitivity of chest CT to reflect SARS-CoV-2 infection } \\
\text { in suspected patients accurately, was } 97 \% \text { based on } \\
\text { RT-PCR positivity, and } 75 \% \text { based on negative RT-PCR } \\
\text { results. These findings showed that chest CT is a } \\
\text { sensitive test to confirm SARS-CoV-2 infection in } \\
\text { conjunction with RT-PCR [2]. }\end{array}$ \\
\hline
\end{tabular}

A negative CT should not be used to exclude the possibility of COVID-19 infection in its early stages. On the contrary, the use of $\mathrm{CT}$ is recommended in clinical management and screening of incidental findings that are potentially attributable to COVID-19 [110]. Available data regarding these CT findings are limited, such that new patterns may emerge with further studies [112]. Some studies suggest that over $20 \%$ of patients with COVID-19 may have coexistent infections, which can obscure the relationship of CT findings to the disease process [110].

\begin{tabular}{|c|c|c|}
\hline RT-PCR & $\begin{array}{l}\text { Main clinical laboratory diagnostic test in } \\
\text { COVID-19 detection [2]. RT-PCR from a respiratory } \\
\text { specimen is currently the most accurate test to } \\
\text { screen and diagnose an active COVID-19 infection. } \\
\text { Not all tests are equivalent, however, and as such } \\
\text { their acceptance on approved test lists is critically } \\
\text { important [110]. The CDC recommends the } \\
\text { collection and testing of an upper respiratory } \\
\text { specimen, with nasopharyngeal being the preferred } \\
\text { route. An oropharyngeal sample, nasal } \\
\text { mid-turbinate swab, and an anterior nares specimen } \\
\text { are acceptable alternatives when the sampling of a } \\
\text { nasopharyngeal swab is not possible. }\end{array}$ & $\begin{array}{l}\text { For patients receiving invasive mechanical ventilation, a } \\
\text { lower respiratory tract aspirate or bronco-alveolar } \\
\text { lavage sample should be collected and tested [112]. The } \\
\text { sample requires storage at } 2-8^{\circ} \mathrm{C} \text { for up to } 72 \mathrm{~h} \text { after } \\
\text { collection. If testing or shipping is delayed, the } \\
\text { specimen should be stored at }-70^{\circ} \mathrm{C} \text { or lower }[112,117] \text {. } \\
\text { The positive rate of RT-PCR for throat swab samples in } \\
\text { early stages of COVID- } 19 \text { has been unacceptably low at } \\
60 \% \text { [118]. In a study of } 51 \text { patients, the positivity rate } \\
\text { for a single respiratory swab was still just } 70 \% \text {, but } \\
\text { adding a second test elevates this to } 94 \% \text {, and a third } \\
\text { test increasing that to } 98 \% \text { [118]. Thus, at least two and } \\
\text { if possible three, independent RT-PCR analyses are } \\
\text { recommended to confirm COVID-19 diagnosis. }\end{array}$ \\
\hline $\begin{array}{l}\text { Antigen } \\
\text { testing }\end{array}$ & \multicolumn{2}{|c|}{$\begin{array}{l}\text { COVID-19 IgM/IgG rapid testing using blood samples revealed a reduced sensitivity in detecting infection, } \\
\text { and often less than } 20 \% \text {. The majority of patients testing positive for COVID-19 by RT-PCR would have } \\
\text { tested negative using the rapid serological assay alone, highlighting the potential for misdiagnosis of } \\
\text { COVID-19 using the latter assay in isolation }[110,111] \text {. The rapid antigen tests are thus not recommended } \\
\text { for screening active COVID-19 infections but rather for monitoring those exposed to COVID-19 previously, } \\
\text { and regardless of recovery. }\end{array}$} \\
\hline
\end{tabular}
ALT, Alanine aminotransferase; AST, Aspartate aminotransferase; CRP, C-Reactive protein; CT, computed tomography; ESR, Erythrocyte sedimentation rate; LDH, lactate dehydrogenase; RT-PCR, Reverse transcription-polymerase chain reaction.

tions is supportive care. In patients presenting with hypoxia or respiratory failure, despite supplemental oxygenation and worsening dyspnea, the use of mechanical ventilation should be considered. Extracorporeal membrane oxygenation should be used as a last resort [2, 7, 14], identifying specific clinical characteristics of critical COVID-19 patients may improve outcome [119].

\section{Mechanical ventilation}

Mechanical ventilation is recommended with lower than normal tidal volumes (4 to $6 \mathrm{ml} / \mathrm{kg}$ predicted body weight) and lower inspiratory pressures, reaching a plateau pressure $<28$ to $30 \mathrm{~cm} \mathrm{H}_{2} \mathrm{O}$. The positive end-expiratory pressure (PEEP) must be high to maintain the driving pressure (Pplat-PEEP) 
low $<14 \mathrm{~cm} \mathrm{H}_{2} \mathrm{O}$ [48]. Avoiding ventilator disconnections is critical to prevent atelectasis and loss of PEEP. Paralytics inducers should be used only if $\mathrm{PaO} 2 / \mathrm{FiO} 2<150 \mathrm{mmHg}$. Prone ventilation lasting longer than 12 hours a day, and conservative fluid management (ARDS strategy in patients without hypoperfusion) are strongly recommended [48, 123].

\section{Antivirals}

Oseltamivir (75 mg BID every 12 hours PO, orally), Ganciclovir (250 mg BID every 12 hours IV, intravenously) ribavirin, Lopinavir/Ritonavir tablets (400/100 mg BID every 12 hours PO, orally) [48] have all been used in efforts to decrease viral load, and to avoid the probability of respiratory complications in several studies [2, 7, 12-14].

Remdesivir, a broad spectrum antiviral [124] that inhibits RNA dependent RNA- polymerase, was successful in the control of in vitro SARS-CoV-2 [12, $125,126]$, and it is hypothesized that it could be efficacious for prophylaxis and therapy of human coronavirus infections [48]. The first dosage under investigation for treatment of COVID-19 was $200 \mathrm{mg}$ intravenously (IV) on day 1 followed by $100 \mathrm{mg}$ IV daily for up to 10 days, infused over 30-60 minutes [127]. More recent studies have demonstrated there is no clinical difference between the administration of the same dosage for 5 or 10 days [128].

\section{Steroids}

The WHO report results from a clinical trial in the United Kingdom where the use of dexamethasone showed promising, lifesaving results for critically ill COVID-19 patients. In the study, preliminary findings showed that mortality was reduced by one-third for patients on ventilators, and one-fifth for patients on oxygen [120].

\section{Anticoagulants}

COVID-19 can induce an immune-thrombotic and disseminated intravascular coagulation, which can explain for thrombosis on a consumptive basis [121]; however, the thrombotic effects of COVID-19 are not completely understood [122].

A non-randomized retrospective study of 351 patients evaluated preemptive administration of prophylactic anticoagulants versus therapeutic anticoagulants. The average age of the participants in the study was 64.7 years old, with a distribution of $58.6 \%$ male and $41.4 \%$ female. A $93.5 \%$ of the sample group were on enoxaparin, $14.8 \%$ on heparin, and some were on both medications. The results showed a difference in in-hospital mortality among COVID-19 patients and those with significantly elevated C-Reactive protein (CRP) levels (>200 mg/L) [122]. The most common causes of death between patients were refractory acute respiratory failure with hypoxia, shock, and multi-organ system failure. Thrombosis could point the etiology of death, but it could also be attributed to direct viral end-organ damage or to a viral systemic inflammatory response syndrome. The study showed no prevention in the progression of disease with a therapeutic dosage of anticoagulation [122].

The risk of mortality was 2.3-fold higher for patients on therapeutic anticoagulants compared to the ones just taking them prophylactically, after controlling other variables. Even for patients with elevated CRP levels, there was no clinical improvement in administering preemptive therapeutic anticoagulation. The study recommends considering the risks and benefits for the patient, as well as a cautious approach in the use of anticoagulation in the management of COVID-19 patients who require them [122].

\section{Convalescent plasma}

In an uncontrolled study of 5 critically ill patients with COVID-19, who were receiving mechanical ventilation, antivirals, and methylprednisolone with an age range of 36-65 years old, the use of convalescent plasma containing neutralizing antibody resulted in clinical improvement of symptoms, ARDS resolution, and weaning of mechanical ventilation, with an increase in the levels of SARS-CoV-2 antibodies [129].

\section{Monoclonal antibodies}

Specific neutralizing monoclonal antibodies, either against receptor-binding domain of spike protein or those binding to ACE2, could effectively block SARS-CoV-2 virus entry. Several monoclonal antibodies have yielded promising results in neutralizing SARS-CoV and MERS-CoV in vitro or in vivo [130].

\section{Anti-malarial}

The combination of Hydroxychloroquine (HCQ) $(600 \mathrm{mg} /$ day $) /$ Azithromycin $(500 \mathrm{mg} /$ day $)$ has been 
used in the treatment against SARS-CoV-2's COVID19 [131]. Both Chloroquine (CQ) and HCQ are weak bases that are known to elevate the $\mathrm{pH}$ of acidic intracellular organelles, such as endosomes and lysosomes that are essential for membrane fusion. It was hypothesized that CQ could inhibit SARS-CoV entry by changing the glycosylation of the ACE2 receptor and spike protein [132].

More recently, a multinational observational, realworld study of hospitalized patients with COVID-19 showed that the use of a regimen containing HCQ or CQ with or without a macrolide was linked with no benefit, but instead associated with an increase in the risk of ventricular arrhythmias and a higher risk for in-hospital death with COVID-19 [133]; however, this study was retracted by the journal that published it, because there were concerns regarding the veracity of the data and analyses conducted by the authors and the database's rights holding corporation [134].

A multi-hospital, retrospective cohort study evaluating clinical outcomes at the Henry Ford Health System in Michigan found that when controlling for COVID-19 risk factors, the treatment with HCQ or HCQ with azithromycin, was associated with reduction of mortality in COVID-19 patients [135]. So far, the information regarding these drugs has been mixed and inconclusive.

The potential treatment for COVID-19/SARSCOV-2 and the mechanisms of action of the various medications at the cellular level including Fusion and endocytosis, translation, proteolysis, RNA Replication to Packaging can be seen as illustrated in Fig. 5.

\section{Vaccines: Traditional protein targets}

There is no FDA approved COVID-19 vaccine available to date. Even with the use of novel platforms, developing a vaccine for SARS-CoV-2 poses a challenge. The optimum design of the immunogen based on viral protein $\mathrm{S}$, the existing adverse effects in vaccines exacerbating lung disease and the uncertainty of whether these new single-dose vaccines will confer immunity and for how long, have yet to be established. Vaccine development is a lengthy and costly process that commonly involves several vaccine candidates and years for approval [48, 136, 145, 147].

The development of a protein subunit vaccine for COVID-19 using microneedle array to deliver MERS-S1 subunits to induce long-lasting potent immunogenicity is underway. Past studies on adenoviral vaccines expressing SARS-CoV- 1 and MERS-S1 subunits demonstrated a more robust antibody-antigen neutralizing activity than when using "S1" alone [136].

The uncertain status of a SARS-CoV-2 vaccine has promoted interest in natural herd immunity to the virus. Herd immunity is the state when a large enough proportion of a population has achieved protective immunity against a pathogen to limit its spread among non-immune individuals critically. It requires protective immunity in $50-90 \%$ of the population, and may be acquired through natural exposure, hence many dangerous pathogens require vaccination to achieve and sustain it [137]. An additional concern with SARS-CoV-2, is that natural exposure may not confer protective immunity to all individuals, as reinfection after initial exposure and evidence of immunosuppression have both been reported [7, 138, 139]. In the case of SARS-CoV-2, it cannot be naturally achieved without dire public health consequences. Assuming protective immunity is required in just $70 \%$ of the US population $(328 \mathrm{M})$, and given infection rates as high as $10-20 \%$, the unrealistic development of immunity in $100 \%$ of those infected [140, 141 ], and mortality as low as $1 \%$, a minimum of 300,000-500,000 COVID-19 deaths can be expected over 7.5-13 months before the US achieved natural herd immunity [142, 143]. While these views are popular, they clash with current knowledge of SARS-CoV-2 infection, mortality, and immune dynamics [144].

The current vaccine development techniques for COVID-19 can be seen in Table 5. Table 6 present a list of vaccines that have been approved for clinical testing along with the different phases they are in currently. Table 7 presents a list of treatment options in development for COVID-19.

\section{Natural killer cells and stem cell therapy}

Natural killer (NK) cells are part of the human innate immunologic system that destroys the virally infected or neoplastic cells. COVID-19 infected patients present a significant decrease of total lymphocytes, CD4-T/CD8-T cells or B cells and NK cells [146].

The purpose of the therapy with NK cells infusion is to benefit those patients with new onset of symptoms or those who have a high risk for severe disease, due to cytokine storm [148]. The infusion of NK cells, derived from placental stem cells, boosts the immune 


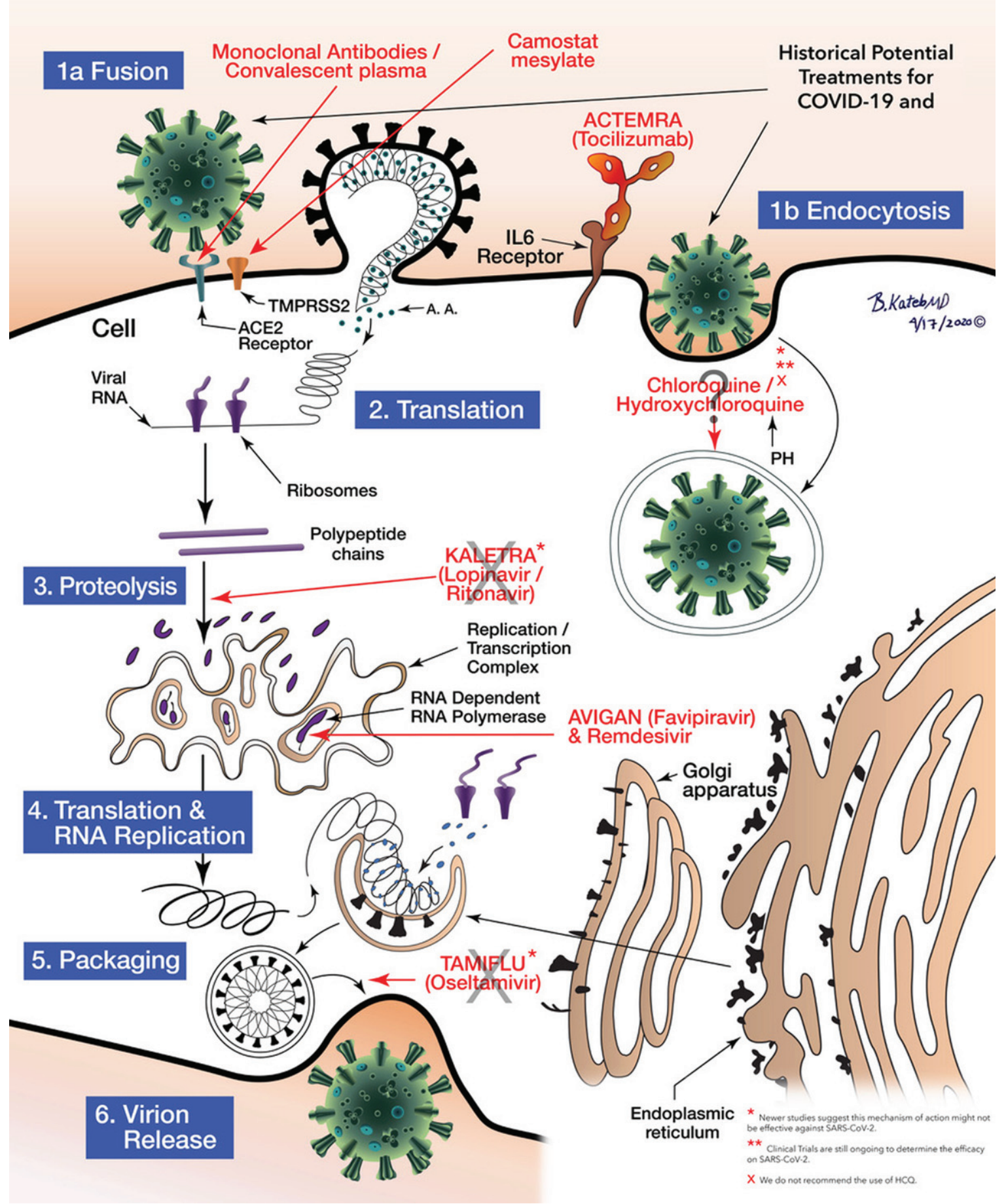

Fig. 5. Schematic illustration of anti COVID-19 medications' mechanisms of action. Illustrated by Dr. Babak Kateb.

response against COVID-19 infected cells, limiting SARS-CoV-2 replication and disease progression by eliminating the infected cells. This option is in the clinical trial phase [148-150].
Mesenchymal stem cells (MSCs) are being used in clinical trials. In one of the studies, two days post-transplantation of MSCs, there was a noticeable improvement of pulmonary functions and symptoms. 
Table 5

COVID-19 Current Vaccine Development Techniques. Adapted from [145]

\begin{tabular}{|c|c|c|c|c|c|c|c|}
\hline \multicolumn{7}{|c|}{ COVID-19 Vaccines Techniques } & \\
\hline \multirow{15}{*}{$\begin{array}{l}\text { Attenuation } \\
\text { Codagenix/ } \\
\text { SII }\end{array}$} & \multirow{2}{*}{$\begin{array}{l}\text { Inactivation } \\
\text { SinoVac/ } \\
\text { Dynavax }\end{array}$} & \multicolumn{2}{|l|}{ Fractionation } & \multicolumn{3}{|l|}{ Cloning } & \\
\hline & & \multicolumn{2}{|c|}{$\begin{array}{l}\text { Non-recombinant purified } \\
\text { purified subunit }\end{array}$} & \multicolumn{2}{|l|}{ Cloning } & \multicolumn{2}{|l|}{ Expression } \\
\hline & & & & $\begin{array}{l}\text { Replication } \\
\text { competent } \\
\text { virus vector } \\
\text { vaccine }\end{array}$ & DNA Vaccine & Protein & \\
\hline & & $\begin{array}{l}\text { Clover Bio- } \\
\text { pharm/GSK }\end{array}$ & Novavax & $\begin{array}{l}\text { Tonix } \\
\text { Pharma/Southern } \\
\text { Research }\end{array}$ & Inovio/Beijing & $\begin{array}{l}\text { Virus-Like } \\
\text { Particle } \\
\text { vaccine }\end{array}$ & $\begin{array}{l}\text { Subunit } \\
\text { Vaccine }\end{array}$ \\
\hline & & $\begin{array}{l}\text { Baylor/UTMB/ } \\
\text { NYBC/Fudan }\end{array}$ & Vaxart & Altimmune & Advaccine & $\begin{array}{l}\text { Geovax } \\
\text { labs/Bravovax }\end{array}$ & $\begin{array}{l}\text { Clover } \\
\text { Biopharm/ } \\
\text { GSK }\end{array}$ \\
\hline & & $\begin{array}{l}\text { UQ/GSK/ } \\
\text { Dynavax }\end{array}$ & Generex & Greffex & Biotech & Medicago & $\begin{array}{l}\text { Baylor/UTMB/ } \\
\text { NYBC/Fudan }\end{array}$ \\
\hline & & $\begin{array}{l}\text { Generex/ } \\
\text { EpiVax }\end{array}$ & ExpreS2ion & Vaxart & $\begin{array}{l}\text { Applied DNA } \\
\text { Sciences }\end{array}$ & & $\begin{array}{l}\text { UQ/GSK/ } \\
\text { Dynavax }\end{array}$ \\
\hline & & Sanofi Pasteur & Vaxil Bio & Zydus & $\begin{array}{l}\text { Takis } \\
\text { Biotech/Evvivax }\end{array}$ & & $\begin{array}{l}\text { Generex/ } \\
\text { EpiVax }\end{array}$ \\
\hline & & $\begin{array}{l}\text { Walter Reed/ } \\
\text { USAMRIID }\end{array}$ & $\begin{array}{l}\text { EpiVax/ } \\
\text { Ugeorgia }\end{array}$ & Cadla & Zydus Cadila & & Sanofi Pasteur \\
\hline & & $\begin{array}{l}\text { iBio/CC- } \\
\text { Pharming }\end{array}$ & & $\begin{array}{l}\text { Institute } \\
\text { Pasteur }\end{array}$ & & & $\begin{array}{l}\text { Walter Reed/ } \\
\text { USAMRIID }\end{array}$ \\
\hline & & & & $\begin{array}{l}\text { Non- } \\
\text { replicating } \\
\text { viral vectors }\end{array}$ & RNA Vaccine & & $\begin{array}{l}\text { iBio/CC- } \\
\text { Pharming }\end{array}$ \\
\hline & & & & $\begin{array}{l}\text { CanSino } \\
\text { (Ad5) }\end{array}$ & Moderna/NIAID & & Novavax \\
\hline & & & & $\begin{array}{l}\text { J\&J/BARDA } \\
(\text { Ad26) }\end{array}$ & CureVac & & Vaxart \\
\hline & & & & $\begin{array}{l}\text { Univ. Oxford } \\
\text { (ChAdOx1) }\end{array}$ & Arcturus/Duke/ & & Generex \\
\hline & & & & & BioNTech/Pfizer & & $\begin{array}{l}\text { ExpreS2ion } \\
\text { Vaxil Bio } \\
\text { EpiVax/ } \\
\text { Ugeorgia }\end{array}$ \\
\hline
\end{tabular}

The laboratory findings showed an increase in peripheral lymphocytes, a decrease in CRP, the complete resolution of the overactivated cytokine secreting immune cells like CD4 + T-cells, CD8 + T-cells, and NK cells, a reduction in TNF- $\alpha$, and an increase in IL-10.

The intravenous infusion of MSCs looks to be a safe alternative for treating patients with severe cases of COVID-19. Despite this data, further studies in a more significant population cohort are needed to corroborate this therapeutic intervention [151].

A study demonstrated the presence of S- reactive CD4 + T cells in COVID-19 patients and also in unexposed healthy individuals. These pre-existing S-reactive T-cells could be cross-reactive clones obtained in prior infections with endemic $\mathrm{HCoVs}$ that account for $\sim 20 \%$ of the yearly common cold.

The function of the pre-existing S-cross-reactive $\mathrm{CD} 4+\mathrm{T}$-cells in $35 \%$ of the healthy individuals is still unknown; however, if these cells are protective in a SARS-CoV-2 infection, they could provide some understanding in the different manifestations of COVID-19, and shed some light in why children and young adults appear to be more resistant to symptomatic SARS-CoV-2 infection [152].

\section{Allogeneic cardiosphere-derived cells}

In a series of non-randomized cases of 6 COVID19 positive patients, with an age range of 19-75 years, cardiosphere-derived cells (CDCs) were used to treat patients who were positive for and exhibited lung injury. CDC's mechanism of action is to target several cytokine pathways (e.g., IL-1, IL-6, $\mathrm{TNF} \alpha$ ), which levels decreased after the infusion. All patients showed clinical recovery and were extubated [153]. 
Table 6

Ongoing clinical trials for COVID-19 vaccines [212-239]

\begin{tabular}{|c|c|c|c|c|c|c|}
\hline Vaccine & Company & Platform & Phase/Status & $\begin{array}{l}\text { Primary } \\
\text { purpose }\end{array}$ & Trial description & Location \\
\hline BCG Vaccine [212] & Texas A\&M University & $\begin{array}{l}\text { Live } \\
\text { attenuated }\end{array}$ & IV Recruiting & Prevention & $\begin{array}{l}\text { A randomized, parallel assignment trial } \\
\mathrm{N}=1800 \text {, health care workers (HCW) } \\
\text { Study start date: April } 20,2020\end{array}$ & USA \\
\hline BCG Vaccine [213] & $\begin{array}{l}\text { Hellenic Institute for the } \\
\text { Study of Sepsis }\end{array}$ & $\begin{array}{l}\text { Live } \\
\text { attenuated }\end{array}$ & IV Recruiting & Prevention & $\begin{array}{l}\text { A randomized, parallel assignment trial } \\
N=900 \\
\text { Study start date: May } 26,2020\end{array}$ & Greece \\
\hline BCG Vaccine [214] & $\begin{array}{l}\text { Murdoch Children's } \\
\text { Research Institute }\end{array}$ & $\begin{array}{l}\text { Live } \\
\text { attenuated }\end{array}$ & III Recruiting & Prevention & $\begin{array}{l}\text { A randomized, parallel assignment trial } \\
\mathrm{N}=4170, \mathrm{HCW} \\
\text { Study start date: March } 30,2020\end{array}$ & Australia \\
\hline BCG Vaccine [215] & UMC Utrecht & $\begin{array}{l}\text { Live } \\
\text { attenuated }\end{array}$ & III Recruiting & Prevention & $\begin{array}{l}\text { A randomized, parallel assignment trial } \\
\mathrm{N}=1500, \mathrm{HCW} \\
\text { Study start date: March } 25,2020\end{array}$ & Netherlands \\
\hline BCG Vaccine [216] & TASK Applied Science & $\begin{array}{l}\text { Live } \\
\text { attenuated }\end{array}$ & III Recruiting & Prevention & $\begin{array}{l}\text { A randomized, parallel assignment trial } \\
\mathrm{N}=500, \mathrm{HCW} \\
\text { Study start date: April } 28,2020\end{array}$ & South Africa \\
\hline VPM1002 [217] & $\begin{array}{l}\text { Vakzine Projekt } \\
\text { Management GmbH }\end{array}$ & $\begin{array}{l}\text { Live } \\
\text { attenuated }\end{array}$ & III Recruiting & Prevention & $\begin{array}{l}\text { A randomized, parallel assignment trial } \\
\mathrm{N}=1200 \text {, HCW } \\
\text { Study start date: May } 25,2020\end{array}$ & Germany \\
\hline VPM1002 [218] & $\begin{array}{l}\text { Vakzine Projekt } \\
\text { Management GmbH }\end{array}$ & $\begin{array}{l}\text { Live } \\
\text { attenuated }\end{array}$ & III Recruiting & Prevention & $\begin{array}{l}\text { A randomized, parallel assignment trial } \\
\mathrm{N}=2038 \text {, people over } 60 \mathrm{y} / \mathrm{o} \\
\text { Estimated study start date: June, } 2020\end{array}$ & Germany \\
\hline MMR vaccine [219] & Kasr El Aini Hospital & $\begin{array}{l}\text { Live } \\
\text { attenuated }\end{array}$ & III Recruiting & Prevention & $\begin{array}{l}\text { A randomized, parallel assignment trial } \\
\mathrm{N}=200 \text {, } \mathrm{HCW} \\
\text { Study start date: June } 16,2020\end{array}$ & Turkey \\
\hline $\begin{array}{l}\text { ChAdOx1 nCoV-19 } \\
\text { [220] }\end{array}$ & University of Oxford & $\begin{array}{l}\text { Non- } \\
\text { Replicating } \\
\text { Viral Vector }\end{array}$ & $\begin{array}{l}\text { II/III } \\
\text { Recruiting }\end{array}$ & Prevention & $\begin{array}{l}\text { A randomized, sequential assignment trial } \\
\mathrm{N}=10260 \\
\text { Study start date: May } 28,2020\end{array}$ & UK \\
\hline Ad5-nCoV [221] & $\begin{array}{l}\text { Institute of } \\
\text { Biotechnology, Academy } \\
\text { of Military Medical } \\
\text { Sciences, PLA of } \\
\text { China/CanSino Bio. Inc. }\end{array}$ & $\begin{array}{l}\text { Non- } \\
\text { Replicating } \\
\text { Viral Vector }\end{array}$ & $\begin{array}{l}\text { II Active, not } \\
\text { recruiting }\end{array}$ & Prevention & $\begin{array}{l}\text { A randomized, crossover assignment for evaluation of } \\
\text { Ad5-nCov, which encodes for full-length S-protein of } \\
\text { SARS-CoV-2. } \\
\mathrm{N}=500 \\
\text { Study start date: April 12, } 2020\end{array}$ & China \\
\hline mRNA-1273 [222] & ModernaTX, Inc. & RNA & II Recruiting & Prevention & $\begin{array}{l}\text { A non-randomized, sequential assignment trial } \\
\mathrm{N}=600 \\
\text { Study start date: May } 29,2020\end{array}$ & USA \\
\hline $\begin{array}{l}\text { Inactivated } \\
\text { SARS-CoV-2 } \\
\text { Vaccine [223] }\end{array}$ & $\begin{array}{l}\text { Sinovac/Development } \\
\text { Co., Ltd. }\end{array}$ & Inactivated & I/II Recruiting & Prevention & $\begin{array}{l}\text { A randomized, parallel assignment trial for the } \\
\text { prevention of the COVID-19 disease } \\
N=744 \\
\text { Study start date: April } 16,2020\end{array}$ & China \\
\hline
\end{tabular}


Table 6

(Continued)

\begin{tabular}{|c|c|c|c|c|c|c|}
\hline Vaccine & Company & Platform & Phase/Status & $\begin{array}{l}\text { Primary } \\
\text { purpose }\end{array}$ & Trial description & Location \\
\hline $\begin{array}{l}\text { Inactivated } \\
\text { SARS-CoV-2 } \\
\text { vaccine (Vero cells) } \\
{[224]}\end{array}$ & $\begin{array}{l}\text { Beijing Institute of } \\
\text { Biological } \\
\text { Products/Sinopharm }\end{array}$ & Inactivated & I/II Recruiting & Prevention & $\begin{array}{l}\text { A randomized, double-blind trial } \\
\mathrm{N}=21 \\
\text { Study start date: April } 28,2020\end{array}$ & China \\
\hline $\begin{array}{l}\text { Inactivated } \\
\text { SARS-CoV-2 } \\
\text { vaccine [225] }\end{array}$ & $\begin{array}{l}\text { Chinese Academy of } \\
\text { Medical Sciences }\end{array}$ & Inactivated & I/II Recruiting & Prevention & $\begin{array}{l}\text { A randomized, parallel assignment, quadruple trial } \\
\mathrm{N}=942 \\
\text { Study start date: May } 15,2020\end{array}$ & China \\
\hline $\begin{array}{l}\text { Gam-COVID-Vac } \\
{[226]}\end{array}$ & $\begin{array}{l}\text { Gamaleya Research } \\
\text { Institute of Epidemiology } \\
\text { and Microbiology, Health } \\
\text { Ministry of the Russian } \\
\text { Federation }\end{array}$ & $\begin{array}{l}\text { Non- } \\
\text { Replicating } \\
\text { Viral Vector }\end{array}$ & I/II Recruiting & Prevention & $\begin{array}{l}\text { A non-randomized, parallel assignment, quadruple trial } \\
\mathrm{N}=38 \\
\text { Study start date: June } 17,2020\end{array}$ & Russia \\
\hline $\begin{array}{l}\text { Gam-COVID-Vac } \\
\text { Lyo [227] }\end{array}$ & $\begin{array}{l}\text { Gamaleya Research } \\
\text { Institute of Epidemiology } \\
\text { and Microbiology, Health } \\
\text { Ministry of the Russian } \\
\text { Federation }\end{array}$ & $\begin{array}{l}\text { Non- } \\
\text { Replicating } \\
\text { Viral Vector }\end{array}$ & I/II Recruiting & Prevention & $\begin{array}{l}\text { A non-randomized, parallel assignment, } \\
\text { quadruple trial } \\
\mathrm{N}=38 \\
\text { Study start date: May } 15,2020\end{array}$ & Russia \\
\hline GX-19 [228] & Genexine, Inc. & DNA & I/II Recruiting & Prevention & $\begin{array}{l}\text { A randomized, parallel assignment, quadruple masked } \\
\text { trial } \\
\mathrm{N}=190 \\
\text { Study start date: June } 17,2020\end{array}$ & $\begin{array}{l}\text { Republic of } \\
\text { Korea }\end{array}$ \\
\hline $\begin{array}{l}\text { Inactivated Vaccine } \\
\text { [229] }\end{array}$ & $\begin{array}{l}\text { Beijing Institute of } \\
\text { Biological } \\
\text { Products/Sinopharm }\end{array}$ & Inactivated & I/II Recruiting & Prevention & $\begin{array}{l}\text { A randomized, parallel assignment } \\
\text { trial } \\
\mathrm{N}=1448 \\
\text { Study start date: April 11, } 2020\end{array}$ & China \\
\hline INO-4800 [230] & Inovio Pharmaceuticals & DNA & I Recruiting & Prevention & $\begin{array}{l}\text { A non-randomized, sequential assignment trial } \\
\mathrm{N}=40 \\
\text { Study start date: April 3, } 2020\end{array}$ & USA \\
\hline $\begin{array}{l}\text { NVX-CoV2373 } \\
{[231]}\end{array}$ & Novavax & $\begin{array}{l}\text { Protein } \\
\text { Subunit }\end{array}$ & I Recruiting & Prevention & $\begin{array}{l}\text { A randomized, parallel assignment, triple masked } \\
\text { trial } \\
\mathrm{N}=131 \\
\text { Study start date: May } 25,2020\end{array}$ & Australia \\
\hline mRNA-1273 [232] & $\begin{array}{l}\text { National Institute of } \\
\text { Allergy and Infectious } \\
\text { Diseases (NIAID) }\end{array}$ & RNA & I Recruiting & Prevention & $\begin{array}{l}\text { A non-randomized, sequential assignment } \\
\text { trial } \\
\mathrm{N}=155 \\
\text { Study start date: March 16, } 2020\end{array}$ & USA \\
\hline
\end{tabular}




\begin{tabular}{|c|c|c|c|c|c|c|}
\hline CVnCoV [233] & CureVac AG & RNA & I Recruiting & Prevention & $\begin{array}{l}\text { A randomized, sequential assignment, single masked } \\
\text { trial } \\
N=168 \\
\text { Study start date: June } 18,2020\end{array}$ & $\begin{array}{l}\text { Belgium/ } \\
\text { Germany }\end{array}$ \\
\hline SCB-2019 [234] & $\begin{array}{l}\text { Clover } \\
\text { Biopharmaceuticals AUS } \\
\text { Pty Ltd }\end{array}$ & $\begin{array}{l}\text { Protein } \\
\text { Subunit }\end{array}$ & I Recruiting & Prevention & $\begin{array}{l}\text { A randomized, sequential assignment, triple masked } \\
\text { trial } \\
\mathrm{N}=150 \\
\text { Study start date: June } 19,2020\end{array}$ & Australia \\
\hline $\begin{array}{l}\text { COVAX-19 vaccine } \\
{[235]}\end{array}$ & Vaxine Pty Ltd & $\begin{array}{l}\text { Protein } \\
\text { Subunit }\end{array}$ & I Recruiting & Prevention & $\begin{array}{l}\text { A randomized, parallel assignment, triple masked trial } \\
N=40 \\
\text { Study start date: June } 30,2020\end{array}$ & Australia \\
\hline V-SARS [236] & Immunitor LLC & Inactivated & $\begin{array}{l}\text { I/II Active, not } \\
\text { recruiting }\end{array}$ & Treatment & $\begin{array}{l}\text { An interventional, single group assignment for } \\
\text { treatment of COVID-19 with pill } \\
\mathrm{N}=20 \\
\text { Study start date: May } 15,2020\end{array}$ & $\begin{array}{l}\text { Canada/ } \\
\text { Mongolia }\end{array}$ \\
\hline $\begin{array}{l}\text { LV-SMENP-DC } \\
{[237]}\end{array}$ & $\begin{array}{l}\text { Shenzhen Geno-Immune } \\
\text { Medical Institute }\end{array}$ & Lentiviral & I/II Recruiting & Treatment & $\begin{array}{l}\text { An interventional, single group assignment for } \\
\text { treatment of COVID-19 with novel lentiviral-based DC } \\
\text { and T cell vaccines } \\
N=100 \\
\text { Study start date: March } 24,2020\end{array}$ & China \\
\hline BNT162 [238] & BioNTech/ Pfizer & RNA & I/II Ongoing & Treatment & $\begin{array}{l}\text { A non-randomized, sequential assignment trial to test } \\
\text { the effect of four different BNT162 vaccines against } \\
\text { COVID-19 in healthy individuals } \\
N=200 \\
\text { Study start date: April } 23,2020\end{array}$ & Germany \\
\hline aAPC vaccine [239] & $\begin{array}{l}\text { Shenzhen Geno-Immune } \\
\text { Medical Institute }\end{array}$ & Lentiviral & I Recruiting & Treatment & $\begin{array}{l}\text { An open-label, single-group assignment } \\
N=100 \\
\text { Study start date: February } 15,2020\end{array}$ & China \\
\hline
\end{tabular}


Table 7

Ongoing clinical trials for COVID-19 treatments [240-425]

\begin{tabular}{|c|c|c|c|c|c|c|}
\hline Treatment goal & Target & Drug & Company & Stage & Reference & Location \\
\hline Prevention & $\begin{array}{l}\text { Pre-exposure } \\
\text { phrophylaxis }\end{array}$ & Truvada & Gilead & Phase III & [240] & Spain \\
\hline Prevention & Nitric oxide & GLS-1200 & $\begin{array}{l}\text { GeneOne Life } \\
\text { Science }\end{array}$ & Phase II & [241] & USA \\
\hline Prevention & $\begin{array}{l}\text { Broad-spectrum } \\
\text { antiseptic }\end{array}$ & Povidone-Iodine & $\begin{array}{l}\text { University of } \\
\text { Kentucky }\end{array}$ & Phase II & [242] & USA \\
\hline $\begin{array}{l}\text { Prevent respiratory } \\
\text { infection }\end{array}$ & $\begin{array}{l}\text { Pan antiviral } \\
\text { immunotherapy }\end{array}$ & RTB101 & Restorbio & Phase III & [243] & USA \\
\hline $\begin{array}{l}\text { Prevent respiratory } \\
\text { illness }\end{array}$ & Anti-GM-CSF mAb & Otilimab & GSK & Phase II & [244] & USA, Spain \\
\hline $\begin{array}{l}\text { Prevent respiratory } \\
\text { illness }\end{array}$ & Anti-GM-CSF mAb & Mavrilimumab & $\begin{array}{l}\text { Kiniksa } \\
\text { Pharmaceuticals }\end{array}$ & Phase II & {$[245]$} & USA \\
\hline $\begin{array}{l}\text { Prevent respiratory } \\
\text { illness and heart } \\
\text { failure }\end{array}$ & Anti Il-1B mAb & Ilaris & Novartis & Phase II & [246] & USA \\
\hline $\begin{array}{l}\text { Prevent shortness of } \\
\text { breath }\end{array}$ & SSRI & Fluvoxamine & $\begin{array}{l}\text { Washington } \\
\text { University School } \\
\text { of Medicine }\end{array}$ & Phase II & [247] & USA \\
\hline Anti-viral growth & EF1A inhibitor & Aplidin & PharmaMar & Phase I & [248] & Spain \\
\hline Anti-viral growth & Il-29 agonist & $\begin{array}{l}\text { Peginterferon } \\
\text { Lambda-1a }\end{array}$ & Eiger BioPharma & Phase II & [249] & USA \\
\hline Anti-viral growth & Anti-factor XIIa mAb & CSL312 & CSL Behring & Phase II & {$[250]$} & USA \\
\hline Anti-viral growth & Plant-derived antiviral & $\mathrm{AQCH}$ & Sun Pharma & Phase II & [251] & India \\
\hline Anti-viral growth & HIV protease inhibitor & $\begin{array}{l}\text { Kaletra (Lopinavir } \\
\text { - Ritonavir) }\end{array}$ & Abbvie & Failed trial & [252-255] & $\begin{array}{l}\text { Multiple } \\
\text { locations }\end{array}$ \\
\hline Anti-viral growth & $\begin{array}{l}\text { Broad-spectrum } \\
\text { antiviral }\end{array}$ & Arbidol & Pharmstandard & $\begin{array}{l}\text { Failed clinical } \\
\text { trial }\end{array}$ & {$[256]$} & China, Iran \\
\hline Anti-viral growth & $\begin{array}{l}\text { Broad-spectrum } \\
\text { antiviral }\end{array}$ & EIDD-2801 & Ridgeback Bio & Phase I & {$[257]$} & $\begin{array}{l}\text { United } \\
\text { Kingdom }\end{array}$ \\
\hline Anti-viral growth & $\begin{array}{l}\text { Broad-spectrum } \\
\text { antiviral, antiparasitic }\end{array}$ & Alinia & $\begin{array}{l}\text { Romark } \\
\text { Laboratories }\end{array}$ & Phase III & {$[258-261]$} & $\begin{array}{l}\text { Multiple } \\
\text { locations }\end{array}$ \\
\hline Anti-viral growth & $\begin{array}{l}\text { Broad-spectrum } \\
\text { antiparasitic }\end{array}$ & Ivermectin & Tanta University & Phase II/III & {$[258]$} & $\begin{array}{l}\text { Multiple } \\
\text { locations }\end{array}$ \\
\hline Anti-viral growth & Cleaves sialic acid & Fludase (DAS181) & Ansun Biopharma & Phase III & {$[262,263]$} & $\begin{array}{l}\text { Multiple } \\
\text { locations }\end{array}$ \\
\hline Anti-viral growth & Adenosine analog & Remdesivir & Gilead & $\begin{array}{l}\text { Approved/ } \\
\text { EUA/ Phase } \\
\text { III }\end{array}$ & {$[264,265]$} & $\begin{array}{l}\text { Multiple } \\
\text { locations }\end{array}$ \\
\hline Anti-viral growth & Adenosine analog & Galidesivir & $\begin{array}{l}\text { BioCryst } \\
\text { Pharmaceuticals }\end{array}$ & Phase I & [266] & Brazil \\
\hline Anti-viral growth & Guanosine analog & Virazole & Bausch Health & Phase II & [267] & Canada \\
\hline Anti-viral growth & Pyrimidine analog & Clevudine & $\begin{array}{l}\text { Bukwang } \\
\text { Pharmaceutical }\end{array}$ & Phase II & [268] & Korea \\
\hline Anti-viral growth & $\begin{array}{l}\text { RNA polymerase } \\
\text { inhibitor }\end{array}$ & $\begin{array}{l}\text { Avigan } \\
\text { (Favipiravir) }\end{array}$ & Fujifilm & Phase III & [269-272] & $\begin{array}{l}\text { Multiple } \\
\text { locations }\end{array}$ \\
\hline Anti-viral growth & $\begin{array}{l}\text { Non-nucleoside } \\
\text { reverse transcriptase } \\
\text { inhibitor }\end{array}$ & $\begin{array}{l}\text { Elpida } \\
\text { (elsulfavirine) }\end{array}$ & Viriom & Phase II & [273] & $\begin{array}{l}\text { Russian } \\
\text { Federation }\end{array}$ \\
\hline Anti-viral growth & $\begin{array}{l}\text { Nucleoside reverse } \\
\text { transcriptase inhibitor }\end{array}$ & Azvudine & $\begin{array}{l}\text { Capital Medical } \\
\text { University }\end{array}$ & Phase II & [274] & China \\
\hline Anti-viral growth & ACE-2 inhibitor & $\begin{array}{l}\text { Chloroquine/ } \\
\text { Hydroxy- } \\
\text { chloroquine }\end{array}$ & Multiple locations & $\begin{array}{l}\text { Failed / } \\
\text { Ongoing }\end{array}$ & {$[275,276]$} & $\begin{array}{l}\text { Multiple } \\
\text { locations }\end{array}$ \\
\hline Anti-viral growth & VEGF inhibitor & Avastin & Roche & Phase II/III & {$[277,278]$} & China, Italy \\
\hline Anti-viral growth & Inhaled corticosteroid & $\begin{array}{l}\text { Alvesco } \\
\text { (ciclesonide) }\end{array}$ & Covis Pharma & Phase III & [279] & Korea, Japan \\
\hline Anti-viral growth & $\mathrm{H} 2$ antagonist & $\begin{array}{l}\text { Pepcid } \\
\text { (Famotidine) }\end{array}$ & Northwell Health & Phase II & [280] & USA \\
\hline Anti-viral growth & $\begin{array}{l}\text { Anti-Spike (S), } \\
\text { SARS-CoV }-2 \text { mAb }\end{array}$ & $\begin{array}{l}\text { REGN-COV2 } \\
\text { (REGN10933 + } \\
\text { REGN10987) }\end{array}$ & Regeneron & Phase III & {$[281,282]$} & USA \\
\hline
\end{tabular}


Table 7

(Continued)

\begin{tabular}{|c|c|c|c|c|c|c|}
\hline Treatment goal & Target & Drug & Company & Stage & Reference & Location \\
\hline Anti-viral growth & $\begin{array}{l}\text { Anti-SARS-CoV - } 2 \\
\mathrm{mAb}\end{array}$ & JS016 & Junshi Biosciences & Phase I & [283] & China \\
\hline Anti-viral growth & $\begin{array}{l}\text { Anti-SARS-CoV - } 2 \\
\text { antibody }\end{array}$ & LY-CoV555 & Eli Lilly, AbCellera & Phase I & [284] & USA \\
\hline Anti-viral growth & $\begin{array}{l}\text { Anti-SARS-CoV - } 2 \\
\text { antibody }\end{array}$ & TY027 & Tychan & Phase I & [285] & Singapore \\
\hline Anti-viral growth & Ion channel blocker & Amiodarone & $\begin{array}{l}\text { Nicolaus } \\
\text { Copernicus } \\
\text { University }\end{array}$ & Phase II/III & [286] & Poland \\
\hline Anti-viral growth & Ion channel blocker & Verapamil & $\begin{array}{l}\text { Nicolaus } \\
\text { Copernicus } \\
\text { University }\end{array}$ & Phase II/III & [286] & Poland \\
\hline Anti-viral growth & $\begin{array}{l}\text { Oral purine nucleotide } \\
\text { prodrug }\end{array}$ & AT-527 & $\begin{array}{l}\text { Atea } \\
\text { Pharmaceuticals }\end{array}$ & Phase II & [287] & USA \\
\hline Anti-viral growth & IMPDH inhibitor & Vicromax & ViralClear Pharma & Phase II & {$[288]$} & USA \\
\hline Treat hypoxia & HIF-PH inhibitor & Desidustat & Zydus Cadila & Phase II & [289] & Mexico \\
\hline Treat hypertension & Renin inhibitor & Aliskiren & Sichuan University & Phase II & [290] & China \\
\hline Treat pneumonia & Anti-CD6 mAb & Itolizumab & $\begin{array}{l}\text { Center of molecular } \\
\text { immunology }\end{array}$ & Phase II & [291] & Cuba \\
\hline Treat pneumonia & NRP2 modulator & ATYR1923 & aTYR Pharma & Phase II & [292] & USA \\
\hline Treat pneumonia & $\begin{array}{l}\text { CRAC channel } \\
\text { inhibitor }\end{array}$ & CM4620 & CalciMedica & Phase II & [293] & USA \\
\hline Treat pneumonia & $\begin{array}{l}\text { Bacterial mucosa } \\
\text { vaccine }\end{array}$ & Bactek - R & Immunotek & Phase III & [294] & $\begin{array}{l}\text { Dominican } \\
\text { Republic }\end{array}$ \\
\hline Treat pneumonia & $\begin{array}{l}\text { Hep C/HIV protease } \\
\text { inhibitors }\end{array}$ & Ganovo + Ritonavir & Ascletis & Phase IV & [295] & China \\
\hline Treat pneumonia & JAK inhibitor & Jakavi & Novartis, Incyte & Phase III & [296] & $\begin{array}{l}\text { Multiple } \\
\text { locations }\end{array}$ \\
\hline Treat pneumonia & Jak1/3 inhibitor & Xeljanz & Pfizer & Phase II & [297] & Italy \\
\hline Treat pneumonia & Iron chelator & Desferal & $\begin{array}{l}\text { Kermanshan } \\
\text { University of } \\
\text { Medical Sci. }\end{array}$ & Phase I/II & [298] & Iran \\
\hline Treat pneumonia & Anti-CD147 antibody & Meplazumab & Tang-Du Hospital & Phase I/II & [299] & China \\
\hline Treat pneumonia & $\begin{array}{l}\text { HIV - } 1 \text { protease } \\
\text { inhibitor+CYP3A } \\
\text { inhitor }\end{array}$ & Prezcobix & $\begin{array}{l}\text { Shanghai Public } \\
\text { Health Clinical } \\
\text { Center }\end{array}$ & Phase III & {$[300]$} & China \\
\hline Treat pneumonia & Il-33 inhibitor & MSTT1041A & Genentech & Phase II & {$[301]$} & USA \\
\hline Treat pneumonia & Il-22 receptor agonist & UTTR1147A & Genentech & Phase II & [301] & USA \\
\hline Anti-inflammatory & $\begin{array}{l}\text { Complement (C3) } \\
\text { inhibitor }\end{array}$ & APL-9 & Apellis Pharma & Phase I/II & [302] & USA \\
\hline Anti-inflammatory & $\begin{array}{l}\text { Recombinat human } \\
\mathrm{C} 1 \text { inhibitor }\end{array}$ & Ruconest & Pharming & Phase II & {$[303]$} & Switzerland \\
\hline Anti-inflammatory & Anti-GM-CSF mAb & TJ003234 & I-mab Biopharma & Phase I/II & [304] & USA \\
\hline Anti-inflammatory & DPEP-1 inhibitor & MetaBlok & Arch Biopartnes & Phase II & [305] & Canada \\
\hline Anti-inflammatory & $\begin{array}{l}\text { Tyrosine kinase } \\
\text { inhibitor }\end{array}$ & Masitinib & AB Science & Phase II & [306] & France \\
\hline Anti-inflammatory & Antioxidant & $\mathrm{N}$-acetylcysteine & MSKCC & Phase II & {$[307]$} & USA \\
\hline Anti-inflammatory & Antioxidant & Viusid and Asbrip & Catalysis SL & Phase II & [308] & Ecuador \\
\hline Anti-inflammatory & Oral Fenretinide & LAU-7b & $\begin{array}{l}\text { Laurent } \\
\text { Pharmaceuticals }\end{array}$ & Phase II & [309] & Canada \\
\hline Anti-inflammatory & $\begin{array}{l}\text { Alpha- } 1 \text { adrenergic } \\
\text { receptor blocker }\end{array}$ & Prazosin & $\begin{array}{l}\text { Johns Hopkins } \\
\text { University }\end{array}$ & Phase II & [310] & USA \\
\hline Anti-inflammatory & $\begin{array}{l}\text { Inhibits DNA } \\
\text { synthesis }\end{array}$ & Etoposide & $\begin{array}{l}\text { Boston Medical } \\
\text { Center }\end{array}$ & Phase II & [311] & USA \\
\hline Anti-inflammatory & $\begin{array}{l}\text { Angiotensin II } \\
\text { receptor blocker }\end{array}$ & Telmisartan & Laboratorio Elea & Phase II & {$[312]$} & Argentina \\
\hline Anti-inflammatory & $\begin{array}{l}\text { Anti-light (TNFSF } \\
\text { 14) } \mathrm{mAb}\end{array}$ & CERC-002 & Cerecor & Phase II & [313] & USA \\
\hline Anti-inflammatory & AntiTNF antibody & Remsima & Celltrion Healthcare & Phase I & [314] & USA \\
\hline Anti-inflammatory & AT2r Agonist & VP01(C21) & Vicore Pharma & Phase II & [315] & USA \\
\hline
\end{tabular}


Table 7

(Continued)

\begin{tabular}{|c|c|c|c|c|c|c|}
\hline Treatment goal & Target & Drug & Company & Stage & Reference & Location \\
\hline Anti-inflammatory & $\begin{array}{l}\text { Live biotherapeutic } \\
\text { product }\end{array}$ & MRx-4DP0004 & 4D Pharma & Phase II & [316] & $\begin{array}{l}\text { United } \\
\text { Kingdom }\end{array}$ \\
\hline Anti-inflammatory & XPO1 inhibitor & Xpovio & $\begin{array}{l}\text { Karyopharm } \\
\text { Therapeutics }\end{array}$ & Phase II & [317] & USA \\
\hline Anti-inflammatory & Opioid antagonist & Naltrexone & Beaumont Health & Phase II & [318] & USA \\
\hline Anti-inflammatory & NMDA antagonist & Ketamine & Beaumont Health & Phase II & [318] & USA \\
\hline Anti-inflammatory & BTK inhibitor & Brukinsa & BeiGene & Phase II & [319] & USA \\
\hline Anti-inflammatory & BTK inhibitor & Calquence & Astrazeneca & Phase II & {$[320,321]$} & $\begin{array}{l}\text { Multiple } \\
\text { locations }\end{array}$ \\
\hline Anti-inflammatory & HMGB1 inhibitor & DSTAT & Chimerix & Phase II/III & [322] & USA \\
\hline Anti-inflammatory & $\begin{array}{l}\text { Recombinant } \\
\text { humanized GM-CSF }\end{array}$ & Leukine & $\begin{array}{l}\text { Partner } \\
\text { therapeutics }\end{array}$ & Phase IV & [323] & Belgium \\
\hline Anti-inflammatory & $\begin{array}{l}\text { Upregulate RNA } \\
\text { splicing }\end{array}$ & $\mathrm{ABX} 464$ & Abivax & Phase II/III & [324] & France \\
\hline Anti-inflammatory & IL-6 inhibitor $\mathrm{mAb}$ & Clazakizumab & Vitaeris & Phase II & {$[325]$} & USA \\
\hline Anti-inflammatory & Anti- IL-8 mAb & BMS-986253 & $\begin{array}{l}\text { Bristol Myers } \\
\text { Squibb }\end{array}$ & Phase II & [326] & USA \\
\hline Anti-inflammatory & IL-6 inhibitor & Sirukumab & Janssen & Phase II & {$[327]$} & USA \\
\hline Anti-inflammatory & IL-6 inhibitor & Actemra & Roche & Phase III & [328-331] & $\begin{array}{l}\text { Multiple } \\
\text { locations }\end{array}$ \\
\hline Anti-inflammatory & IL-6 inhibitor & $\mathrm{CD} 24 \mathrm{Fc}$ & Oncoimmune & Phase III & [332] & USA \\
\hline Anti-inflammatory & IL-6 inhibitor & Kevzara & Regeneron, Sanofi & Phase II/III & [333] & $\begin{array}{l}\text { Multiple } \\
\text { locations }\end{array}$ \\
\hline Anti-inflammatory & IL-6 inhibitor & Aviptadil & $\begin{array}{l}\text { NeuroRX, relief } \\
\text { therapeutics }\end{array}$ & Phase II & [334] & USA \\
\hline Anti-inflammatory & IL-6 inhibitor & Sylvant & EUSA Pharma & Phase III & {$[335,336]$} & $\begin{array}{l}\text { Multiple } \\
\text { locations }\end{array}$ \\
\hline Anti-inflammatory & IL-6 inhibitor & Levilimab & Biocad & Phase III & {$[337]$} & $\begin{array}{l}\text { Russian } \\
\text { Federation }\end{array}$ \\
\hline Anti-inflammatory & IL-6 inhibitor & Olokizumab & R-Pharm & Phase II/III & [338] & $\begin{array}{l}\text { Russian } \\
\text { Federation }\end{array}$ \\
\hline Anti-inflammatory & Il-7 agonist & CYT107 & RevImmune & Phase II & [339] & $\begin{array}{l}\text { United } \\
\text { Kingdom }\end{array}$ \\
\hline Anti-inflammatory & TLR 7/8 antagonist & M5049 & Merck KGaA & Phase II & [340] & USA \\
\hline Anti-inflammatory & Anti - GM -CSF & Lenzilumab & Humanigen & Phase III & [341] & USA \\
\hline Anti-inflammatory & Anti - GM -CSF & Gimsilumab & $\begin{array}{l}\text { Roivant, } \\
\text { Altasciences }\end{array}$ & Phase II & [342] & USA \\
\hline bAnti-inflammatory & Turbulin disruption & Colchicine & $\begin{array}{l}\text { Montreal Heart } \\
\text { Institute }\end{array}$ & Phase III & [343] & Canada \\
\hline Anti-inflammatory & NK1 inhibitor & Tradipitant & $\begin{array}{l}\text { Vanda } \\
\text { Pharmaceuticals }\end{array}$ & Phase III & [344] & USA \\
\hline Anti-inflammatory & $\mathrm{C} 5 \mathrm{a} \mathrm{mAb}$ & IFX-1 & InflaRX & Phase II/III & {$[345]$} & Netherlands \\
\hline Anti-inflammatory & IL-1 inhibitor & Kineret & Sobi & Phase III & [346] & Italy \\
\hline Anti-inflammatory & IL-1B inhibitor & RPH-104 & R-Pharm & Phase II/III & [338] & Russia \\
\hline Anti-inflammatory & Il-15 superagonist & $\mathrm{N}-803$ & ImmunityBio & Phase I & [347] & USA \\
\hline Anti-inflammatory & IFNy $\mathrm{mAb}$ & Gamifant & Sobi & Phase II/III & [346] & Italy \\
\hline Anti-inflammatory & $\begin{array}{l}\text { CGRP receptor } \\
\text { antagonist }\end{array}$ & Vazegepant & Biohaven Pharma & Phase II/III & [348] & USA \\
\hline Anti-inflammatory & CCR5 antagonist & $\begin{array}{l}\text { Leronlimab (PRO } \\
140)\end{array}$ & CytoDyn & Phase II & [349] & USA \\
\hline Anti-inflammatory & $\begin{array}{l}\text { Sphingosine } \\
\text { 1-phosphate receptor } \\
\text { modulator }\end{array}$ & Gilenya & Novartis & Phase II & {$[350]$} & China \\
\hline Anti-inflammatory & Tissue regeneration & $\begin{array}{l}\text { Mesenchymal } \\
\text { Stem cells }\end{array}$ & $\begin{array}{l}\text { Multiple } \\
\text { companies }\end{array}$ & Phase II & [351-365] & $\begin{array}{l}\text { Multiple } \\
\text { locations }\end{array}$ \\
\hline Anti-inflammatory & $\begin{array}{l}\text { Mesenchymal Stem } \\
\text { cells }\end{array}$ & RYONCIL & Mesoblast & Phase III & {$[366]$} & USA \\
\hline Anti-inflammatory & $\begin{array}{l}\text { A3 adenosine receptor } \\
\text { agonist }\end{array}$ & Piclidenoson & $\begin{array}{l}\text { Can-Fite } \\
\text { Biopharma }\end{array}$ & Phase II & {$[367]$} & Israel \\
\hline Anti-inflammatory & $\begin{array}{l}\text { Jak2, IRAK-1, } \\
\text { CSF1R inhibitors }\end{array}$ & Pacritinib & CTI Biopharma & Phase III & {$[368]$} & USA \\
\hline
\end{tabular}


Table 7

(Continued)

\begin{tabular}{|c|c|c|c|c|c|c|}
\hline Treatment goal & Target & Drug & Company & Stage & Reference & Location \\
\hline Anti-inflammatory & $\begin{array}{l}\text { Suprapharmacologic } \\
\text { dexamethasone }\end{array}$ & AVM0703 & $\begin{array}{l}\text { AVM } \\
\text { Biotechnology }\end{array}$ & Phase I/II & [369] & Denmark \\
\hline $\begin{array}{l}\text { Anti-inflammatory, } \\
\text { treat respiratory } \\
\text { illness }\end{array}$ & Corticosteroid & Dexamethasone & $\begin{array}{l}\text { University of } \\
\text { Oxford }\end{array}$ & Phase II/III & {$[370]$} & Multiple locations \\
\hline Anti-coagulant & Antithrombotic activity & Enoxaparin & NYU Langone & Phase III & [371] & USA \\
\hline $\begin{array}{l}\text { Block viral entry into } \\
\text { cells }\end{array}$ & Jak1/2 inhitor & Olumiant & Eli Lilly & Phase III & {$[372,373]$} & Italy, USA \\
\hline $\begin{array}{l}\text { Block viral entry into } \\
\text { cells }\end{array}$ & AXL Kinase inhibitor & Bemcentinib & BerGenBio & Phase II & [374] & United Kingdom \\
\hline $\begin{array}{l}\text { Block viral entry into } \\
\text { cells }\end{array}$ & $\begin{array}{l}\text { Serine protease } \\
\text { inhibitor }\end{array}$ & Camostat Mesilate & $\begin{array}{l}\text { University of } \\
\text { Aarhus }\end{array}$ & Phase I/II & {$[375]$} & Denmark \\
\hline $\begin{array}{l}\text { Block viral entry into } \\
\text { cells }\end{array}$ & $\begin{array}{l}\text { Serine protease } \\
\text { inhibitor }\end{array}$ & Nafamostat & $\begin{array}{l}\text { University Hospital } \\
\text { Padova }\end{array}$ & Phase II/III & [376] & Italy \\
\hline $\begin{array}{l}\text { Treat respiratory } \\
\text { illness }\end{array}$ & Regenerative medicine & PLX cell therapy & $\begin{array}{l}\text { Pluristem } \\
\text { Therapeutics }\end{array}$ & Phase II & [377] & USA \\
\hline $\begin{array}{l}\text { Treat respiratory } \\
\text { illness }\end{array}$ & $\begin{array}{l}\text { Nebulized pan-Jak } \\
\text { inhibitor }\end{array}$ & TD-0903 & Theravance & Phase I & {$[378]$} & United Kingdom \\
\hline $\begin{array}{l}\text { Treat respiratory } \\
\text { illness }\end{array}$ & NMDA antagonist & LEAF-4L6715 & LEAF 4Life & Phase I/II & [379] & France \\
\hline $\begin{array}{l}\text { Treat respiratory } \\
\text { illness }\end{array}$ & Humanized IgG4 mAb & Axatilimab & $\begin{array}{l}\text { Syndax } \\
\text { Pharmaceuticals }\end{array}$ & Phase II & {$[380]$} & USA \\
\hline $\begin{array}{l}\text { Treat respiratory } \\
\text { illness }\end{array}$ & Alkylating agent & $\begin{array}{l}\text { Melphalan } \\
\text { Inhalation }\end{array}$ & $\begin{array}{l}\text { Fed state Budgetary } \\
\text { Institute }\end{array}$ & Phase II & [381] & $\begin{array}{l}\text { Russian } \\
\text { Federation }\end{array}$ \\
\hline $\begin{array}{l}\text { Treat respiratory } \\
\text { illness }\end{array}$ & Complement inhibitor & Soliris & $\begin{array}{l}\text { Alexion } \\
\text { Pharmaceuticals }\end{array}$ & Phase II & [382] & France \\
\hline $\begin{array}{l}\text { Treat respiratory } \\
\text { illness }\end{array}$ & $\begin{array}{l}\text { Oral selective } \\
\text { antitubulin }\end{array}$ & Veru-111 & Veru & Phase II & [383] & USA \\
\hline $\begin{array}{l}\text { Treat respiratory } \\
\text { illness }\end{array}$ & mTOR inhibitor & Sirolimus & $\begin{array}{l}\text { University of } \\
\text { Cincinnati }\end{array}$ & Phase II & [384] & USA \\
\hline $\begin{array}{l}\text { Treat respiratory } \\
\text { illness }\end{array}$ & NS & DFV890 & Novartis & Phase II & [385] & Denmark \\
\hline $\begin{array}{l}\text { Treat respiratory } \\
\text { illness }\end{array}$ & NS & MAS825 & Novartis & Phase II & [386] & USA \\
\hline $\begin{array}{l}\text { Treat respiratory } \\
\text { illness }\end{array}$ & Anti-c5a antibody & BDB-001 & $\begin{array}{l}\text { Staidson biopharma, } \\
\text { Bejing Defengrui } \\
\text { Biotech }\end{array}$ & Phase II/III & {$[387]$} & China, Indonesia \\
\hline $\begin{array}{l}\text { Treat respiratory } \\
\text { illness }\end{array}$ & Anti-c5aR antibody & Avdoralimab & Innate Pharma & Phase II & [388] & France \\
\hline $\begin{array}{l}\text { Treat respiratory } \\
\text { illness }\end{array}$ & Interferon beta $-1 \mathrm{a}$ & Rebif & $\begin{array}{l}\text { Merck KgaA, } \\
\text { INSERM }\end{array}$ & Phase III & [389] & France \\
\hline $\begin{array}{l}\text { Treat respiratory } \\
\text { illness }\end{array}$ & Interferon beta $-1 \mathrm{a}$ & SNG001 & Synairgen & Phase II & [390] & England \\
\hline $\begin{array}{l}\text { Treat respiratory } \\
\text { illness }\end{array}$ & MARCKS inhibitor & BIO-11006 & Biomarck & Phase II & [391] & USA \\
\hline $\begin{array}{l}\text { Treat respiratory } \\
\text { illness }\end{array}$ & C5a inhibitor & Ultomiris & Alexion Pharma & Phase III & {$[392,393]$} & Multiple locations \\
\hline $\begin{array}{l}\text { Treat respiratory } \\
\text { illness }\end{array}$ & PD-1 inhibitor & Keytruda & Merck & Phase II & [394] & Spain \\
\hline $\begin{array}{l}\text { Treat respiratory } \\
\text { illness }\end{array}$ & PD-1 inhibitor & Opdivo & $\begin{array}{l}\text { Bristol-Myers } \\
\text { Squibb }\end{array}$ & Phase II & {$[395]$} & France \\
\hline $\begin{array}{l}\text { Treat respiratory } \\
\text { illness }\end{array}$ & $\begin{array}{l}\text { Anti-angiopoietin } 2 \\
\mathrm{mAB}\end{array}$ & LY3127804 & Eli Lilly & Phase II & [396] & USA \\
\hline $\begin{array}{l}\text { Treat respiratory } \\
\text { illness }\end{array}$ & Prostacyclin analog & Ventavis & $\begin{array}{l}\text { Hamad Medical } \\
\text { Corp }\end{array}$ & Phase II & [397] & Qatar \\
\hline $\begin{array}{l}\text { Treat pneumonia / } \\
\text { sepsis }\end{array}$ & PD-1 inhibitor & AiRuika & $\begin{array}{l}\text { Southeast } \\
\text { University }\end{array}$ & Phase II & [398] & China \\
\hline $\begin{array}{l}\text { Prevention, } \\
\text { immunostimulant }\end{array}$ & TLR agonist & PUL-042 & Pulmotect & Phase II & [399] & USA \\
\hline $\begin{array}{l}\text { Anti-viral growth, } \\
\text { imunotherapy }\end{array}$ & NK cell therapy & CYNK-001 & Celularity & Phase I/II & {$[400]$} & USA \\
\hline
\end{tabular}


Table 7

(Continued)

\begin{tabular}{|c|c|c|c|c|c|c|}
\hline Treatment goal & Target & Drug & Company & Stage & Reference & Location \\
\hline Treat organ failure & Sulfated oxysterol & DUR-928 & Durect & Phase II & [401] & USA \\
\hline Reduce organ failure & AT1R inhibitor & Losartan & $\begin{array}{l}\text { University of } \\
\text { Minnesota }\end{array}$ & Phase I & [402] & USA \\
\hline Reduce organ failure & AT1R inhibitor & Diovan & $\begin{array}{l}\text { Radboud } \\
\text { University }\end{array}$ & Phase IV & [403] & Netherlands \\
\hline Reduce organ failure & SGLT2 inhibitor & Farxiga & Astrazeneca & Phase III & [404] & USA \\
\hline $\begin{array}{l}\text { Anti-viral growth, } \\
\text { anti-inflammatory }\end{array}$ & $\begin{array}{l}\text { SK2 selective } \\
\text { inhibitor }\end{array}$ & Yeliva & RedHill & $\begin{array}{l}\text { Phase IIa (IND } \\
\text { Approval) }\end{array}$ & [405] & Israel \\
\hline $\begin{array}{l}\text { Anti-viral growth, } \\
\text { anti-inflammatory }\end{array}$ & $\begin{array}{l}\text { Recombinant human } \\
\text { ACE2 }\end{array}$ & APN01 & $\begin{array}{l}\text { APEIRON } \\
\text { Biologics }\end{array}$ & Phase II & [406] & $\begin{array}{l}\text { Germany, } \\
\text { Austria, } \\
\text { Denmark }\end{array}$ \\
\hline $\begin{array}{l}\text { Anti-viral growth, } \\
\text { anti-inflammatory }\end{array}$ & Calpain inhibitor & BLD-2660 & Blade therapeutics & Phase II & [407] & USA \\
\hline $\begin{array}{l}\text { Anti-viral growth, } \\
\text { anti-inflammatory }\end{array}$ & DHODH Inhibitor & IMU-83 & Immunic & Phase II/III & [408] & $\begin{array}{l}\text { Bulgaria, } \\
\text { Germany }\end{array}$ \\
\hline $\begin{array}{l}\text { Anti-viral growth, } \\
\text { anti-inflammatory }\end{array}$ & DHODH Inhibitor & РTC299 & PTC Therapeutics & Phase II/III & [409] & USA, Spain \\
\hline $\begin{array}{l}\text { Anti-viral growth, } \\
\text { anti-inflammatory }\end{array}$ & $\begin{array}{l}\text { Tyrosine kinase } \\
\text { inhibitor }\end{array}$ & Gleevec & Novartis & Phase III & {$[410]$} & USA \\
\hline $\begin{array}{l}\text { Anti-viral growth, } \\
\text { anti-inflammatory }\end{array}$ & $\begin{array}{l}\text { Antibodies from } \\
\text { recovered patients }\end{array}$ & $\begin{array}{l}\text { Covalescent } \\
\text { plasma therapy }\end{array}$ & Multiple locations & $\begin{array}{l}\text { Phase III/ } \\
\text { Emergency } \\
\text { use }\end{array}$ & [411-422] & $\begin{array}{l}\text { Multiple } \\
\text { locations }\end{array}$ \\
\hline $\begin{array}{l}\text { Anti-viral growth in } \\
\text { cancer patients }\end{array}$ & SUMO inhibitor & TAK-981 & $\begin{array}{l}\text { Takeda } \\
\text { Pharmaceuticals }\end{array}$ & Phase I & [423] & USA, Canada \\
\hline $\begin{array}{l}\text { Neuroprotective, treat } \\
\text { symptoms }\end{array}$ & $\begin{array}{l}\text { Endogenous small } \\
\text { molecule }\end{array}$ & NA-831 & NeuroActiva & Phase II/III & {$[424]$} & USA \\
\hline Treat symptoms & Anti-CTGF mAb & Pamrevlumab & FibroGen & Phase II & {$[425]$} & USA \\
\hline
\end{tabular}

\section{Janus kinase JAK 1 and JAK 2 Inhibitor}

Baricitinib is an inhibitor of both AP 2 associated Protein Kinase 1 and Cyclin G associated Kinase, helps to prevent endocytosis that is needed for viral assembly. Contraindications for its use are increased creatine kinase levels, an absolute neutrophil count less than $1 \times 10^{9}$ cells/L or an absolute lymphocyte count less than $0.5 \times 10^{9}$ cells/L. ${ }^{9}$ Other considerations are the potential reactivation of tuberculosis, hepatitis B, varicella-zoster, herpes simplex and Epstein Barr virus [154].

\section{Nanomedicine for COVID-19 infection management}

It has been suggested that SARS-CoV-2 protein may act like a nanoparticle due to its spherical shape and small size $(2-600 \mathrm{~nm})$ and can be taken up by the host cell using a similar mechanism of endocytosis. If a drug could inhibit the endocytosis of SARS-CoV-2 [155], it would open a door for future research as drugs that can act like nano-medications [156] or active therapeutic agents that can be formulated as carriers for delivering, at the target site, using an effective bio-compatible smart drug nano-carrier [157]. Nanomedicine-based therapy would also minimize the use of excessive COVID-19 drug dosages, which had exhibited adverse effects [155].

Experts have suggested exploring nanomedicine options to manage COVID-19 as a potential therapy using recommended therapeutic agents (synthetic drug, gene, CRISPR-Cas9/gRNA, etc.,) and immuno-acceptable biomaterials to eradicate SARS-COV-2 virus. The tunable drug payload and controlled drug release make nanomedicine suitable for COVID-19 management in a personalized manner [158-162].

\section{Air filtering for COVID-19}

The CDC states that COVID-19 is spread predominantly by large respiratory droplets in the proximity of infected individuals ( $\sim 6$ feet). Studies have shown this accounts for $>70 \%$ spread of the virus. Proper research about the transmission pathways is vital to stop the spread of COVID-19 [163]. A study conducted in an indoor setting has shown that air filtration with particle control technology leads to the reduction of fine $(<2.5$ microns $)$ and ultrafine particles $(<0.25$ 
micron) by $95 \%$. This study has demonstrated the effective elimination of a bacterial bioaerosol surrogate [163].

Proper air filtration methods are necessary to limit the spread of COIVD-19. Particle control technology has more significant advantages over high-efficiency particulate air (HEPA) and other means of filtration of the air, as it relies on local electrostatic field manipulation [164].

\section{Treatment for COVID-19 and Alzheimer's disease and dementia}

Clinical trials targeting viral pathogenesis are still ongoing, therefore a definitive treatment guideline for COVID-19 is not yet finalized. Concerning morbidity and mortality, the disease has highly impacted the elderly population. Those suffering from mental ailments, especially those in nursing homes, will require a different approach when treating COVID-19. Patients are usually on a cocktail of antipsychotics, antidepressants, and disease-modifying antirheumatic drugs. In patients with Alzheimer's disease (AD), delirium resulting from hypoxia will change management since there is a danger of serious adverse reactions with drug interactions [165]. AD treatment such as donepezil, galantamine, rivastigmine (cholinesterase inhibitors, ChEIs), and memantine, are known for their pharmacokinetic properties in the liver's enzymes cytochrome CYP450 (CYP2C8, CYP2D6, CYP3A4). Cholinesterase inhibitors, antidepressants, and antipsychotics have different levels of interaction with both inducers and/or inhibitors of the enzyme. When adding treatment for COVID19, the provider must be aware of the risks involved. Antirheumatic chloroquine, and hydroxychloroquine, inhibit CYP2D6, thus requiring adjusting galantamine and donepezil [165]. Although Azithromycin has the least interaction with CYP450, it shares the same cardiac adverse events as the ChEIs, heart-block, bradycardia, QT interval prolongation as well as Torsades de Pointes. Other drugs like lopinavir and ritonavir inhibit CYP3A4 and induce CYP1A2, a combination has the potential of potentiating systemic effects of galantamine and donepezil, adding a ChEI can lead to bradycardia, a less than desirable outcome in patients with a compromised cardiovascular system [165]. Ritonavir requires special consideration since it interacts with several liver enzymes thus requiring a more meticulous adjustment of medications being used in conjunction. On the other hand, memantine is a preferred drug of choice because of its lower risk of interaction with liver enzymes $[165,166]$. Selective serotonin inhibitors like paroxetine and fluoxetine are both inhibitors of CYP2D6 which can both affect glucose levels when used with disease-modifying antirheumatic drugs chloroquine and hydroxychloroquine. Drugs that can safely be used alongside AD treatment include tocilizumab, ribavirin, favipiravir, especially when treating COVID-19 patients in nursing homes $[165,166]$.

\section{MENTAL HEALTH CONSIDERATIONS}

Isolation, working from home, physical distancing, job loss, and critical illness from the virus, could induce long-term psychological effects in many individuals. Social isolation has been linked to a heightened risk of suicide attempts and suicide [167], and several studies address the connection between job loss and a heightened risk of depression [168, 169], anxiety [169], and increased substance abuse [170, 171].

\section{COVID-19 and mental disorders}

Long-term isolation and loneliness could act as a trigger for mental disorders such as depression and anxiety in some individuals. A study of 3,005 older adults documented that social disconnection and perceived isolation increased the likelihood of depression and anxiety [172]. A separate study of young adults similarly suggested that social isolation can promote feelings of loneliness and a higher probability of experiencing depression [173].

Viral outbreaks specifically appear to contribute to mental health risks, both directly and indirectly. A study in Canada after the severe acute respiratory syndrome (SARS) outbreak revealed that $28.9 \%$ of people exhibited symptoms of post-traumatic stress disorder (PTSD) after being quarantined, and 31.2\% exhibiting symptoms of depression [174]. Another study on the aftermath of SARS showed a positive correlation between length of quarantine and symptoms of PTSD, with increased PTSD symptomology in $14.6 \%$ of the participants. The psychological symptoms were higher among health-care workers relative to others [175]. After the 2013-2016 Ebola epidemic in Africa, several symptoms of mental distress were reported in Ebola survivors, with symptoms of depression being the most prevalent 
at $15-17 \%$. A greater sense of stigmatization and feelings of loneliness were also observed among survivors [176, 177].

Time spent in the ICU is often a traumatic experience, and as such, can more directly increase mental health risks in critically ill COVID-19 patients. A new study on survivors of critical illness admitted to the ICU $(n=116)$ showed that $41.4 \%$ of patients reported at least one long term mental health consequence within six months of discharge. Out of the patients experiencing mental health consequences, anxiety, and depression (or the combination of the two) were the most prominent consequences, at $28.4 \%$ and $20.7 \%$ of patients, respectively. Moreover, the subset of 41 patients receiving mechanical ventilation in this study yielded 22 with long term consequences, a further increase to nearly $54 \%$ [178]. In a separate study that included ICU patients receiving mechanical ventilation, signs of anxiety and depression were evident at three months follow up in $\sim 30 \%$ and $\sim 21 \%$, respectfully, as well as PTSD in $29.9-34.3 \%$ of patients [179]. The scale of negative psychological aftermath due to COVID-19 has yet to be determined; however, some ramifications will undoubtedly arise. A small surveillance study in the UK $(\mathrm{N}=125)$ found that $31 \%$ of the patients displayed an altered mental status in the form of a psychiatric disorder, including newonset psychosis $(43 \%)$, neurocognitive (dementia like) disorder (26\%), and an affective disorder (17\%) [89].

Health care workers on the front-line with COVID19 patients will experience an especially large strain, both mentally and physically. Especially in high-risk areas [180], health care workers have displayed symptoms of anxiety [180-182], depression, insomnia [180, 182], and distress from their work environment [181]. Surveillance of psychological conditions following this epidemic as well as measures to minimize psychological distress in the general public may represent an implementable preventive measure now. Especially as experienced by healthcare and other front-line workers.

\section{COVID-19 and domestic abuse}

As isolation and lockdown all over the world has been put in place, a perfect storm of stressors for the individual could be a trigger for domestic abuse and violence-related behavior in the home. It could be harder for the abusee to get away from the situation. An increase in domestic violence have currently been reported on a large scale all over the globe as people are spending more time at home. In Australia, a $75 \%$ increase in online searches for domestic abuse has been reported. In Wuhan, China, domestic abuse raised a threefold in February. France and the US reported an increase of between $21 \%$ to $36 \%$ in domestic violence complaints during the ongoing pandemic [172]. In the UK, calls, emails, and website visits for a domestic violence charity, increased $97 \%$, $185 \%$, and $581 \%$, respectively [183]. In the Czech Republic, there were a doubling of calls to a hot line for abuse victims during the lockdown. In poorer countries like Ukraine and Armenia, a decrease of calls was seen during the lockdown, which could be linked to a lack of internet connection, proximity to the abusive partner, and the referral of the abusees by others, like pharmacy employees or friends [184].

Preventative measures must be put into place, so that people in abusive households have a way to get the help they need. Several countries, including Norway, Germany, France, Italy, and Spain have created a codeword, "MASK 19", which can be uttered in supermarkets and pharmacies as a signal for help from domestic violence [183]. Another preventative measure could be financial support for people without a job to get out of abusive households, as many people have lost their jobs due to the pandemic and will not be able to support themselves.

\section{COVID-19 and Alzheimer's disease and dementia.}

Age and comorbid medical conditions have consistently been the most impactful factors correlated with poor prognosis, hospitalization, and mortality in COVID-19 patients. Alzheimer's disease-related dementias (ADRD) elderly patients are an especially vulnerable group because they often have underlying comorbidities [190, 195, 196]. APOE $\varepsilon 4$, one of the genotypes that puts people at a greater risk of developing ADRD, could possibly be linked to the severity of COVID-19 [185]. Yet more research is called for before concluding that $A P O E \varepsilon 4$ genotype influences disease severity in COVID-19.

Individuals with dementia are also more likely to suffer from strokes, atherosclerosis, diabetes, insomnia, incontinence, fractures, and pneumonia, compared to individuals of the same age without dementia [197], which again puts them at a higher risk for developing severe COVID-19 symptoms if they contract the virus, thus leading to a higher mortality rate in this patient population [190]. 
People suffering from ADRD are in a specifically vulnerable positions because they heavily rely on others, like family or professional caregivers, for their activities of daily living. This COVID-19 pandemic further exacerbates their vulnerability, due to the synergism of both morbidity and mortality from COVID-19, ADRD and the effects of the pandemic on the social support networks and the healthcare system on which they depend [190]. ADRD was found to be the most common pre-existing condition among patient deaths related to COVID-19 that amounted to $49.5 \%$ of all deaths of care home residents in England and Wales, between March 2 and June 12, 2020 [186]. $40 \%$ (about 40,000 individuals) of deaths related to COVID-19 in the US have happened in long-term care facilities or nursing homes. It is estimated that more than 2.4 million patients live in long-term care facilities nationwide; this represents a mortality rate of about 450 individuals per day since March to June 2020 [187, 188]. The National Center for Health Statistics (2016) estimates that the percentage of residents of residential care communities with ADRD represents $41.9 \%$ and the percentage of nursing home residents with ADRD is 47.8\% [189].

The COVID-19 pandemic is affecting services and daily life such as: meals on wheels, either disrupting or delaying it due to increased demand or closure; unavailability of family or professional caregivers due to increased demand, illness, or required isolation; personal savings and investments, with a heightened risk of financial adversity due to the economic slowdowns of the pandemic; increased exposure and risk of infection due to reliance on public transportation services; increased mental health afflictions due to increased isolation and disruption of group activities; the inability to use the technology to stay socially connected and access services, including health care, due to cognitive impairment [190].

Professionals have encountered difficulties while working with adult institutionalized patients, such as isolation induced by the limitation of visits, the shortage of protective equipment and tests, the sustainability of overworking professionals, family members' concern for the residents, complex household situations, and difficulties in accessing care. These challenges are consistent with the social effects of the pandemic. However, the main challenges of individuals with cognitive impairment seems to be understanding and remembering the recommended measures needed to avoid spread and contraction of the virus [190, 194, 198].
Compliance of recommendations from public health authorities to reduce the transmission and spread of COVID-19 may be unachievable for different reasons in ADRD patients. For example, in milder dementias, it might be hard to follow due to apathy or depression. In more severe dementia, the severity of their short-term memory loss and overall cognitive impairment means they are unable to understand, comprehend, or remember most of these recommendations [190].

\section{NUTRITIONAL CONSIDERATIONS}

Nutrition intervention, medical nutrition therapy, close management, and timely follow up are all part of the overall approach to treating COVID-19 individuals from the ICU setting to the medicine ward. Appropriate nutritional therapies, especially in the frail elderly, immunocompromised, and individuals with co-morbidities who may already be malnourished or at risk of malnutrition, can ensure survival as well as better and shorter recovery from this illness [191].

Crucial steps for nutrition intervention and management of COVID-19 patients:

1. Screen and check for malnutrition. Patients at risk for worse outcomes and higher mortality following infection with SARS-Cov-2, namely older adults and individuals with co-morbidities must be screened and checked for malnutrition [191]. A registered dietitian (RD) is a trained professional who can identify these individuals by using one of several validated malnutrition screening tools, i.e., Malnutrition Screening Tool or NRS-2002 (Nutrition Risk Screening) [191]. The RD will also perform a nutrition focused physical exam, observing for physical signs of malnutrition, i.e., orbital/temporal wasting, clavicle protrusions, etc. If a patient is determined to be at risk or malnourished, a global nutrition assessment should be conducted to begin interventions on improving patients' nutritional status.

2. In the ICU, vented individuals will need to be provided nutrition support via a nasogastric tube or post-pyloric tube (for prone positioning) [123] within 24-36 hours of admission or 12 hours of intubation. Trophic feeding at $10-20 \mathrm{ml} /$ hour with an isotonic formula, i.e., $1-1.5 \mathrm{kcal} / \mathrm{ml}$ with a high nitrogen con- 


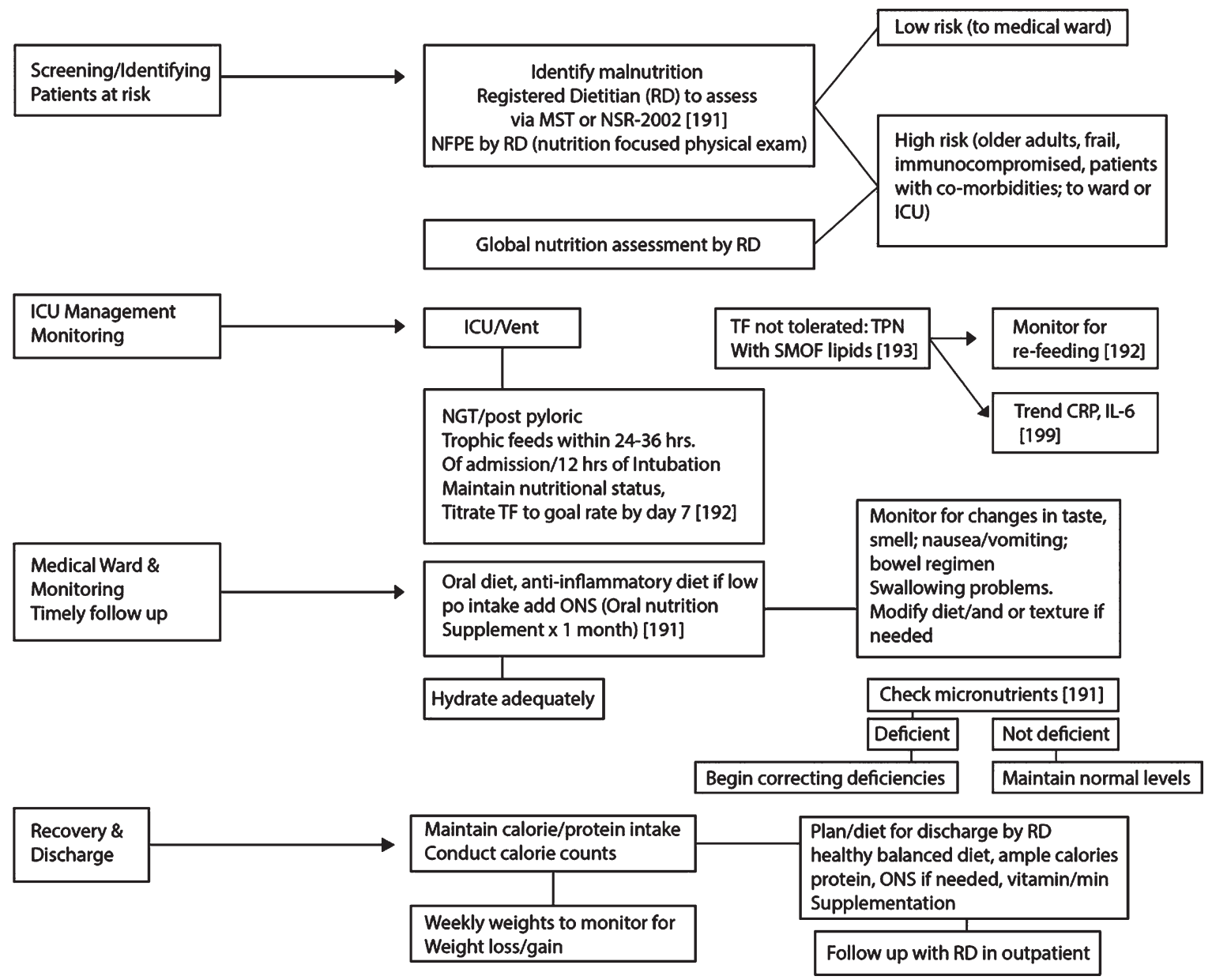

Fig. 6. Nutritional Algorithm.

tent is suggested. During the first week, caloric goals are $15-20 \mathrm{kcal} / \mathrm{kg}$ of actual weight, which is about $70-80 \%$ of estimated needs. The end of the first week should achieve this goal. By week two, caloric goals are $25-35 \mathrm{kcal} / \mathrm{kg}$ or $(\mathrm{REE} \times 1.2-2.0)$ and by day $15,32 \mathrm{kcal} / \mathrm{kg}$ or $(\mathrm{REE} \times 1.4)$. Protein goals are aimed at $1.2-2.0 \mathrm{gm} / \mathrm{kg}$ of actual weight (day 0-4) and 1.5-2.0 gm/kg (>day 4) [192]. For obese patients, the goal is $2.0-2.5 \mathrm{gm} / \mathrm{kg}$ of ideal weight. If enteral nutrition is not tolerated, parenteral nutrition is an option. Use Soy/MCT/Olive oil/Fish oil lipids and at least $0.7 \mathrm{gm} \mathrm{fat} / \mathrm{kg}$ to achieve anti-inflammatory benefits [192, 193]. Refer to Fig. 6 for an overview of the nutritional considerations.

3. Post ICU/medicine ward. Energy needs are 25$35 \mathrm{kcal} / \mathrm{kg}$ with protein goals of $1.5-2.0 \mathrm{gm} / \mathrm{kg}$; obese individuals $2.0-2.5 \mathrm{gm} / \mathrm{kg}$ of ideal weight [199]. The oral diet should not be therapeutically restrictive as many patients post ICU do not eat well. Texture modification should be considered for the elderly with chewing/swallowing difficulty. Monitoring patients for loss of taste/smell, nausea, vomiting, diarrhea, and constipation is necessary as all can affect oral intake. Nursing staff to assist with feeding if the patient cannot feed him/herself. Patients should walk within their rooms or the ward if possible, to help improve gut motility. An anti-inflammatory diet would be beneficial with ample servings of fatty fish, fruits, vegetables, beans/legumes, olive oil, nuts, and seeds. Patients that cannot meet caloric/protein goals should be provided with an oral nutrition supplement with 400 calories $/ 30 \mathrm{gm}$ protein 
daily for at least one month for repletion [191]. A calorie count can be initiated to verify actual oral intake, and if poor intake is seen, enteral nutrition can be an option to meet caloric/protein goals temporarily. Adequate hydration should be emphasized [191].

4. Check micronutrients and correct deficiencies, if any. In general, low levels of intakes of micronutrients such as vitamin A, E, B6 and $\mathrm{B} 12$, zinc, and selenium have been associated with adverse clinical outcomes during viral infections [191]. Some reports show that vitamin C has antimicrobial effects and vitamin D deficiency has been related to several viral diseases. At this time, there are insufficient data for additional supplementation over the standard unless vitamin/mineral deficiency is seen [191, 192].

5. Monitoring COVID-19 patients. Monitor for signs of re-feeding syndrome frequently and replete magnesium, potassium, and phosphorus if depleted in both ICU and post ICU patients if inadequate energy/calorie intake has been suspected for $>7$ days. Slow feeding rate with enteral nutrition or parenteral nutrition $(25 \%$ of caloric goals) is necessary [192]. Monitor CRP and IL-6 levels for trends to assess the degree of catabolism and inflammation [199].

6. Weekly/bi-weekly standing scale weights by nursing staff are crucial to monitor for weight loss/gain and to intervene quickly before further weight fluctuation is seen.

The road to recovery for COVID-19 individuals starts with adequate nutrition and correcting their preexisting malnutrition by providing timely and proper nutrition support, enterally, parenterally, or orally. A solid plan before discharging home by the RD will need to be reviewed with the patient and family, discussing the importance of eating balanced meals with adequate calories, protein, anti-inflammatory foods, and use of oral nutrition supplement and any vitamin and mineral supplementation. Sensitivity to the socioeconomic status of the individual is necessary to identify food insecurity if any. Providing resources to the individual for meal delivery services or obtaining food is beneficial as many may face difficulty purchasing and preparing nutritious meals after the hospital stay. Post-discharge referrals to an outpatient RD should be considered for individuals recovering from COVID-19 who may benefit from ongoing nutritional follow up.

\section{REHABILITATION CONSIDERATIONS}

Post-COVID-19 patients present some unique challenges to the rehabilitation team, in addition to multiple typical sequelae of prolonged bedrest and mechanical ventilation.

Patients who have experienced prolonged bedrest and mechanical ventilation typically suffer from profound muscle weakness, balance deficits, orthostatic hypotension, respiratory muscle weakness which mimics a restrictive lung disease, deep vein thrombosis, decubitus ulcer, joint contractures, postintubation dysphagia, etc.

In addition to the above, patients with COVID-19 in the acute rehabilitation setting have been noted to suffer from a high incidence of dysautonomia (resulting in both orthostatic hypotension and/or severe hypertension and tachycardia), severely reduced activity tolerance, acute stress reaction related to ICU care and intubation (which can be triggered by exertion dyspnea), and diffuse cognitive impairment [200].

Rehabilitation efforts require a multi-disciplinary approach including physical therapy, occupational therapy, speech and language pathologists, and respiratory technicians. Initial efforts are focused on the respiratory muscle strengthening and secretion/dysphagia management [201]. The circulatory system is addressed initially with postural challenges and tends to respond well to this over time. Mobility and functional restoration are achieved with low intensity muscle and aerobic strengthening regimens, which can be intensified over the course of recovery [201].

\section{GENERAL COMPLICATIONS}

The most common short-term complications of SARS-CoV-2 infection observed in severe cases included ARDS (100.0\% of patients with available ABG data) and respiratory failure (83.3\%) [109]. As seen in a study of 21 hospitalized patients in Wuhan, with laboratory-confirmed moderate to severe COVID-19. Less common complications among the severe cases in that study, included secondary infections $(27.3 \%)$, acute cardiac injury $(9.1 \%)$ and hypoxic encephalopathy $(18.2 \%)$, acute kidney injury $(18.2 \%)$, shock $(9.1 \%)$ acute liver injury (9.1\%) [109], and rhabdomyolysis [202]. Other complications reported: 
- Acute hemorrhagic necrotizing encephalopathy is a rare viral complication that has been associated with intracranial cytokine storms, that results in a breakdown of the bloodbrain-barrier, without direct viral invasion or demyelination. It presents itself as a hypo attenuated CT image and a T2 FLAIR hyperintense signal, with internal hemorrhage on MRI located most commonly in the thalamus. It can also affect the basal ganglia, brainstem, cerebral white matter, and cerebellum [203]. This case was reported in a female airline worker in her fifties, positive for COVID-19.

- Fulminant myocarditis (FM), is a rare syndrome that presents with cardiac inflammation and displays a mortality rate of approximately $40-70 \%$. FM can be categorized by lymphocytic, eosinophilic, giant cell myocarditis, and sarcoid heart disease. The lymphocytic can be further divided into an infective and noninfective origin. Viral etiology is suspected in most of cases, and biopsy studies of patients with acute myocarditis in Europe indicate that viral etiology ranges between $37.8 \%$ and $77.4 \%$ Death was associated with cardiac damage induced by a cytokine storm, which is the pathophysiological mechanism in FM. FM was reported in an observational study in 120 patients from Wuhan with severe COVID-19, who exhibited increased IL-6 levels and needed ICU admission. Positive COVID-19 patients with increased Troponin I and new-onset arrhythmias should be followed up closely to prevent cardiac complications [204].

- Spontaneous pneumomediastinum is a selflimiting condition that can cause severe circulatory and respiratory disease. This case was reported in a 38-year-old man from Wuhan, China, who presented exertional angina, palpitations, and respiratory wheezing. On day 11, the patient's chest CT was positive for multiple, bilateral ground-glass opacities with consolidation, and interlobular septal thickening was also observed. Spontaneous pneumomediastinum and subcutaneous emphysema are additionally seen. The exact mechanism of the development of the pneumomediastinum is still unknown [205].

- Acute renal failure, a retrospective laboratory analysis of kidney function, focused on eGFR in 85 COVID-19 patients, showed 23 patients
(27.06\%) exhibiting acute renal failure, more frequently in elderly patients $(65.22 \%$ versus $24.19 \%, p<0.001$ ), or in patients with comorbidities such as hypertension or coronary heart disease $(69.57 \%$ versus $11.29 \%, p<0.001)$. The damage caused by SARS-CoV-2 infection is due to the induction of severe acute tubular necrosis and lymphocyte infiltration. In their study, Bo Diao and colleagues confirmed the direct infectivity of kidney tubules by SARS-CoV-2, by the viral N-antigens immunohistochemistry. The study also revealed a strong presence of $\mathrm{CD}^{+} 8^{+}$macrophages in the tubule interstitium as well as deposition of the complement membrane attack complex (MAC, C5b-9) on tubules, which could cause damage [206].

Studies have shown that COVID-19 re-infection is not viable for 7 to 10 days after resolution of the first occurrence. A study in Rhesus monkeys presented no viral replication in all primary tissue samples 5 days after re-infection. This could indicate that a SARS-CoV-2 primary infection could be protective to subsequent occurrences [207].

Cell-mediated and humoral immunity are involved in SARS-CoV-2 infection. To this date, protection from re-infection and a definitive viral clearance timeline has not been clearly established. Based on a study of 11 COVID-19 laboratory-confirmed patients who had experienced two separate symptomatic occurrences, it was hypothesized that either a viral re-infection had occurred or that viral reactivation from tissue reservoirs had taken place [208]. In the case of healthy healthcare workers in settings of prolonged exposure to the virus, mild symptoms can point to a re-infection. In comparison, a re-activation in vulnerable individuals at low risk of infection could have resulted from less than ideal SARS-CoV-2 treatment and management leading to viral replication [208]. Positive RT-PCR test results from recovered patients might have been confounded by cross contamination from another coronavirus, whereas false positive results could have been due to inappropriate sample collection [207].

The long-term complications among COVID-19 survivors are not yet available [48]. The mortality rates for cases globally are $0.1 \%$ in patients' $\leq 9$ years old to $15 \%$ in patients $\geq 80$ years old, emphasizing the impact of age on this disease [48, 209]. 


\section{DISCUSSION AND CONCLUSION}

The vast majority of COVID-19 patients undergo different manifestations of disease without a particular order. They may present with ARDS, cytokine storm, acute hypercoagulable state, and autonomic dysfunction, presentations, which must be managed by an array of multidisciplinary teams, including but not limited to infectious disease, pulmonologists, cardiologists, neurologists, nephrologists, gastroenterologists, pain management specialists, psychiatrists, ophthalmologists, internists/hospitalists, orthopedic teams, laboratory medicine and immunologists, as well as dieticians, nursing staff, rehabilitation specialists, and clinical trial teams. Communication, coordination, and collaboration in a timely fashion (C3T), both within care units and when consulting with more experienced teams externally, can enhance patient care and allow prompt treatment evaluation. In lieu of effective treatments, preventive measures, such as physical distancing, facemasks, personal hygiene including thorough washing of hands, and community testing, are highly recommended. Since SARS-CoV-2 may have a substantially higher infectious rate than previously believed both in young children and adults, effective and population-inclusive testing protocols should be put into place to quantify its spread in the population. Otherwise it is difficult to assess actual hospitalization rates without proper data, which is critical, as it will determine how quickly we can return to normality.

COVID-19 has had an enormous impact on physical and mental health, as self-isolation has not only contributed to economic hardship but has possibly also led to higher morbidity and mortality rates from anxiety, suicide, depression, PTSD, and other mental illnesses. The fear of becoming infected may itself discourage those with underlying comorbidities requiring medical attention, such as cardiovascular events, to seek medical care thus potentially aggravating their condition and risking death. Fluid management has shown to have a significant positive impact in the recovery of COVID-19 patients. Conservative fluid management in patients who are prone to pulmonary edema, as well as high risk of developing cardiomyopathy that exacerbates fluid overload, is highly recommended. It must also be noted that pulmonary management in those patients becomes a challenge and extremely difficult to maintain. Dialysis in COVID-19 patients should also be considered as a therapeutic tool for patients with kidney disease and end-stage renal failure since cytokine-filtering dialysis filters are already available.

The authors suggest that if the patients have loss of consciousness or an altered mental status, then 1) acute hypercapnia, 2) sepsis, and/or 3) seizures, should be suspected and ruled out. Electroencephalography could serve as a tool in assessing the brain function in COVID-19 patients.

It is also important to monitor CRP and IL-6 trends for cytokine storm, and close monitoring of infection symptoms to prevent potential sepsis and subsequent organ failure. Acute hypercoagulable state should be predicted and managed to avoid coagulopathy, stroke, thrombotic events, including limb thrombosis, which could cause compartment syndrome, as well as pulmonary/organ embolism.

Proper pain management in COVID-19 patients is essential. Providers need to be aware of when, and how much pain medications/sedatives to give the patient, since this could have an impact on their physiology and cognition which could potentially mask or cause metabolic encephalopathy.

A comprehensive approach to the management of COVID-19 individuals should involve various treatment modalities. These include a dietary assessment and treatment to effectively reduce complications and improve both short and long-term prognosis essential to ensuring survival, especially in the recovery phase. Furthermore, in order to prevent edema, refeeding syndromes, or micronutrient deficiencies, long-term stay patients in the ICU settings should be routinely monitored for nutritional deficiencies. A review of the literature indicates that common causes of death include cardiovascular, pulmonary, and other organ complications. Therefore, in those COVID19 patients with underlying comorbidities (e.g., cardiovascular, pulmonary, ADRD, and immunocompromised), it becomes imperative to identify the patients with a high mortality risk. This should be done in order to focus on precise protective measures and management strategies to mitigate negative outcomes in those at risk or those already infected with the virus.

There is an urgent need for a comprehensive, national, and unified guideline for prevention, diagnosis, and treatment of COVID-19. The absence of such guidelines in the US and globally, in addition to the miscommunication and sub-optimal management of the disease by the WHO have created chaos and confusion among healthcare professionals, poli- 
cymakers, healthcare institutions, caregivers, and the general public. Unfortunately, the lack of attention to the science and science-based policy in a timely fashion has contributed to the spread of conspiracy theories, misinformation, and mismanagement of the pandemic in the US and abroad.

These have impacted, and will continue to impact, the duration, management, and science-based policy of the pandemic prevention and control. In light of conflicting and inconclusive data and the fact that the main mechanism of viral entry is by the ACE2 receptor, not only do we believe that HCQ may not be effective in the prevention and or treatment of COVID-19, but it could also cause side effects. Hence, we recommend facemasks, physical distancing, and personal hygiene as preventative measures, and other promising medications/therapeutic approaches listed in this paper be used for therapy.

Patients with ADRD need special attention and care during a pandemic to be able to follow the healthcare authorities' recommendations (i.e., CDC, national/local healthcare authorities). Due to poor cognition which result in the incapability of effective physical distancing, hand washing, and correct use of facemasks, they are at a higher risk of contracting COVID-19. Such individuals may not remember their medication, timing of their treatments and follow ups, or understand what is happening around them and to them, which could worsen their overall medical and mental conditions. Therefore, there is a great need for vaccine-based therapy. There is evidence showing the seasonal flu-vaccine and the Pneumococcal vaccine decreases the risk of acquiring AD. More studies are required to establish such connections between COVID-19 and AD as well as other neurodegenerative disorders [210].

Young children, showing little or no symptoms themselves if infected, might also need extra attention and follow up in regards to hand washing and physical distancing to prevent further spread of the virus.

Plenty of resources have been allocated for containment measures, specifically in the development of vaccines. Yet the possibility of not having a functional vaccine, or having one in a timely manner, must also be considered [211]. Therefore, we not only have to pursue vaccine therapy as mentioned in the paper, but also refine our therapeutic protocols including immunotherapy. Such therapies can include the administration of protective antibodies or serum from recovered patients, as well as modulation of beneficial immune responses and blunting of detrimental immune activity via immunosuppression. In light of newly discovered viral mutations, discussed in this paper, we recommend enhancing B-cell and T-cell response in order to protect and prevent COVID-19 patients from potential re-infection. Novel diagnostics and therapeutics like nanomedicine, discussed in this paper, should be considered for future drugs and diagnostics development of this complex disease.

We believe patients would have a better outcome from well-coordinated, interdisciplinary, specialized and synchronized teams. Communication, coordination, and collaboration in a timely fashion (C3T), could impact the outcome of care for these patients. Through this systematic approach, their underlying conditions could be controlled, disease-related symptoms could be predicted, prevented, detected and treated on time, and before serious complications arise. The authors would like to emphasize the importance of global preparedness for the pandemic of mental health as the result of the COVID-19 so as to respond accordingly and effectively. These include science-based policies, public education on the state of science, and funding scientific research. The use of contactless mass-screening devices, such as thermal imaging cameras, are recommended in indoor public venues. There is a greater need to understand the importance of the transmission pathway of COVID19 to inform the general population better and make practical guidelines to manage better and limit the spread.

In this paper we have reviewed 28 vaccine clinical trials, several emerging diagnostic tools and therapeutics for COVID-19, which paints a promising picture for the management of this novel disease.

\section{ACKNOWLEDGMENTS}

The authors are acknowledging the graphic design and illustration work done by Mr. Shawn, Ms. Brianna, Shanna McDonald, Dr. Joe Bolanos, and Dr. Babak Kateb. We want to thank Dr. Waguih W. IsHak, Dr. Robert Imani and Dr. Manoucher Rashti for their indirect contributions to this paper. We would like to acknowledge the Brain Mapping Foundation (BMF) and the Society for Brain Mapping and Therapeutics (SBMT) for their operational and financial support. We thank Ms. Maleheh Samsami Shirzad/Kateb because her successful care of her COVID-19 among 100s of other patients by this team provided a remarkable insight to the disease, which is now being shared by our team. We would like to 
thank the nursing and medical staff at all of the affiliated hospitals in this paper: USC, UCI, UCLA, NYU, Cornell University, Stanford University, Loma Linda University Lafayette Hospital, and specially the staff at the Cedars-Sinai Medical Center. This work is dedicated to our patients, our families, colleagues, and front-line workers to better the understanding of COVID-19.

Authors' disclosures available online (https:// www.j-alz.com/manuscript-disclosures/20-0831r2).

\section{REFERENCES}

[1] World Health Organization, Coronavirus disease (COVID-19) Situation Report - 191, https://www.who. int/docs/default-source/coronaviruse/situation-reports/ 20200729-covid-19-sitrep-191.pdf?sfvrsn=2c327e9e_2, Last Update 29 July 2020, Accessed 29 July 2020.

[2] He F, Deng Y, Li W (2020) Coronavirus disease 2019 (COVID-19): What we know? J Med Virol 92, 719-725.

[3] CDC, Demographic Trends of COVID-19 cases and deaths in the US reported to CDC, https://www.cdc.gov/coviddata-tracker/\#demographics, Last Update 27 June 2020, Accessed 27 June 2020.

[4] CDC, Characteristics of Persons Who Died with COVID19 - United States, February 12-May 18, 2020, Last Update 17 July 2020, Accessed 27 July 2020.

[5] Heald-Sargent T, Muller WJ, Zheng X, Rippe J, Patel AB, Kociolek LK (2020) Age-related differences in nasopharyngeal severe acute respiratory syndrome coronavirus 2 (SARS-CoV-2) levels in patients with mild to moderate coronavirus disease 2019 (COVID-19). JAMA Pediatr, doi: 10.1001/jamapediatrics.2020.3651

[6] Szablewski CM, Chang KT, Brown MM, Chu VT, Yousaf AR, Anyalechi N, Aryee PA, Kirking HL, Lumsden M, Mayweather E, McDaniel CJ, Montierth R, Mohammed A, Schwartz NG, Shah JA, Tate JE, Dirlikov E, Drenzek C, Lanzieri TM, Stewart RJ (2020) SARS-CoV-2 transmission and infection among attendees of an overnight camp - Georgia, June 2020, https:// www.cdc.gov/mmwr/volumes/69/wr/mm6928e1.htm?s_ cid=mm6928e1_w, Last Updated 31 July 2020, Accessed 31 July 2020.

[7] Huang C, Wang Y, Li X, Ren L, Zhao J, Hu Y, Zhang L, Fan G, Xu J, Gu X, Cheng Z, Yu T, Xia J, Wei Y, Wu W, Xie X, Yin W, Li H, Liu M, Xiao Y, Gao H, Guo L, Xie J, Wang G, Jiang R, Gao Z, Jin Q, Wang J, Cao B (2020) Clinical features of patients infected with 2019 novel coronavirus in Wuhan, China. Lancet 395, 497-506.

[8] Rothan HA, Byrareddy SN (2020) The epidemiology and pathogenesis of coronavirus disease (COVID-19) outbreak. J Autoimmun 109, 102433.

[9] Wang W, Tang J, Wei F (2020) Updated understanding of the outbreak of 2019 novel coronavirus (2019-nCoV) in Wuhan, China. J Med Virol 92, 441-447.

[10] Chen H, Guo J, Wang C, Luo F, Yu X, Zhang W, Li J, Zhao D, Xu D, Gong Q, Liao J, Yang H, Hou W, Zhang Y (2020) Clinical characteristics and intrauterine vertical transmission potential of COVID-19 infection in nine pregnant women: A retrospective review of medical records. Lancet 395, 809-815.
[11] Chen Y, Peng H, Wang L, Zhao Y, Zeng L, Gao H, Liu Y (2020) Infants born to mothers with a new coronavirus (COVID-19). Front Pediatr 8, 104.

[12] Lai C-C, Shih T-P, Ko W-C, Tang H-J, Hsueh P-R (2020) Severe acute respiratory syndrome coronavirus 2 (SARSCoV-2) and coronavirus disease-2019 (COVID-19): The epidemic and the challenges. Int J Antimicrob Agents 55, 105924.

[13] Li Q, Guan X, Wu P, Wang X, Zhou L, Tong Y, Ren R, Leung KSM, Lau EHY, Wong JY, Xing X, Xiang N, Wu Y, Li C, Chen Q, Li D, Liu T, Zhao J, Liu M, Tu W, Chen C, Jin L, Yang R, Wang Q, Zhou S, Wang R, Liu H, Luo Y, Liu Y, Shao G, Li H, Tao Z, Yang Y, Deng Z, Liu B, Ma Z, Zhang Y, Shi G, Lam TTY, Wu JT, Gao GF, Cowling BJ, Yang B, Leung GM, Feng Z (2020) Early transmission dynamics in Wuhan, China, of novel coronavirus-infected pneumonia. N Engl J Med 382, 1199-1207.

[14] Chen N, Zhou M, Dong X, Qu J, Gong F, Han Y, Qiu Y, Wang J, Liu Y, Wei Y, Xia Ja, Yu T, Zhang X, Zhang L (2020) Epidemiological and clinical characteristics of 99 cases of 2019 novel coronavirus pneumonia in Wuhan, China: A descriptive study. Lancet 395, 507-513.

[15] Schoeman D, Fielding BC (2019) Coronavirus envelope protein: Current knowledge. Virol J 16, 69.

[16] Wu C, Liu Y, Yang Y, Zhang P, Zhong W, Wang Y, Wang Q, Xu Y, Li M, Li X, Zheng M, Chen L, Li H (2020) Analysis of therapeutic targets for SARS-CoV-2 and discovery of potential drugs by computational methods. Acta Pharm $\operatorname{Sin} B$ 10, 766-788.

[17] Li F (2016) Structure, function, and evolution of coronavirus spike proteins. Annu Rev Virol 3, 237-261.

[18] Zhou P, Yang X-L, Wang X-G, Hu B, Zhang L, Zhang W, Si H-R, Zhu Y, Li B, Huang C-L, Chen H-D, Chen J, Luo Y, Guo H, Jiang R-D, Liu M-Q, Chen Y, Shen X-R, Wang X, Zheng X-S, Zhao K, Chen Q-J, Deng F, Liu L-L, Yan B, Zhan F-X, Wang Y-Y, Xiao G-F, Shi Z-L (2020) A pneumonia outbreak associated with a new coronavirus of probable bat origin. Nature 579, 270-273.

[19] Lu R, Zhao X, Li J, Niu P, Yang B, Wu H, Wang W, Song H, Huang B, Zhu N, Bi Y, Ma X, Zhan F, Wang L, Hu T, Zhou H, Hu Z, Zhou W, Zhao L, Chen J, Meng Y, Wang J, Lin Y, Yuan J, Xie Z, Ma J, Liu WJ, Wang D, Xu W, Holmes EC, Gao GF, Wu G, Chen W, Shi W, Tan W (2020) Genomic characterisation and epidemiology of 2019 novel coronavirus: Implications for virus origins and receptor binding. Lancet 395, 565-574.

[20] Zhao Y, Zhao Z, Wang Y, Zhou Y, Ma Y, Zuo W (2020) Single-cell RNA expression profiling of ACE2, the receptor of SARS-CoV-2. Am J Respir Crit Care Med, doi: 10.1164/rccm.202001-0179LE

[21] Hamming I, Timens W, Bulthuis M, Lely A, Navis G, van Goor H (2004) Tissue distribution of ACE2 protein, the functional receptor for SARS coronavirus. A first step in understanding SARS pathogenesis. J Pathol 203, 631-637.

[22] Xia H, Lazartigues E (2008) Angiotensin-converting enzyme 2 in the brain: Properties and future directions. J Neurochem 107, 1482-1494.

[23] Baig AM, Khaleeq A, Ali U, Syeda H (2020) Evidence of the COVID-19 virus targeting the CNS: Tissue distribution, host-virus interaction, and proposed neurotropic mechanisms. ACS Chem Neurosci 11, 995-998.

[24] Fan C, Li K, Ding Y, Lu WL, Wang J (2020) ACE2 expression in kidney and testis may cause kidney and testis damage after 2019-nCoV infection. medRxiv, doi: $10.1101 / 2020.02 .12 .20022418$ 
[25] Teoh K-T, Siu Y-L, Chan W-L, Schlüter MA, Liu C-J, Peiris JSM, Bruzzone R, Margolis B, Nal B (2010) The SARS coronavirus E protein interacts with PALS1 and alters tight junction formation and epithelial morphogenesis. Mol Biol Cell 21, 3838-3852.

[26] Wu Q, Zhang Y, Lü H, Wang J, He X, Liu Y, Ye C, Lin W, Hu J, Ji J, Xu J, Ye J, Hu Y, Chen W, Li S, Wang J, Wang J, Bi S, Yang H (2003) The E protein is a multifunctional membrane protein of SARS-CoV. Genomics Proteomics Bioinformatics 1, 131-144.

[27] CDC/Alissa Eckert MDH, MAMS, Public Health Image Library ID 23312, CDC, https://phil.cdc. gov/Details.aspx?pid=23312, Posted 2020, Accessed 26 July 2020.

[28] The Editors of Encyclopedia Britannica, Coronavirus, https://www.britannica.com/science/coronavirus-virusgroup, Last Update 20 April 2020, Accessed 26 July 2020

[29] Hoffmann M, Kleine-Weber H, Schroeder S, Krüger N, Herrler T, Erichsen S, Schiergens TS, Herrler G, Wu N-H, Nitsche A, Müller MA, Drosten C, Pöhlmann S (2020) SARS-CoV-2 cell entry depends on ACE2 and TMPRSS2 and is blocked by a clinically proven protease inhibitor. Cell 181, 271-280.e278.

[30] Sigrist CJ, Bridge A, Le Mercier P (2020) A potential role for integrins in host cell entry by SARS-CoV-2. Antiviral Res 177, 104759.

[31] Tang X, Wu C, Li X, Song Y, Yao X, Wu X, Duan Y, Zhang H, Wang Y, Qian Z, Cui J, Lu J (2020) On the origin and continuing evolution of SARS-CoV-2. Natl Sci Rev 7, 1012-1023.

[32] Forster P, Forster L, Renfrew C, Forster M (2020) Phylogenetic network analysis of SARS-CoV-2 genomes. Proc Natl Acad Sci U S A 117, 9241.

[33] Bedford T, Reher R, Handfield J, Hodcroft E, Sibley T, Huddleston J, Lee J, Fay K, Bell S, Megill C, Potter B, Sagulenko P, Callender C, Ilcisin M, Moncla L, Black A, Brito A, Grubaugh N. Nextstrain: Real-time tracking of pathogen evolution, Nextstrain, https://nextstrain.org, Accessed 18 June 2020.

[34] GISAID, Clade and lineage nomenclature aids in genomic epidemiology studies of active hCoV-19 viruses, https://www.gisaid.org/references/statementsclarifications/clade-and-lineage-nomenclature-aids-ingenomic-epidemiology-of-active-hcov-19-viruses/, Last Updated 4 July 2020, Accessed 27 July 2020.

[35] GISAID, Genomic epidemiology of hCoV-19, https://www.gisaid.org/epiflu-applications/next-hcov19-app/, Last Updated 27 July 2020, Accessed 27 July 2020.

[36] CDC, Coronavirus Disease 2019 (COVID-19), https://www.cdc.gov/coronavirus/2019-ncov/symptomstesting/symptoms.html, Last Updated 13 May 2020, Accessed 20 May 2020.

[37] Bouaziz JD, Duong TA, Jachiet M, Velter C, Lestang P, Cassius C, Arsouze A, Domergue Than Trong E, Bagot M, Begon E, Sulimovic L, Rybojad M (2020) Vascular skin symptoms in COVID-19: A French observational study. $J$ Eur Acad Dermatol, doi: 10.1111/jdv.16544

[38] Magro C, Mulvey JJ, Berlin D, Nuovo G, Salvatore S, Harp J, Baxter-Stoltzfus A, Laurence J (2020) Complement associated microvascular injury and thrombosis in the pathogenesis of severe COVID-19 infection: A report of five cases. Transl Res 220, 1-13.
[39] Zhang Y, Xiao M, Zhang S, Xia P, Cao W, Jiang W, Chen H, Ding X, Zhao H, Zhang H, Wang C, Zhao J, Sun X, Tian R, Wu W, Wu D, Ma J, Chen Y, Zhang D, Xie J, Yan X, Zhou X, Liu Z, Wang J, Du B, Qin Y, Gao P, Qin X, Xu Y, Zhang W, Li T, Zhang F, Zhao Y, Li Y, Zhang S (2020) Coagulopathy and antiphospholipid antibodies in patients with Covid-19. N Engl J Med 382, e38.

[40] Su L, Ma X, Yu H, Zhang Z, Bian P, Han Y, Sun J, Liu Y, Yang C, Geng J, Zhang Z, Gai Z (2020) The different clinical characteristics of corona virus disease cases between children and their families in China - the character of children with COVID-19. Emerg Microbes Infect 9 , 707-713.

[41] Wei M, Yuan J, Liu Y, Fu T, Yu X, Zhang ZJ (2020) Novel coronavirus infection in hospitalized infants under 1 year of age in China. JAMA 323, 1313-1314.

[42] CDC COVID-19 Response Team (2020) Coronavirus Disease 2019 in Children - United States, February 12-April 2, 2020. MMWR Morb Mortal Wkly Rep 69, 422-426.

[43] Verity R, Okell LC, Dorigatti I, Winskill P, Whittaker C, Imai N, Cuomo-Dannenburg G, Thompson H, Walker PGT, Fu H, Dighe A, Griffin JT, Baguelin M, Bhatia S, Boonyasiri A, Cori A, Cucunubá Z, FitzJohn R, Gaythorpe K, Green W, Hamlet A, Hinsley W, Laydon D, NedjatiGilani G, Riley S, van Elsland S, Volz E, Wang H, Wang Y, Xi X, Donnelly CA, Ghani AC, Ferguson NM (2020) Estimates of the severity of coronavirus disease 2019: A model-based analysis. Lancet Infect Dis 20, 669-677.

[44] Huang Y, Tu M, Wang S, Chen S, Zhou W, Chen D, Zhou L, Wang M, Zhao Y, Zeng W, Huang Q, Xu Hb, Liu Z, Guo L (2020) Clinical characteristics of laboratory confirmed positive cases of SARS-CoV-2 infection in Wuhan, China: A retrospective single center analysis. Travel Med Infect Dis, doi: 10.1016/j.tmaid.2020.101606

[45] Aggarwal S, Garcia-Telles N, Aggarwal G, Lavie C, Lippi G, Henry BM (2020) Clinical features, laboratory characteristics, and outcomes of patients hospitalized with coronavirus disease 2019 (COVID-19): Early report from the United States. Diagnosis 7, 91-96.

[46] Lai C-C, Liu YH, Wang C-Y, Wang Y-H, Hsueh S-C, Yen M-Y, Ko W-C, Hsueh P-R (2020) Asymptomatic carrier state, acute respiratory disease, and pneumonia due to severe acute respiratory syndrome coronavirus 2 (SARS-CoV-2): Facts and myths. J Microbiol Immunol 53, 404-412.

[47] Zhang JJ, Dong X, Cao YY, Yuan YD, Yang YB, Yan YQ, Akdis CA, Gao YD (2020) Clinical characteristics of 140 patients infected with SARS-CoV-2 in Wuhan, China. Allergy 75, 1730-1741.

[48] Cascella M, Rajnik M, Cuomo A, Dulebohn SC, Di Napoli R (2020) Features, evaluation and treatment coronavirus (COVID-19) StatPearls Publishing, https://www.ncbi.nlm.nih.gov/books/NBK554776/.

[49] Liu K, Chen Y, Lin R, Han K (2020) Clinical features of COVID-19 in elderly patients: A comparison with young and middle-aged patients. J Infect 80, e14-e18.

[50] Xu Y-H, Dong J-H, An W-M, Lv X-Y, Yin X-P, Zhang J-Z, Dong L, Ma X, Zhang H-J, Gao B-L (2020) Clinical and computed tomographic imaging features of novel coronavirus pneumonia caused by SARS-CoV-2. J Infect 80, 394-400.

[51] Olofsson S, Kumlin U, Dimock K, Arnberg N (2005) Avian influenza and sialic acid receptors: More than meets the eye? Lancet Infect Dis 5, 184-188. 
[52] Hu K, Patel J, Patel BC (2020) Ophthalmic manifestations of coronavirus (COVID-19). StatPearls. StatPearls Publishing, Treasure Island, FL.

[53] Marinho PM, Marcos AAA, Romano AC, Nascimento H, Belfort R (2020) Retinal findings in patients with COVID19. Lancet 395, 1610.

[54] Dumitrascu OM, Volod O, Bose S, Wang Y, Biousse V, Lyden PD (2020) Acute ophthalmic artery occlusion in a Covid-19 patient on apixaban. J Stroke Cerebrovasc 29, 104982.

[55] Peiris JSM, Yuen KY, Osterhaus ADME, Stöhr K (2003) The severe acute respiratory syndrome. N Engl J Med 349, 2431-2441.

[56] Karimi S, Arabi A, Shahraki T, Safi S (2020) Detection of severe acute respiratory syndrome Coronavirus- 2 in the tears of patients with Coronavirus disease 2019. Eye 34, $1-4$.

[57] Xia J, Tong J, Liu M, Shen Y, Guo D (2020) Evaluation of coronavirus in tears and conjunctival secretions of patients with SARS-CoV-2 infection. J Med Virol 92, 589-594.

[58] Desforges M, Le Coupanec A, Dubeau P, Bourgouin A, Lajoie L, Dubé M, Talbot PJ (2020) Human coronaviruses and other respiratory viruses: Underestimated opportunistic pathogens of the central nervous system? Viruses 12, 14.

[59] Jacomy H, St-Jean JR, Brison E, Marceau G, Desforges M, Talbot PJ (2010) Mutations in the spike glycoprotein of human coronavirus OC43 modulate disease in BALB/c mice from encephalitis to flaccid paralysis and demyelination. J Neurovirol 16, 279-293.

[60] Brison E, Jacomy H, Desforges M, Talbot PJ (2011) Glutamate excitotoxicity is involved in the induction of paralysis in mice after infection by a human coronavirus with a single point mutation in its spike protein. J Virol 85, 1246412473.

[61] Dube M, Le Coupanec A, Wong AHM, Rini JM, Desforges M, Talbot PJ (2018) Axonal transport enables neuron-toneuron propagation of human coronavirus OC43. $J$ Virol 92, 00404-00418.

[62] Stodola JK, Dubois G, LeCoupanec A, Desforges M, Talbot PJ (2018) The OC43 human coronavirus envelope protein is critical for infectious virus production and propagation in neuronal cells and is a determinant of neurovirulence and CNS pathology. Virology 515, 134-149.

[63] Desforges M, Le Coupanec A, Stodola JK, MeessenPinard M, Talbot PJ (2014) Human coronaviruses: Viral and cellular factors involved in neuroinvasiveness and neuropathogenesis. Virus Res 194, 145-158.

[64] Schwerk C, Tenenbaum T, Kim KS, Schroten H (2015) The choroid plexus-a multi-role player during infectious diseases of the CNS. Front Cell Neurosci 9, 80.

[65] Mori I, (2015) Transolfactory neuroinvasion by viruses threatens the human brain. Acta Virol 59, 338-349.

[66] Bryche B, Frétaud M, Saint-Albin Deliot A, Galloux M, Sedano L, Langevin C, Descamps D, Rameix-Welti M-A, Eléouët J-F, Le Goffic R, Meunier N (2019) Respiratory syncytial virus tropism for olfactory sensory neurons in mice. J Neurochem, doi: 10.1111/jnc. 14936

[67] Dups J, Middelton D, Yamada M, Monaghan P, Long F, Robinson R, Marsh GA, Wang L-F (2012) A new model for hendra virus encephalitis in the mouse. PLoS One 7, e40308.

[68] Bohmwald K, Galvez NMS, Rios M, Kalergis AM (1018) Neurologic alterations due to respiratory virus infections. Front Cell Neurosci 12, 386.
[69] Driessen AK, Farrell MJ, Mazzone SB, McGovern AE (2016) Multiple neural circuits mediating airway sensations: Recent advances in the neurobiology of the urge-to-cough. Respir Physiol Neurobiol 226, 115-120.

[70] Audrit KJ, Delventhal L, Aydin O, Nassenstein C (2017) The nervous system of airways and its remodeling in inflammatory lung diseases. Cell Tissue Res 367, 571-590.

[71] Park CH, Ishinaka M, Takada A, Kida H, Kimura T, Ochiai K, Umemura T (2002) The invasion routes of Neurovirulent A/Hong Kong/483/97 (H5N1) influenza virus into the central nervous system after respiratory infection in mice. Arch Virol 147, 1425-1436.

[72] Matsuda K, Park CH, Sunden Y, Kimura T, Ochiai K, Kida H, Umemura (2004) The vagus nerve is one route of transneural invasion for intranasally inoculated influenza a virus in mice. Vet Pathol 41, 101-107.

[73] Gu J, Gong E, Zhang B, Zheng J, Gao Z, Zhong Y, Zou W, Zhan J, Wang S, Xie Z, Zhuang H, Wu B, Zhong H, Shao H, Fang W, Gao D, Pei F, Li X, He Z, Xu D, Shi X, Anderson VM, Leong ASY (2005) Multiple organ infection and the pathogenesis of SARS. J Exp Med 202, 415-424.

[74] Raj VS, Osterhaus AD, Fouchier RA, Haagmans BL (2014) MERS: Emergence of a novel human coronavirus. Curr Opin Virol 5, 58-62.

[75] Jevsnik M, Steyer A, Pokorn M, Mrvič T, Grosek Š, Strle F, Lusa L, Petrovec M (2016) The role of human coronaviruses in children hospitalized for acute bronchiolitis, acute gastroenteritis, and febrile seizures: A 2-year prospective study. PLoS One 11, e0155555.

[76] Riski H, Hovi T (1980) Coronavirus infections of man associated with diseases other than the common cold. $J$ Med Virol 6, 259-265.

[77] Gerna G, Passarani N, Battaglia M, Rondanelli EG (1985) Human enteric coronaviruses: Antigenic relatedness to human coronavirus OC43 and possible etiologic role in viral gastroenteritis. J Infect Dis 151, 796-803.

[78] Resta S, Luby JP, Rosenfeld CR, Siegel JD (1985) Isolation and propagation of a human enteric coronavirus. Science 229, 978-981.

[79] Morfopoulou S, Brown JR, Davies EG, Anderson G, Virasami A, Qasim W, Chong WK, Hubank M, Plagnol V, Desforges M, Jaques TS, Talbot PJ, Breuer J (2016) Human coronavirus OC43 associated with fatal encephalitis. N Engl J Med 375, 497-498.

[80] Arbour N, Day R, Newcombe J, Talbot PJ (2000) Neuroinvasion by human respiratory coronaviruses. J Virol 74, 8913-8921.

[81] Cristallo A, Gambaro F, Biamonti G, Ferrante P, Battaglia M, Cereda PM (1997) Human coronavirus polyadenylated RNA sequences in cerebrospinal fluid from multiple sclerosis patients. New Microbiol 20, 105-114.

[82] Fazzini E, Fleming J, Fahn S (1992) Cerebrospinal fluid antibodies to coronavirus in patients with Parkinson's disease. Mov Disord 7, 153-158.

[83] Stewart JN, Mounir S, Talbot PJ (1992) Human coronavirus gene expression in the brains of multiple sclerosis patients. Virology 191, 502-505.

[84] Bookstaver PB, Mohorn PL, Shah A, Tesh LD, Quidley AM, Kothari R, Bland CM, Weissman S (2017) Management of viral central nervous system infections: A primer for clinicians. J Cent Nerv Syst Dis 9, 1179573517703342.

[85] Kennedy PG (2004) Viral encephalitis: Causes, differential diagnosis, and management. J Neurol Neurosurg Psychiatry 75, i10-i15. 
[86] Kennedy PG (2005) Viral encephalitis. J Neurol 252, 268272.

[87] Costa BKD, Sato DK (2020) Viral encephalitis: A practical review on diagnostic approach and treatment. J Pediatr 96, 12-19.

[88] Solomon IH, Normandin E, Bhattacharyya S, Mukerji SS, Keller K, Ali AS, Adams G, Hornick JL, Padera RF, Sabeti P (2020) Neuropathological features of Covid-19. N Engl J Med, doi: 10.1056/NEJMc2019373

[89] Varatharaj A, Thomas N, Ellul MA, Davies NWS, Pollak TA, Tenorio EL, Sultan M, Easton A, Breen G, Zandi M, Coles JP, Manji H, Al-Shahi Salman R, Menon DK, Nicholson TR, Benjamin LA, Carson A, Smith C, Turner MR, Solomon T, Kneen R, Pett SL, Galea I, Thomas RH, Michael BD; CoroNerve Study Group (2020) Neurological and neuropsychiatric complications of COVID-19 in 153 patients: A UK-wide surveillance study. Lancet Psychiatry, doi: 10.1016/S2215-0366(20)30287-X

[90] Mao L, Jin H, Wang M, Hu Y, Chen S, He Q, Chang J, Hong C, Zhou Y, Wang D, Miao X, Li Y, Hu B (2020) Neurologic manifestations of hospitalized patients with coronavirus disease 2019 in Wuhan, China. JAMA Neurol 77, 683-690.

[91] Brann DH, Tsukahara T, Weinreb C, Lipovsek M, Van den Berge K, Gong B, Chance R, Macaulay IC, Chou H-J, Fletcher RB, Das D, Street K, de Bezieux HR, Choi Y-G, Risso D, Dudoit S, Purdom E, Mill J, Hachem RA, Matsunami H, Logan DW, Goldstein BJ, Grubb MS, Ngai J, Datta SR (2020) Non-neuronal expression of SARS-CoV2 entry genes in the olfactory system suggests mechanisms underlying COVID-19-associated anosmia. Sci $A d v \mathbf{6}$, eabc5801.

[92] Lechien JR, Chiesa-Estomba CM, De Siati DR, Horoi M, Le Bon SD, Rodriguez A, Dequanter D, Blecic S, El Afia F, Distinguin L, Chekkoury-Idrissi Y, Hans S, Delgado IL, Calvo-Henriquez C, Lavigne P, Falanga C, Barillari MR, Cammaroto G, Khalife M, Leich P, Souchay C, Rossi C, Journe F, Hsieh J, Edjlali M, Carlier R, Ris L, Lovato A, De Filippis C, Coppee F, Fakhry N, Ayad T, Saussez S (2020) Olfactory and gustatory dysfunctions as a clinical presentation of mild-to-moderate forms of the coronavirus disease (COVID-19): A multicenter European study. Eur Arch Otorhinolaryngol 277, 2251-2261.

[93] Helms J, Kremer S, Merdji H, Clere-Jehl R, Schenck M, Kummerlen C, Collange O, Boulay C, Fafi-Kremer S, Ohana M, Anheim M, Meziani F (2020) Neurologic features in severe SARS-CoV-2 infection. N Engl J Med 328, 2268-2270.

[94] Oxley TJ, Mocco J, Majidi S, Kellner CP, Shoirah H, Singh IP, De Leacy RA, Shigematsu T, Ladner TR, Yaeger KA, Skliut M, Weinberger J, Dangayach NS, Bederson JB, Tuhrim S, Fifi JT (2020) Large-vessel stroke as a presenting feature of Covid-19 in the young. N Engl J Med 382, e60.

[95] Cariou B, Hadjadj S, Wargny M, Pichelin M, Al-Salameh A, Allix I, Amadou C, Arnault G, Baudoux F, Bauduceau B, Borot S, Bourgeon-Ghittori M, Bourron O, Boutoille D, Cazenave-Roblot F, Chaumeil C, Cosson E, Coudol S, Darmon P, Disse E, Ducet-Boiffard A, Gaborit B, Joubert M, Kerlan V, Laviolle B, Marchand L, Meyer L, Potier L, Prevost G, Riveline JP, Robert R, Saulnier PJ, Sultan A, Thébaut JF, Thivolet C, Tramunt B, Vatier C, Roussel R, Gautier JF, Gourdy P; CORONADO investigators (2020) Phenotypic characteristics and prognosis of inpa- tients with COVID-19 and diabetes: The CORONADO study. Diabetologia 63, 1500-1515.

[96] Trejo Gabriel y Galán JM (2020) Stroke as a complication and prognostic factor of COVID-19. Neurología 35, 318322.

[97] Román GC, Spencer PS, Reis J, Buguet A, El Alaoui Faris M, Katrak SM, Láinez M, Medina MT, Meshram C, Mizusawa H, Öztürk S, Wasay M (2020) The neurology of COVID-19 revisited: A proposal from the Environmental Neurology Specialty Group of the World Federation of Neurology to implement international neurological registries. J Neurol Sci 414, 116884.

[98] Klok FA, Kruip MJHA, van der Meer NJM, Arbous MS, Gommers DAMPJ, Kant KM, Kaptein FHJ, van Paassen J, Stals MAM, Huisman MV, Endeman H (2020) Incidence of thrombotic complications in critically ill ICU patients with COVID-19. Thromb Res 191, 145-147.

[99] Beyrouti R, Adams ME, Benjamin L, Cohen H, Farmer SF, Goh YY, Humphries F, Jäger HR, Losseff NA, Perry RJ, Shah S, Simister RJ, Turner D, Chandratheva A, Werring DJ (2020) Characteristics of ischaemic stroke associated with COVID-19. J Neurol Neurosurg Psychiatry 91, 889.

[100] Lodigiani C, Iapichino G, Carenzo L, Cecconi M, Ferrazzi P, Sebastian T, Kucher N, Studt J-D, Sacco C, Alexia B, Sandri MT, Barco S (2020) Venous and arterial thromboembolic complications in COVID-19 patients admitted to an academic hospital in Milan, Italy. Thromb Res 191, 9-14.

[101] Al Saiegh F, Ghosh R, Leibold A, Avery MB, Schmidt RF, Theofanis T, Mouchtouris N, Philipp L, Peiper SC, Wang Z-X, Rincon F, Tjoumakaris SI, Jabbour P, Rosenwasser RH, Gooch MR (2020) Status of SARS-CoV-2 in cerebrospinal fluid of patients with COVID-19 and stroke. $J$ Neurol Neurosurg Psychiatry 91, 846-848.

[102] Gutiérrez-Ortiz C, Méndez A, Rodrigo-Rey S, San PedroMurillo E, Bermejo-Guerrero L, Gordo-Mañas R, de Aragón-Gómez F, Benito-León J (2020) Miller Fisher Syndrome and polyneuritis cranialis in COVID-19. Neurology 95, e601-e605.

[103] Ye M, Ren Y, Lv T (2020) Encephalitis as a clinical manifestation of COVID-19. Brain Behav Immun 88, 945-946.

[104] Zhou L, Zhang M, Wang J, Gao J (2020) Sars-Cov-2: Underestimated damage to nervous system. Travel Med Infect Dis, doi: 10.1016/j.tmaid.2020.101642

[105] Moriguchi T, Harii N, Goto J, Harada D, Sugawara H, Takamino J, Ueno M, Sakata H, Kondo K, Myose N, Nakao A, Takeda M, Haro H, Inoue O, Suzuki-Inoue K, Kubokawa K, Ogihara S, Sasaki T, Kinouchi H, Kojin H, Ito M, Onishi H, Shimizu T, Sasaki Y, Enomoto N, Ishihara H, Furuya S, Yamamoto T, Shimada S (2020) A first case of meningitis/encephalitis associated with SARS-Coronavirus-2. Int J Infect Dis 94, 55-58.

[106] Politi LS, Salsano E, Grimaldi M (2020) Magnetic resonance imaging alteration of the brain in a patient with coronavirus disease 2019 (COVID-19) and anosmia. JAMA Neurol, doi: 10.1001/jamaneurol.2020.2125

[107] Bagheri SH, Asghari A, Farhadi M, Shamshiri AR, Kabir A, Kamrava SK, Jalessi M, Mohebbi A, Alizadeh R, Honarmand AA, Ghalehbaghi B, Salimi A, Dehghani Firouzabadi F (2020) Coincidence of COVID-19 epidemic and olfactory dysfunction outbreak in Iran. MJIRI 34, 446452.

[108] Yi Y, Lagniton PNP, Ye S, Li E, Xu R-H (2020) COVID19: What has been learned and to be learned about 
the novel coronavirus disease. Int J Biol Sci 16, 17531766.

[109] Chen G, Wu D, Guo W, Cao Y, Huang D, Wang H, Wang T, Zhang X, Chen H, Yu H, Zhang X, Zhang M, Wu S, Song J, Chen T, Han M, Li S, Luo X, Zhao J, Ning Q (2020) Clinical and immunological features of severe and moderate coronavirus disease 2019. J Clin Invest 130, 2620-2629.

[110] Simpson S, Kay FU, Abbara S, Bhalla S, Chung JH, Chung M, Henry TS, Kanne JP, Kligerman S, Ko JP, Litt H (2020) Radiological Society of North America Expert Consensus Statement on Reporting Chest CT Findings Related to COVID-19. Endorsed by the Society of Thoracic Radiology, the American College of Radiology, and RSNA. Radiol Cardiothorac Imaging 2, e200152.

[111] Cassaniti I, Novazzi F, Giardina F, Salinaro F, Sachs M, Perlini S, Bruno R, Mojoli F, Baldanti F, Members of the San Matteo Pavia COVID-19 Task Force (2020) Performance of VivaDiag COVID-19 IgM/IgG Rapid Test is inadequate for diagnosis of COVID-19 in acute patients referring to emergency room department. J Med Virol, doi: 10.1002/jmv.25800

[112] Ye Z, Zhang Y, Wang Y, Huang Z, Song B (2020) Chest CT manifestations of new coronavirus disease 2019 (COVID19): A pictorial review. Eur Radiol 30, 4381-4389.

[113] Zhang C, Shi L, Wang F-S (2020) Liver injury in COVID19: Management and challenges. Lancet Gastroenterol 5, 428-430.

[114] Herold T, Jurinovic V, Arnreich C, Lipworth BJ, Hellmuth JC, Bergwelt-Baildon M von, Klein M, Weinberger T (2020) Elevated levels of IL-6 and CRP predict the need for mechanical ventilation in COVID-19. J Allergy Clin Immun 146, 128-136.e4.

[115] Han H, Ma Q, Li C, Liu R, Zhao L, Wang W, Zhang P, Liu X, Gao G, Liu F, Jiang Y, Cheng X, Zhu C, Xia Y (2020) Profiling serum cytokines in COVID-19 patients reveals IL-6 and IL-10 are disease severity predictors. Emerg Microbes Infect 9, 1123-1130.

[116] Liu F, Li L, Xu M, Wu J, Luo D, Zhu Y, Li B, Song X, Zhou $X$ (2020) Prognostic value of interleukin-6, C-reactive protein, and procalcitonin in patients with COVID-19. J Clin Virol 127, 104370.

[117] CDC (2020) Clinical Specimens: Novel Coronavirus (2019-nCoV). https://www.cdc.gov/coronavirus/2019nCoV/lab/guidelines-clinical-specimens.html, Last updated 8 July 2020, Accessed 30 July 2020.

[118] Al-Tawfiq JA, Memish ZA (2020) Diagnosis of SARSCoV-2 infection based on CT scan vs RT-PCR: Reflecting on experience from MERS-CoV. J Hosp Infect 105, 154155.

[119] Japan ECMOnet for COVID-19 (2020) Nationwide system to centralize decisions around ECMO use for severe COVID-19 pneumonia in Japan (Special Correspondence). J Intensive Care 8, 29.

[120] Ledford H (2020) Coronavirus breakthrough: Dexamethasone is first drug shown to save lives. Nature 582, 469-469.

[121] Fox SE, Akmatbekoy A, Harbert JL, Li G, Brown JQ, Vander Heide RS (2020) Pulmonary and cardiac pathology in African American patients with COVID-19: An autopsy series from New Orleans. Lancet Respir Med 8, 681-686.

[122] Motta JK, Ogunnaike RO, Shah R, Stroever S, Cedeno HV, Thapa SK, Chronakos JJ, Jimenez EJ, Petrini J, Hegde A (2020) Clinical outcomes with the use of prophylactic versus therapeutic anticoagulation in COVID-19. medRxiv, doi: 10.1101/2020.07.20.20147769
[123] Guérin C, Reignier J, Richard J-C, Beuret P, Gacouin A, Boulain T, Mercier E, Badet M, Mercat A, Baudin O, Clavel M, Chatellier D, Jaber S, Rosselli S, Mancebo J, Sirodot M, Hilbert G, Bengler C, Richecoeur J, Gainnier M, Bayle F, Bourdin G, Leray V, Girard R, Baboi L, Ayzac L, PROSEVA Study Group (2013) Prone positioning in severe acute respiratory distress syndrome. $N$ Engl J Med 368, 2159-2168.

[124] Gurwitz D (2020) Angiotensin receptor blockers as tentative SARS-CoV-2 therapeutics. Drug Dev Res 81, 537-540.

[125] Ko W-C, Rolain J-M, Lee N-Y, Chen P-L, Huang C-T, Lee P-I, Hsueh P-R (2020) Arguments in favour of remdesivir for treating SARS-CoV-2 infections. Int J Antimicrob Agents 55, 105933.

[126] Guo Y-R, Cao Q-D, Hong Z-S, Tan Y-Y, Chen S-D, Jin H-J, Tan K-S, Wang D-Y, Yan Y (2020) The origin, transmission and clinical therapies on coronavirus disease 2019 (COVID-19) outbreak - an update on the status. Mil Med Res 7, 11.

[127] Hendaus MA (2020) Remdesivir in the treatment of coronavirus disease 2019 (COVID-19): A simplified summary. J Biomol Struct Dyn, doi: 10.1080/07391102.2020.176 7691

[128] Goldman JD, Lye DCB, Hui DS, Marks KM, Bruno R, Montejano R, Spinner CD, Galli M, Ahn M-Y, Nahass RG, Chen Y-S, SenGupta D, Hyland RH, Osinusi AO, Cao H, Blair C, Wei X, Gaggar A, Brainard DM, Towner WJ, Muñoz J, Mullane KM, Marty FM, Tashima KT, Diaz G, Subramanian A (2020) Remdesivir for 5 or 10 days in patients with severe Covid-19. N Engl J Med, doi: 10.1056/NEJMoa2015301

[129] Roback JD, Guarner J (2020) Convalescent plasma to treat COVID-19: Possibilities and challenges. JAMA 323, 15611562.

[130] Shanmugaraj B, Siriwattananon K, Wangkanont K, Phoolcharoen W (2020) Perspectives on monoclonal antibody therapy as potential therapeutic intervention for Coronavirus disease-19 (COVID-19). Asian Pac J Allergy Immunol 38, 10-18.

[131] Gautret P, Lagier J-C, Parola P, Hoang VT, Meddeb L, Mailhe M, Doudier B, Courjon J, Giordanengo V, Vieira VE, Tissot Dupont H, Honoré S, Colson P, Chabrière E, La Scola B, Rolain J-M, Brouqui P, Raoult D (2020) Hydroxychloroquine and azithromycin as a treatment of COVID-19: Results of an open-label non-randomized clinical trial. Int J Antimicrob Agents 56, 105949.

[132] Liu J, Cao R, Xu M, Wang X, Zhang H, Hu H, Li Y, Hu Z, Zhong W, Wang M (2020) Hydroxychloroquine, a less toxic derivative of chloroquine, is effective in inhibiting SARS-CoV-2 infection in vitro. Cell Discov 6, 16.

[133] Mehra MR, Desai SS, Ruschitzka F, Patel AN (2020) Hydroxychloroquine or chloroquine with or without a macrolide for treatment of COVID-19: A multinational registry analysis. Lancet, doi: 10.1016/S01406736(20)31180-6

[134] Mehra MR, Ruschitzka F, Patel AN (2020) Retraction-Hydroxychloroquine or chloroquine with or without a macrolide for treatment of COVID-19: A multinational registry analysis. Lancet 395, 1820.

[135] Arshad S, Kilgore P, Chaudhry ZS, Jacobsen G, Wang DD, Huitsing K, Brar I, Alangaden GJ, Ramesh MS, McKinnon JE, O'Neill W, Zervos M, Nauriyal V, Hamed AA, Nadeem O, Swiderek J, Godfrey A, Jennings J, GardnerGray J, Ackerman AM, Lezotte J, Ruhala J, Fadel R, 
Vahia A, Gudipati S, Parraga T, Shallal A, Maki G, Tariq Z, Suleyman G, Yared N, Herc E, Williams J, Lanfranco OA, Bhargava P, Reyes K (2020) Treatment with hydroxychloroquine, azithromycin, and combination in patients hospitalized with COVID-19. Int J Infect Dis $\mathbf{9 7 ,}$ 396-403.

[136] Kim E, Erdos G, Huang S, Kenniston TW, Balmert SC, Carey CD, Raj VS, Epperly MW, Klimstra WB, Haagmans BL, Korkmaz E, Falo LD, Gambotto A (2020) Microneedle array delivered recombinant coronavirus vaccines: Immunogenicity and rapid translational development. EBioMedicine 55, 102743.

[137] Metcalf CJE, Ferrari M, Graham AL, Grenfell BT (2015) Understanding herd immunity. Trends Immunol 36, 753755.

[138] Zhang B, Liu S, Dong Y, Zhang L, Zhong Q, Zou Y, Zhang $S$ (2020) Positive rectal swabs in young patients recovered from coronavirus disease 2019 (COVID-19). J Infection 81, e49-e52.

[139] Trouillet-Assant S, Viel S, Gaymard A, Pons S, Richard JC, Perret M, Villard M, Brengel-Pesce K, Lina B, Mezidi M, Bitker L, Belot A, Mouton W, Oriol G, Compagnon C, Generenaz L, Cheynet V, Ader F, Becker A, Benech N, Chauvelot P, Chidiac C, Conrad A, Ferry T, Miailhes P, Perpoint T, Perry M, Pouderoux C, Roux S, Triffault-Fillit C, Valour F, Hodane Y, Chauvelot L, Chabert P, Provoost J, David G, Folliet L, Lecam P, Billaud G, Bouscambert M, Escuret V, Frobert E, Bal A, Destras G, Josset L, Morfin F, Munier C, Valette M, Venet F, Garnier L, Pescarmona R, Lombard C, Walzer T (2020) Type I IFN immunoprofiling in COVID-19 patients. J Allergy Clin Immun 146, 206208.e2.

[140] Wu X, Fu B, Chen L, Feng Y (2020) Serological tests facilitate identification of asymptomatic SARSCoV-2 infection in Wuhan, China. J Med Virol, doi: 10.1002/jmv.25904

[141] Market Watch, Lucking L (2020) Coronavirus antibodies found in $21 \%$ of New Yorkers in early testingMarketWatch, https://www.marketwatch.com/story/ early-antibody-tests-find-21-of-new-yorkers-have-hadcovid-19, Last updated 23 April 2020, Accessed 23 April 2020.

[142] Mayo Clinic, Herd immunity and COVID-19 (coronavirus): What you need to know, (2020) https:// www.mayoclinic.org/diseases-conditions/coronavirus/indepth/herd-immunity-and-coronavirus/art-20486808, Last updated 6 June 2020, Accessed 4 August 2020.

[143] Santos-Pinto L, Mata J, The Problems with the "Natural" Herd Immunity Solution to the Covid-19 Epidemic, (2020) Viral, https://wp.unil.ch/viral/the-problems-withthe-natural-herd-immunity-solution-to-the-covid-19epidemic/ Last Updated 11 May 2020, Accessed 4 August 2020.

[144] D'Souza G, Dowdy D (2020) What is herd immunity and how can we achieve it with COVID-19? Johns Hopkins, https://www.jhsph.edu/covid-19/articles/achievingherd-immunity-with-covid19.html, Posted 10 April 2020, Accessed 10 April 2020.

[145] Flint SJ, Racaniello VR, Rall GF, Skalka AM, Enquist LW (2015) Principles of virology, ASM Press, Washington, DC.

[146] Wang F, Nie J, Wang H, Zhao Q, Xiong Y, Deng L, Song S, Ma Z, Mo P, Zhang Y (2020) Characteristics of peripheral lymphocyte subset alteration in COVID-19 pneumonia. $J$ Infect Dis 221, 1762-1769.
[147] Lurie N, Saville M, Hatchett R, Halton J (2020) Developing Covid-19 vaccines at pandemic speed. $N$ Engl J Med 382, 1969-1973.

[148] Knapp A (2020) FDA Green-Lights Clinical Trial For COVID-19 Coronavirus Treatment That 'Flattens The Curve' In Patients, Forbes, https://www.forbes.com/ sites/alexknapp/2020/04/02/fda-gives-green-light-to-testa-treatment-against-covid-19-coronavirus-that-flattensthe-curve-in-patients/\#72a75af04b57, Posted 2 April 2020, Accessed 2 April 2020.

[149] Clinicaltrials.gov, NK Cells Treatment for COVID-19. https://clinicaltrials.gov/ct2/show/NCT04280224, Last updated 14 April 2020, Accessed 29 July 2020.

[150] Clinicaltrials.gov, Phase I/II Study of Universal Offthe-shelf NKG2D-ACE2 CAR-NK Cells for Therapy of COVID-19. https://clinicaltrials.gov/ct2/show/ NCT04324996, Last updated 27 March 2020, Accessed 6 July 2020 .

[151] Sidhu K (2020) The cytokine storm in COVID-19 patients amenable to stem cell treatment: A perspective. Stem Cells Regen Med 4, 1-4.

[152] Braun J, Loyal L, Frentsch M, Wendisch D, Georg P, Kurth F, Hippenstiel S, Dingeldey M, Kruse B, Fauchere F, Baysal E, Mangold M, Henze L, Lauster R, Mall MA, Beyer K, Röhmel J, Voigt S, Schmitz J, Miltenyi S, Demuth I, Müller MA, Hocke A, Witzenrath M, Suttorp N, Kern F, Reimer U, Wenschuh H, Drosten C, Corman VM, Giesecke-Thiel C, Sander LE, Thiel A (2020) SARSCoV-2-reactive $\mathrm{T}$ cells in healthy donors and patients with COVID-19. Nature, doi: 10.1038/s41586-0202598-9

[153] Singh S, Chakravarty T, Chen P, Akhmerov A, Falk J, Friedman O, Zaman T, Ebinger JE, Gheorghiu M, Marbán L, Marbán E, Makkar RR (2020) Allogeneic cardiosphere-derived cells (CAP-1002) in critically ill COVID-19 patients: Compassionate-use case series. Basic Res Cardiol 115, 36.

[154] Praveen D, Puvvada RC, M VA (2020) Janus kinase inhibitor baricitinib is not an ideal option for management of COVID-19. Int J Antimicrob Agents 55, 105967.

[155] Yang N, Shen H-M (2020) Targeting the endocytic pathway and autophagy process as a novel therapeutic strategy in COVID-19. Int J Biol Sci 16, 1724-1731.

[156] Hu TY, Frieman M, Wolfram J (2020) Insights from nanomedicine into chloroquine efficacy against COVID19. Nat Nanotechnol 15, 247-249.

[157] Lombardo D, Kiselev MA, Caccamo MT (2019) Smart nanoparticles for drug delivery application: Development of versatile nanocarrier platforms in biotechnology and nanomedicine. J Nanomater 2019, 1-26.

[158] Kaushik A, Jayant RD, Nair M (2018) Nanomedicine for neuroHIV/AIDS management. Nanomedicine-UK 13, 669-673.

[159] Kaushik A, Jayant RD, Bhardwaj V, Nair M (2018) Personalized nanomedicine for CNS diseases. Drug Discov Today 23, 1007-1015.

[160] Nair M, Jayant RD, Kaushik A, Sagar V (2016) Getting into the brain: Potential of nanotechnology in the management of NeuroAIDS. Adv Drug Deliv Rev 103, 202-217.

[161] Kaushik A, Rodriguez J, Rothen D, Bhardwaj V, Jayant RD, Pattany P, Fuentes B, Chand H, Kolishetti N, El-Hage N, Khalili K, Kenyon NS, Nair M (2019) MRI-guided, noninvasive delivery of magneto-electric drug nanocarriers to the brain in a nonhuman primate. ACS Appl Bio Mater 2, 4826-4836. 
[162] Kaushik A (2019) Biomedical nanotechnology related grand challenges and perspectives. Front Nanotechnol 1,1 .

[163] Azimi P, Keshavarz Z, Cedeno Laurent JG, Stephens BR, Allen JG (2020) Mechanistic transmission modeling of COVID-19 on the Diamond Princess Cruise Ship demonstrates the importance of aerosol transmission. medRxiv, doi: https://doi.org/10.1101/2020.07.13.20153049

[164] Ereth MH, Hess DH, Driscoll A, Hernandez M, Stamatatos F (2020) Particle control reduces fine and ultrafine particles greater than HEPA filtration in live operating rooms and kills biologic warfare surrogate, Am J Infect Control 48, 777-780.

[165] Balli N, Kara E, Demirkan K (2020) The another side of COVID-19 in Alzheimer's disease patients: Drug-drug interactions. Int J Clin Pract, doi: 10.1111/ijcp.13596

[166] Bishara D, Kalafatis C, Taylor D (2020) Emerging and experimental treatments for COVID-19 and drug interactions with psychotropic agents. Ther Adv Psychopharmacol 10, 2045125320935306.

[167] Calati R, Ferrari C, Brittner M, Oasi O, Olié E, Carvalho AF, Courtet P (2019) Suicidal thoughts and behaviors and social isolation: A narrative review of the literature. $J$ Affect Disord 245, 653-667.

[168] Lee SA, Ju YJ, Han K-T, Choi JW, Yoon HJ, Park E-C (2017) The association between loss of work ability and depression: A focus on employment status. Int Arch Occup Environ Health 90, 109-116.

[169] Navarro-Abal Y, Climent-Rodríguez JA, López-López MJ, Gómez-Salgado J (2018) Psychological coping with job loss. Empirical study to contribute to the development of unemployed people. Int J Environ Res Public Health $15,1787$.

[170] Airagnes G, Lemogne C, Meneton P, Plessz M, Goldberg M, Hoertel N, Roquelaure Y, Limosin F, Zins M (2019) Alcohol, tobacco and cannabis use are associated with job loss at follow-up: Findings from the CONSTANCES cohort. PLoS One 14, e0222361.

[171] de Goeij MCM, Bruggink J-W, Otten F, Kunst AE (2017) Harmful drinking after job loss: A stronger association during the post-2008 economic crisis? Int J Public Health 62, 563-572.

[172] Usher K, Bhullar N, Durkin J, Gyamfi N, Jackson D (2020) Family violence and COVID-19: Increased vulnerability and reduced options for support. Int J Mental Health Nurs 29, 549-552.

[173] Matthews T, Danese A, Wertz J, Odgers CL, Ambler A, Moffitt TE, Arseneault L (2016) Social isolation, loneliness and depression in young adulthood: A behavioural genetic analysis. Soc Psychiatry Psychiatr Epidemiol 51, 339-348.

[174] Hawryluck L, Gold WL, Robinson S, Pogorski S, Galea S, Styra R (2004) SARS control and psychological effects of quarantine, Toronto, Canada. Emerg Infect Dis 10, 12061212.

[175] Reynolds DL, Garay JR, Deamond SL, Moran MK, Gold W, Styra R (2008) Understanding, compliance and psychological impact of the SARS quarantine experience. Epidemiol Infect 136, 997-1007.

[176] Keita MM, Taverne B, Sy Savané S, March L, Doukoure M, Sow MS, Touré A, Etard JF, Barry M, Delaporte E, Barry M, Cissé M, Diallo MS, Diallo SB, Kassé D, Magassouba NF, Sow MS, Savané I, Koivogui L, Ayouba A, Delaporte E, Desclaux A, Etard JF, Granouillac B, Izard S, Keita AK, Kpamou C, Leroy S, March L, Msellati P,
Peeters M, Taverne B, Touré A, Baize S, Abel L, Delmas C, Etienne C, Lacabaratz C, Lévy-Marchal C, Lévy Y, Raoul H, the PostEboGui Study Group (2017) Depressive symptoms among survivors of Ebola virus disease in Conakry (Guinea): Preliminary results of the PostEboGui cohort. BMC Psychiatry 17, 127.

[177] Etard J-F, Sow MS, Leroy S, Touré A, Taverne B, Keita AK, Msellati P, Magassouba N, Baize S, Raoul H, Izard S, Kpamou C, March L, Savane I, Barry M, Delaporte E, Postebogui Study Group (2017) Multidisciplinary assessment of post-Ebola sequelae in Guinea (Postebogui): An observational cohort study. Lancet Infect Dis 17, 545-552.

[178] Demoro G, Damico V, Murano L, Bolgeo T, D'Alessandro A, Dal Molin A (2020) Long-term consequences in survivors of critical illness. Analysis of incidence and risk factors. Ann Ist Super Sanita 56, 59-65.

[179] Garrouste-Orgeas M, Flahault C, Vinatier I, Rigaud J-P, Thieulot-Rolin N, Mercier E, Rouget A, Grand H, Lesieur O, Tamion F, Hamidfar R, Renault A, Parmentier-Decrucq E, Monseau Y, Argaud L, Bretonnière C, Lautrette A, Badié J, Boulet E, Floccard B, Forceville X, Kipnis E, Soufir L, Valade S, Bige N, Gaffinel A, Hamzaoui O, Simon G, Thirion M, Bouadma L, Large A, Mira J-P, Amdjar-Badidi N, Jourdain M, Jost P-H, Maxime V, Santoli F, Ruckly S, Vioulac C, Leborgne MA, Bellalou L, Fasse L, Misset B, Bailly S, Timsit J-F (2019) Effect of an ICU diary on posttraumatic stress disorder symptoms among patients receiving mechanical ventilation: A randomized clinical trial. JAMA 322, 229-239.

[180] Lai J, Ma S, Wang Y, Cai Z, Hu J, Wei N, Wu J, Du H, Chen T, Li R, Tan H, Kang L, Yao L, Huang M, Wang H, Wang G, Liu Z, Hu S (2020) Factors associated with mental health outcomes among health care workers exposed to coronavirus disease 2019. JAMA Netw Open 3, e203976.

[181] Huang JZ, Han MF, Luo TD, Ren AK, Zhou XP (2020) [Mental health survey of medical staff in a tertiary infectious disease hospital for COVID-19]. Zhonghua Lao Dong Wei Sheng Zhi Ye Bing Za Zhi 38, 192-195.

[182] Huang Y, Zhao N (2020) Generalized anxiety disorder, depressive symptoms and sleep quality during COVID-19 outbreak in China: A web-based cross-sectional survey. Psychiatry Res 288, 112954.

[183] World Health Organization, The rise and rise of interpersonal violence - an unintended impact of the COVID-19 response on families, https://www.euro.who.int/en/healthtopics/Life-stages/pages/news/news/2020/6/the-rise-andrise-of-interpersonal-violence-an-unintended-impact-ofthe-covid-19-response-on-families, Posted 3 June 2020, Accessed 29 June 2020.

[184] Prague Civil Society Centre, What Covid-19 Taught Us about Domestic Violence, https://praguecivilsociety. org/what-covid-19-taught-us-about-domestic-violence/, Accessed 1 July 2020.

[185] Atkins JL, Masoli JA, Delgado J, Pilling LC, Kuo C-LC, Kuchel G, Melzer D (2020) Preexisting comorbidities predicting severe COVID-19 in older adults in the UK Biobank Community Cohort. medRxiv, doi: 10.1101/2020.05.06.20092700

[186] Office for National Statistics (2020) Deaths involving COVID-19 in the care sector, England and Wales: Deaths occurring up to 12 June 2020 and registered up to 20 June 2020 (provisional), Office for National Statistics, https://www.ons.gov.uk/peoplepopulationandcommunity/ birthsdeathsandmarriages/deaths/articles/deathsinvolving covid19inthecaresectorenglandandwales/deathsoccurring 
upto12june2020andregisteredupto20june2020provisional, Posted 3 July 2020, Accessed 9 July 2020.

[187] Soucheray S, CIDRAP, Nursing homes site of $40 \%$ of US COVID-19 deaths (2020) https://www. cidrap.umn.edu/news-perspective/2020/06/nursinghomes-site-40-us-covid-19-deaths Last updated 2 June 2020, Accessed 4 August 2020.

[188] Data CMS, COVID-19 Nursing Home Data (2020), https://data.cms.gov/stories/s/COVID-19-Nursing-

Home-Data/bkwz-xpvg/. Last Updated 19 July 2020, Accessed 4 August 2020.

[189] National Center for Health Statistics (U.S.), ed. (2019) Long-term care providers and services users in the United States, 2015-2016, U.S. Department of Health and Human Services, Centers for Disease Control and Prevention, National Center for Health Statistics, Washington, DC.

[190] Brown EE, Kumar S, Rajji TK, Pallock BG, Mulsant BH (2020) Anticipating and mitigating the impact of the COVID-19 pandemic on Alzheimer's disease and related dementias. Am J Geriatr Psychiatry 28, 712-721.

[191] Barazzoni R, Bischoff SC, Breda J, Wickramasinghe K, Krznaric Z, Nitzan D, Pirlich M, Singer P (2020) ESPEN expert statements and practical guidance for nutritional management of individuals with SARS-CoV-2 infection. Clin Nutr ESPEN 39, 1631-1638.

[192] NNI Global Website, Martindale R, Jayshil P, Enteral Nutrition in the ICU: Covid-19 challenges, https://www. nestlenutrition-institute.org/resources/videos/details/ente ral-nutrition-in-the-icu-covid-19-challenges, Last updated 30 July 2020, Accessed 30 July 2020.

[193] Martindale RG, Berlana D, Boullata JI, Cai W, Calder PC, Deshpande GH, Evans D, Garcia-de-Lorenzo A, Goulet OJ, Li A, Mayer K, Mundi MS, Muscaritoli M, Pradelli L, Rosenthal M, Seo J-M, Waitzberg DL, Klek S (2020) Summary of Proceedings and Expert Consensus Statements from the International Summit "Lipids in Parenteral Nutrition." Jpen Parenter Enter 44, S7-S20.

[194] (2020) Alzheimer's disease research enterprise in the era of COVID-19/SARS-CoV-2. Alzheimers Dement 16, 587588.

[195] Perry G (2020) Alzheimer's disease patients in the crosshairs of COVID-19. J Alzheimers Dis 76, 1.

[196] Wang H, Li T, Barbarino P, Gauthier S, Brodaty H, Molinuevo JL, Xie H, Sun Y, Yu E, Tang Y, Weidner W, Yu $\mathrm{X}$ (2020) Dementia care during COVID-19. Lancet 395, 1190-1191.

[197] Bauer K, Schwarzkopf L, Graessel E, Holle R (2014) A claims data-based comparison of comorbidity in individuals with and without dementia. BMC Geriatrics 14, 10.

[198] Gzil F, Clause-Verdreau AC, Brugeron PE, Hirsch E (2020) Éthique, soin et grand âge pendant l'épidémie de Covid-19. Geriatr Psychol Neuropsychiatr Vieil 18, 151156.

[199] Nutricia Webinar, Wishmeyer P, Van Zanten A, DeWaele E., Strategies for Nutritional Management of COVID-19 Patients post-ICU, https://www.nutriciacongresses.com/ live-webinars/webinar/23/strategies-for-the-nutritionalmanagement-of-covid-19-patients-post-icu/, Last updated 12 May 2020, Accessed 12 May 2020.

[200] Brugliera L, Spina A, Castellazzi P, Cimino P, Tettamanti A, Houdayer E, Arcuri P, Alemanno F, Mortini P, Iannaccone $S$ (2020) Rehabilitation of COVID-19 patients. $J$ Rehabil Med 52, jrm00046.
[201] Sheehy LM (2020) Considerations for postacute rehabilitation for survivors of COVID-19. JMIR Public Health Surveill 6, e19462.

[202] Jin M, Tong Q (2020) Rhabdomyolysis as potential late complication associated with COVID-19. Emerg Infecti Dis 26, 1618-1620.

[203] Poyiadji N, Shahin G, Noujaim D, Stone M, Patel S, Griffith B (2020) COVID-19-associated acute hemorrhagic necrotizing encephalopathy: Imaging features. Radiology 296, E119-E120.

[204] Chen C, Zhou Y, Wang DW (2020) SARS-CoV-2: A potential novel etiology of fulminant myocarditis. Herz 45, 230-232.

[205] Zhou C, Gao C, Xie Y, Xu M (2020) COVID-19 with spontaneous pneumomediastinum. Lancet Infect Dis 20, 510.

[206] Diao B, Wang C, Wang R, Feng Z, Tan Y, Wang H, Wang C, Liu L, Liu Y, Liu Y, Wang G, Yuan Z, Ren L, Wu Y, Chen Y (2020) Human kidney is a target for novel severe acute respiratory syndrome coronavirus 2 (SARSCoV-2) infection, infectious diseases (except HIV/AIDS). medRxiv, doi: 10.1101/2020.03.04.20031120

[207] Law S, Leung AW, Xu C (2020) Is reinfection possible after recovery from COVID-19? Hong Kong Med J 26, 264-265.

[208] Batisse D, Benech N, Botelho-Nevers E, Bouiller K, Collarino R, Conrad A, Gallay L, Goehringer F, Gousseff M, Joseph DC, Lemaignen A, Lescure F-X, Levy B, Mahevas M, Penot P, Pozzetto B, Salmon D, Slama D, Vignier N, Wyplosz B (2020) Clinical recurrences of COVID-19 symptoms after recovery: Viral relapse, reinfection or inflammatory rebound? $J$ Infect, doi: 10.1016/j.jinf.2020.06.073

[209] Oke J, Heneghan C (2020) Global Covid-19 Case Fatality Rates, Global Covid-19 Case Fatality Rates, Centre for Evidence-Based Medicine, CEBM, https://www.cebm.net/covid-19/global-covid-19-casefatality-rates/, Posted 17 March 2020, Accessed 30 July 2020.

[210] AAIC, Flu, Pneumonia Vaccinations Tied to Lower Risk of Alzheimer's Dementia | AAIC 2020 | Alzheimer's Association, https://www.alz.org/aaic/releases_2020/vaccinesdementia-risk.asp, Last updated 27 July 2020; Accessed on 11 August 2020.

[211] Sun JS (2020) Can We Beat SARS-CoV-2? Lessons from Other Coronaviruses, Contagion Live, https://www. contagionlive.com/news/can-we-beat-sarscov2-lessonsfrom-other-coronaviruses, Posted 27 March 2020, Accessed 30 July 2020.

[212] Clinicaltrials.gov, BCG Vaccine for Health Care Workers as Defense Against COVID 19 (BADAS). https://clinicaltrials.gov/ct2/show/NCT04348370, Last updated 27 May 2020, Accessed 6 July 2020.

[213] Clinicaltrials.gov, Bacillus Calmette-guérin Vaccination to Prevent COVID-19 (ACTIVATEII). https://clinicaltrials.gov/ct2/show/NCT04414267, Last updated 14 July 2020, Accessed 29 July 2020.

[214] Clinicaltrials.gov, BCG Vaccination to Protect Healthcare Workers Against COVID-19 (BRACE). https:// clinicaltrials.gov/ct2/show/NCT04327206, Last updated 27 May 2020, Accessed 6 July 2020.

[215] Clinicaltrials.gov, Reducing Health Care Workers Absenteeism in Covid-19 Pandemic Through BCG Vaccine (BCG-CORONA). https://clinicaltrials.gov/ct2/ 
show/NCT04328441, Last updated 29 April 2020, Accessed 6 July 2020.

[216] Clinicaltrials.gov, BCG Vaccination for Healthcare Workers in COVID-19 Pandemic. https://clinicaltrials. gov/ct2/show/NCT04379336, Last updated 7 May 2020, Accessed 6 July 2020.

[217] Clinicaltrials.gov, Study to Assess VPM1002 in Reducing Healthcare Professionals' Absenteeism in COVID-19 Pandemic. https://clinicaltrials.gov/ ct2/show/NCT04387409, Last updated 16 June 2020, Accessed 6 July 2020.

[218] Clinicaltrials.gov, Study to Assess VPM1002 in Reducing Hospital Admissions and/or Severe Respiratory Infectious Diseases in Elderly in COVID-19 Pandemic. https://clinicaltrials.gov/ct2/show/NCT04435379, Last updated 25 June 2020, Accessed 6 July 2020.

[219] Clinicaltrials.gov, Measles Vaccine in HCW (MVCOVID19). https://clinicaltrials.gov/ct2/show/NCT0435 7028, Last updated 18 June 2020, Accessed 6 July 2020.

[220] Clinicaltrials.gov, Investigating a Vaccine Against COVID-19. https://clinicaltrials.gov/ct2/show/NCT044 00838, Last updated 2 July 2020, Accessed 6 July 2020.

[221] Clinicaltrials.gov A Phase II Clinical Trial to Evaluate the Recombinant Vaccine for COVID-19 (Adenovirus Vector) (CTII-nCoV https://clinicaltrials.gov/ ct2/show/NCT04341389, Last updated 18 May 2020, Accessed 6 July 2020.

[222] Clinicaltrials.gov, Dose-Confirmation Study to Evaluate the Safety, Reactogenicity, and Immunogenicity of mRNA-1273 COVID-19 Vaccine in Adults Aged 18 Years and Older. https://clinicaltrials.gov/ct2/show/ NCT04405076, Last updated 18 June 2020, Accessed 6 July 2020.

[223] Clinicaltrials.gov, Safety and Immunogenicity Study of Inactivated Vaccine for Prophylaxis of SARS CoV-2 Infection (COVID-19). https:// clinicaltrials.gov/ct2/show/NCT04352608, Last updated 28 April 2020, Accessed 6 July 2020.

[224] Chinese Clinical Trial Registry, Novel coronavirus (2019-CoV) inactivated vaccine (Vero cell) Phase I/II clinical trial. http://www.chictr.org.cn/showproj. aspx?proj=53003, Last updated 4 May 2020, Accessed 6 July 2020.

[225] Clinicaltrials.gov, Safety and Immunogenicity Study of 2019-nCoV Vaccine (mRNA-1273) for Prophylaxis of SARS-CoV-2 Infection (COVID-19). https://clinicaltrials.gov/ct2/show/NCT04283461, Last updated 29 May 2020, Accessed 6 July 2020.

[226] Clinicaltrials.gov, An Open Study of the Safety, Tolerability and Immunogenicity of the Drug "Gam-COVID-Vac" Vaccine Against COVID-19. https://clinicaltrials.gov/ct2/show/NCT04436471, Last updated 22 June 2020, Accessed 6 July 2020.

[227] Clinicaltrials.gov, An Open Study of the Safety, Tolerability and Immunogenicity of "Gam-COVID-Vac Lyo" Vaccine Against COVID-19. https://clinicaltrials. gov/ct2/show/NCT04437875, Last updated 22 June 2020, Accessed 6 July 2020.

[228] Clinicaltrials.gov, Safety and Immunogenicity Study of GX-19, a COVID-19 Preventive DNA Vaccine in Healthy Adults. https://clinicaltrials.gov/ct2/show/NCT044453 89, Last updated 26 June 2020, Accessed 6 July 2020.

[229] Chinese Clinical Trial Registry, A randomized, doubleblind, placebo parallel-controlled phase I/II clini- cal trial for inactivated Novel Coronavirus Pneumonia vaccine (Vero cells). http://www.chictr.org.cn/show projen.aspx?proj=52227, Last updated 13 March 2020, Accessed 6 July 2020.

[230] Clinicaltrials.gov, Safety, Tolerability and Immunogenicity of INO-4800 for COVID-19 in Healthy Volunteers. https://clinicaltrials.gov/ct2/show/NCT04336410, Last updated 5 June 2020, Accessed 6 July 2020.

[231] Clinicaltrials.gov, Evaluation of the Safety and Immunogenicity of a SARS-CoV-2 rS (COVID-19) Nanoparticle Vaccine With/Without Matrix-M Adjuvant. https://clinicaltrials.gov/ct2/show/NCT04368988, Last updated 27 May 2020, Accessed 6 July 2020.

[232] Clinicaltrials.gov, Safety and Immunogenicity Study of 2019-nCoV Vaccine (mRNA-1273) for Prophylaxis of SARS-CoV-2 Infection (COVID-19). https://clinicaltrials.gov/ct2/show/NCT04283461, Last updated 29 May 2020, Accessed 6 July 2020.

[233] Clinicaltrials.gov, A Study to Evaluate the Safety, Reactogenicity and Immunogenicity of Vaccine CVn$\mathrm{CoV}$ in Healthy Adults. https://clinicaltrials.gov/ct2/ show/NCT04449276, Last updated 1 July 2020, Accessed 6 July 2020.

[234] Clinicaltrials.gov, SCB-2019 as COVID-19 Vaccine. https://clinicaltrials.gov/ct2/show/NCT04405908, Last updated 23 June 2020, Accessed 6 July 2020.

[235] Clinicaltrials.gov, Monovalent Recombinant COVID19 Vaccine (COVAX19). https://clinicaltrials.gov/ct2/show/ NCT04453852, Last updated 1 July 2020, Accessed 6 July 2020.

[236] Clinicaltrials.gov, Tableted COVID-19 Therapeutic Vaccine (COVID-19). https://clinicaltrials.gov/ct2/show/ NCT04380532, Last updated 27 May 2020, Accessed 6 July 2020.

[237] Clinicaltrials.gov, Immunity and Safety of Covid19 Synthetic Minigene Vaccine. https://clinicaltrials. gov/ct2/show/NCT04276896, Last updated 19 March 2020, Accessed 6 July 2020.

[238] Clinicaltrials.gov, A Trial Investigating the Safety and Effects of Four BNT162 Vaccines Against COVID2019 in Healthy Adults. https://clinicaltrials.gov/ct2/ show/NCT04380701, Last updated 12 May 2020, Accessed 6 July 2020.

[239] Clinicaltrials.gov, Safety and Immunity of Covid19 aAPC Vaccine. https://clinicaltrials.gov/ct2/show/ NCT04299724, Last updated 9 March 2020, Accessed 6 July 2020.

[240] Clinicaltrials.gov, Randomized Clinical Trial for the Prevention of SARS-CoV-2 Infection (COVID-19) in Healthcare Personnel (EPICOS). https://clinicaltrials.gov/ct2/show/NCT04334928, Last updated 28 April 2020, Accessed 6 July 2020.

[241] Clinicaltrials.gov, GLS-1200Topical Nasal Spray to Prevent SARS-CoV-2 Infection (COVID-19) in Health Care Personnel. https://clinicaltrials.gov/ct2/ show/NCT04408183, Last updated 4 June 2020, Accessed 6 July 2020.

[242] Clinicaltrials.gov, COVID-19: Povidone-Iodine Intranasal Prophylaxis in Front-line Healthcare Personnel and Inpatients (PIIPPI). https://clinicaltrials.gov/ ct2/show/NCT04364802, Last updated 14 May 2020, Accessed 6 July 2020.

[243] Clinicaltrials.gov, Phase 3 Study to Determine if RTB101 Reduces the Severity of COVID- 
19 in Older Adults Residing in Nursing Homes. https://clinicaltrials.gov/ct2/show/NCT04409327, Last updated 1 June 2020, Accessed 6 July 2020.

[244] Clinicaltrials.gov, Investigating Otilimab in Patients With Severe Pulmonary COVID-19 Related Disease (OSCAR). https://clinicaltrials.gov/ct2/show/NCT04376684, Last updated 21 July 2020, Accessed 6 July 2020.

[245] Clinicaltrials.gov, Mavrilimumab to Reduce Progression of Acute Respiratory Failure in COVID-19 Pneumonia and Systemic Hyper-inflammation. https://clinicaltrials.gov/ct2/show/NCT04399980, Last updated 14 July 2020, Accessed 6 July 2020.

[246] Clinicaltrials.gov, Observational Study, Use of Canakinumab Administered Subcutaneously in the Treatment COVID-19 Pneumonia. https://www.clinicaltrials. gov/ct2/show/NCT04348448, Last updated 16 April 2020, Accessed 6 July 2020.

[247] Clinicaltrials.gov, A Double-blind, Placebo-controlled Clinical Trial of Fluvoxamine for Symptomatic Individuals With COVID-19 Infection (STOP COVID). https://clinicaltrials.gov/ct2/show/NCT04342663, Last updated 19 May 2020, Accessed 6 July 2020.

[248] Clinicaltrials.gov, Proof of Concept Study to Evaluate the Safety Profile of Plitidepsin in Patients With COVID-19 (APLICOV-PC). https://clinicaltrials.gov/ ct2/show/NCT04382066, Last updated 2 July 2020, Accessed 6 July 2020.

[249] Clinicaltrials.gov, Single-Blind Study of a Single Dose of Peginterferon Lambda-1a Compared With Placebo in Outpatients With Mild COVID-19 (COVID-Lambda). https://clinicaltrials.gov/ct2/show/NCT04331899, Last updated 18 June 2020, Accessed 6 July 2020.

[250] Clinicaltrials.gov, Treatment With CSL312 in Adults With Coronavirus Disease 2019 (COVID-19). https://clinicaltrials.gov/ct2/show/NCT04409509, Last updated 8 July 2020, Accessed 6 July 2020.

[251] Clinicaltrialsarena.com, Sun Pharma trials plant-derived drug to treat Covid-19. https://www.clinicaltrialsarena. com/news/sun-pharma-covid-19-trial/, Last updated 8 June 2020, Accessed 6 July 2020.

[252] Cao B, Wang Y, Wen D, Liu W, Wang J, Fan G, Ruan L, Song B, Cai Y, Wei M, Li X, Xia J, Chen N, Xiang J, Yu T, Bai T, Xie X, Zhang L, Li C, Yuan Y, Chen H, Li H, Huang H, Tu S, Gong F, Liu Y, Wei Y, Dong C, Zhou F, Gu X, Xu J, Liu Z, Zhang Y, Li H, Shang L, Wang K, Li K, Zhou X, Dong X, Qu Z, Lu S, Hu X, Ruan S, Luo S, Wu J, Peng L, Cheng F, Pan L, Zou J, Jia C, Wang J, Liu X, Wang S, Wu X, Ge Q, He J, Zhan H, Qiu F, Guo L, Huang C, Jaki T, Hayden FG, Horby PW, Zhang D, Wang C (2020) A trial of Lopinavir-Ritonavir in adults hospitalized with severe Covid-19. N Eng J Med 382, 1787-1799.

[253] Clinicaltrials.gov, Treatments for COVID-19: Canadian Arm of the SOLIDARITY Trial (CATCO). https://clinicaltrials.gov/ct2/show/NCT04330690, Last updated 23 July 2020, Accessed 6 July 2020.

[254] Clinicaltrials.gov, Evaluating and Comparing the Safety and Efficiency of ASC09/Ritonavir and Lopinavir/Ritonavir for Novel Coronavirus Infection. https://clinicaltrials.gov/ct2/show/NCT04261907, Last updated 10 February 2020, Accessed 6 July 2020.

[255] Clinicaltrials.gov, A Prospective/Retrospective, Randomized Controlled Clinical Study of Antiviral Therapy in the 2019-nCoV Pneumonia. https://clinicaltrials.gov/ct2/show/NCT04255017, Last updated 17 March 2020, Accessed 6 July 2020.
[256] Lian N, Xie H, Lin S, Huang J, Zhao J, Lin Q (2020) Umifenovir treatment is not associated with improved outcomes in patients with coronavirus disease 2019: A retrospective study. Clin Microbiol Infec 26, 917921.

[257] Clinicaltrials.gov, COVID-19 First In Human Study to Evaluate Safety, Tolerability, and Pharmacokinetics of EIDD-2801 in Healthy Volunteers. https://clinicaltrials.gov/ct2/show/NCT04392219, Last updated 25 June 2020, Accessed 6 July 2020.

[258] Clinicaltrials.gov, The Efficacy of Ivermectin and Nitazoxanide in COVID-19 Treatment. https://clinicaltrials. gov/ct2/show/NCT04351347, Last updated 22 June 2020, Accessed 6 July 2020.

[259] Clinicaltrials.gov, Ivermectin and Nitazoxanide Combination Therapy for COVID-19. https://clinicaltrials.gov/ct2/show/NCT04360356, Last updated 24 April 2020, Accessed 6 July 2020.

[260] Clinicaltrials.gov, Hydroxychloroquine and Nitazoxanide Combination Therapy for COVID-19. https:// clinicaltrials.gov/ct2/show/NCT04361318, Last updated 24 April 2020, Accessed 6 July 2020.

[261] Clinicaltrials.gov, Efficacy and Safety of Nitazoxanide $600 \mathrm{mg}$ TID for the Treatment of Hospitalized Patients With COVID-19. https://clinicaltrials.gov/ct2/ show/NCT04423861, Last updated 9 June 2020, Accessed on 6 July 2020.

[262] Clinicaltrials.gov, Phase III DAS181 Lower Tract PIV Infection in Immunocompromised Subjects (Substudy: DAS181 for COVID-19): RCT Study. https://clinicaltrials. gov/ct2/show/NCT03808922, Last updated 17 April 2020, Accessed 6 July 2020.

[263] Clinicaltrials.gov, DAS181 for Patients With Severe Hospitalized Flu and SAD-RVs (COVID-19) (STOP-Flu). https://clinicaltrials.gov/ct2/show/NCT04298060, Last updated 9 July 2020, Accessed 29 July 2020.

[264] Clinicaltrials.gov, Study to Evaluate the Safety and Antiviral Activity of Remdesivir (GS-5734 ${ }^{\mathrm{TM}}$ ) in Participants With Severe Coronavirus Disease (COVID-19). https://clinicaltrials.gov/ct2/show/NCT04292899, Last updated 27 July 2020, Accessed 29 July 2020.

[265] Clinicaltrials.gov, Multicenter, Retrospective Study of the Effects of Remdesivir in the Treatment of Severe Covid19 Infections (REMDECO-19). https://clinicaltrials. gov/ct2/show/NCT04365725, Last updated 13 May 2020, Accessed 6 July 2020.

[266] Clinicaltrials.gov, A Study to Evaluate the Safety, Pharmacokinetics and Antiviral Effects of Galidesivir in Yellow Fever or COVID-19. https://clinicaltrials. gov/ct2/show/NCT03891420, Last updated 23 July 2020, Accessed 29 July 2020.

[267] Clinicaltrials.gov, Study to Evaluate the Safety and Efficacy of VIRAZOLE ${ }^{\circledR}$ in Hospitalized Adult Participants With Respiratory Distress Due to COVID-19. https://clinicaltrials.gov/ct2/show/NCT04356677, Last updated 13 July 2020, Accessed 29 July 2020.

[268] Clinicaltrials.gov, The Phase 2 Study to Evaluate the Safety and Efficacy of Clevudine in Patients With Moderate COVID-19. https://clinicaltrials.gov/ct2/show/ NCT04347915, Last updated 20 June 2020, Accessed 6 July 2020.

[269] Clinicaltrials.gov, FAvipiravir and HydroxyChloroquine Combination Therapy (FACCT). https://clinicaltrials.gov/ct2/show/NCT04392973, Last updated 28 July 2020, Accessed 29 July 2020. 
[270] Clinicaltrials.gov, Clinical Study To Evaluate The Performance And Safety Of Favipiravir in COVID-19. https://clinicaltrials.gov/ct2/show/NCT04336904, Last updated 8 April 2020, Accessed 6 July 2020.

[271] Clinicaltrials.gov, Favipiravir Therapy in Adults With Mild COVID-19 (Avi-Mild). https://clinicaltrials.gov/ ct2/show/NCT04464408, Last updated 9 July 2020, Accessed 29 July 2020.

[272] Clinicaltrials.gov, Study of the Use of Favipiravir in Hospitalized Subjects With COVID-19. https://clinicaltrials. gov/ct2/show/NCT04358549, Last updated 29 July 2020, Accessed 29 July 2020.

[273] Viriom, Viriom Initiates Phase 2 Clinical Study of Elsulfavirine for Treatment of COVID-19. https://www.viriom.com/news/2020/4/8/viriom-initiatesphase-2-clinical-study-of-elsulfavirine-for-treatment-ofcovid-19, Last updated 8 April 2020, Accessed 6 July 2020.

[274] Clinicaltrials.gov, A Clinical Trial for Azvudine in the Treatment of Novel Coronavirus Pneumonia (COVID19). https://clinicaltrials.gov/ct2/show/NCT04425772, Last updated 11 June 2020, Accessed 6 July 2020.

[275] Clinicaltrials.gov, Zinc With Chloroquine/Hydroxychloroquine in Treatment of COVID-19. h https:// clinicaltrials.gov/ct2/show/NCT04447534, Last updated 25 June 2020, Accessed 6 July 2020.

[276] Clinicaltrials.gov, Chloroquine/Hydroxychloroquine Prevention of Coronavirus Disease (COVID-19) in the Healthcare Setting (COPCOV). https://clinicaltrials. gov/ct2/show/NCT04303507, Last updated 15 July 2020, Accessed 29 July 2020.

[277] Clinicaltrials.gov, Bevacizumab in Severe or Critically Severe Patients With COVID-19 Pneumonia-RCT (BESTRCT). https://clinicaltrials.gov/ct2/show/NCT04305106, Last updated 26 March 2020, Accessed 6 July 2020.

[278] Clinicaltrials.gov, Bevacizumab in Severe or Critical Patients With COVID-19 Pneumonia (BEST-CP). https://clinicaltrials.gov/ct2/show/NCT04275414, Last updated 3 April 2020, Accessed 6 July 2020.

[279] Clinicaltrials.gov, A Study of the Safety and Efficacy of Ciclesonide in the Treatment of Non-hospitalized COVID-19 Patients. https://clinicaltrials.gov/ct2/show/ NCT04377711, Last updated 11 June 2020, Accessed 6 July 2020

[280] Clinicaltrials.gov, Multi-site Adaptive Trials for COVID19. https://clinicaltrials.gov/ct2/show/NCT04370262, Last updated 16 June 2020, Accessed 6 July 2020.

[281] Clinicaltrials.gov, Safety, Tolerability, and Efficacy of Anti-Spike (S) SARS-CoV-2 Monoclonal Antibodies for Hospitalized Adult Patients With COVID-19. https://clinicaltrials.gov/ct2/show/NCT04426695, Last updated 24 July 2020, Accessed 29 July 2020.

[282] Clinicaltrials.gov, Safety, Tolerability, and Efficacy of Anti-Spike (S) SARS-CoV-2 Monoclonal Antibodies for the Treatment of Ambulatory Adult Patients With COVID19 https://clinicaltrials.gov/ct2/show/NCT04425629, Last updated 27 July 2020, Accessed 6 July 2020.

[283] Clinicaltrials.gov, Tolerability, Safety, Pharmacokinetic Profile and Immunogenicity of a Recombinant Humanized Anti-SARS-CoV-2 Monoclonal Antibody (JS016) for Injection in Chinese Health Subjects. https://clinicaltrials.gov/ct2/show/NCT04441918, Last updated 22 June 2020, Accessed 6 July 2020.

[284] Clinicaltrials.gov, A Study of LY3819253 (LYCoV555) in Participants Hospitalized for COVID-19.
https://clinicaltrials.gov/ct2/show/NCT04411628, Last updated 7 July 2020, Accessed 29 July 2020.

[285] Clinicaltrials.gov, Safety of TY027, a Treatment for COVID-19, in Humans. https://clinicaltrials.gov/ct2/ show/NCT04429529, Last updated 12 June 2020, Accessed 6 July 2020.

[286] Clinicaltrials.gov, Amiodarone or Verapamil in COVID-19 Hospitalized Patients With Symptoms (ReCOVery-SIRIO). https://clinicaltrials.gov/ct2/show/ NCT04351763, Last updated 17 April 2020, Accessed 6 July 2020.

[287] Clinicaltrials.gov, Safety and Efficacy of AT-527 in Subjects With Moderate Coronavirus Disease (COVID-19). https://www.clinicaltrials.gov/ct2/show/NCT04396106, Last updated 24 July 2020, Accessed 29 July 2020.

[288] Biospace, Mayo Clinic preparing to Commence Phase II FDA clinical trial for the treatment of COVID-19 with Vicromax. https://www.biospace.com/article/releases/ma yo-clinic-preparing-to-commence-phase-ii-fda-clinicaltrial-for-the-treatment-of-covid-19-with-vicromax-/, Last updated 16 April 2020, Accessed 6 July 2020.

[289] Clinicaltrials.gov, Desidustat in the Management of COVID-19 Patients. https://clinicaltrials.gov/ct2/show/ NCT04463602, Last updated 24 July 2020, Accessed 29 July 2020.

[290] Clinicaltrials.gov, Hypertension in Patients Hospitalized With COVID-19 (HT-COVID19). https://clinicaltrials. gov/ct2/show/NCT04318301, Last updated 29 April 2020, Accessed 6 July 2020.

[291] Clinicaltrials.gov, Efficacy and Safety of Itolizumab in COVID-19 Complications. https://clinicaltrials.gov/ct2/ show/NCT04475588, Last updated 17 July 2020, Accessed 29 July 2020.

[292] Clinicaltrials.gov, Study to Evaluate the Safety and Efficacy of ATYR1923 In Patients With Severe Pneumonia Related to COVID-19. https://clinicaltrials.gov/ct2/ show/NCT04412668, Last updated 18 June 2020, Accessed 6 July 2020.

[293] Clinicaltrials.gov, A Study of Auxora in Patients With Severe COVID-19 Pneumonia. https://clinicaltrials. gov/ct2/show/NCT04345614, Last updated 22 July 2020, Accessed 29 July 2020.

[294] Clinicaltrials.gov, Clinical Impact of BACTEK-R in Subject With Mild Pneumonia Due to COVID-19 Infection. https://clinicaltrials.gov/ct2/show/NCT04363814, Last updated 30 June 2020, Accessed 6 July 2020.

[295] Clinicaltrials.gov, Evaluation of Ganovo (Danoprevir) Combined With Ritonavir in the Treatment of SARSCoV-2 Infection. https://clinicaltrials.gov/ct2/show/ NCT04291729, Last updated 13 April 2020, Accessed 6 July 2020

[296] Clinicaltrials.gov, Ruxolitinib Managed Access Program (MAP) for Patients Diagnosed With Severe/Very Severe COVID-19 Illness. https://clinicaltrials.gov/ct2/ show/NCT04337359, Last updated 9 July 2020, Accessed 29 July 2020.

[297] Clinicaltrials.gov, TOFAcitinib in SARS-CoV2 Pneumonia. https://clinicaltrials.gov/ct2/show/NCT04332042, Last updated 2 April July 2020, Accessed 29 July 2020.

[298] Clinicaltrials.gov, Application of Desferal to Treat COVID-19. https://clinicaltrials.gov/ct2/show/NCT04 333550, Last updated 4 May 2020, Accessed 29 July 2020.

[299] Clinicaltrials.gov, Clinical Study of Anti-CD147 Humanized Meplazumab for Injection to Treat With 
2019-nCoV Pneumonia. https://clinicaltrials.gov/vct2/ show/NCT04275245, Last updated 19 February 2020, Accessed 6 July 2020.

[300] Clinicaltrials.gov, Efficacy and Safety of Darunavir and Cobicistat for Treatment of COVID-19 (DC-COVID-19). https://clinicaltrials.gov/ct2/show/NCT04252274, Last updated 13 April 2020, Accessed 6 July 2020.

[301] Clinicaltrials.gov, A Study to Evaluate the Safety and Efficacy of MSTT1041A (Astegolimab) or UTTR1147A in Patients With Severe COVID-19 Pneumonia (COVASTIL). https://clinicaltrials.gov/ct2/show/NCT04386 616, Last updated 29 July 2020, Accessed 6 July 2020.

[302] Clinicaltrials.gov, A Study of APL-9 in Adults With Mild to Moderate ARDS Due to COVID-19. https://clinicaltrials.gov/ct2/show/NCT04402060, Last updated 9 June 2020, Accessed 6 July 2020.

[303] Clinicaltrials.gov, Conestat Alfa in the Prevention of Severe SARS-CoV-2 Infection in Hospitalized Patients With COVID-19. https://clinicaltrials.gov/ ct2/show/NCT04414631, Last updated 8 July 2020, Accessed 29 July 2020.

[304] Clinicaltrials.gov, Study of TJ003234 (Anti-GM-CSF Monoclonal Antibody) in Subjects With Severe Coronavirus Disease 2019 (COVID-19). https://www.clinical trials.gov/ct2/show/NCT04341116, Last updated 14 July 2020, Accessed 29 July 2020.

[305] Clinicaltrials.gov, LSALT Peptide vs. Placebo to Prevent ARDS and Acute Kidney Injury in Patients Infected With SARS-CoV-2 (COVID-19). https://clinicaltrials.gov/ct2/show/NCT04402957, Last updated 16 June 2020, Accessed 6 July 2020

[306] Globenewswire, AB Science granted authorization by ANSM to initiate Phase 2 study evaluating masitinib in combination with isoquercetin for the treatment of COVID-19. https://www.globenewswire.com/newsrelease/2020/05/06/2028392/0/en/AB-Science-grantedauthorization-by-ANSM-to-initiate-Phase-2-studyevaluating-masitinib-in-combination-with-isoquercetinfor-the-treatment-of-COVID-19.html, Last updated 6 May 2020, Accessed 6 July 2020.

[307] Clinicaltrials.gov, A Study of $\mathrm{N}$-acetylcysteine in Patients With COVID-19 Infection. https://clinicaltrials.gov/ct2/ show/NCT04374461, Last updated 2 July 2020, Accessed 29 July 2020.

[308] Clinicaltrials.gov, Safety and Efficacy of Viusid and Asbrip in Hospitalized Patients Infected by SARS-Cov2 With COVID-19. https://clinicaltrials.gov/ct2/show/ NCT04407182, Last updated 21 July 2020, Accessed 29 July 2020.

[309] Clinicaltrials.gov, Study of LAU-7b for the Treatment of COVID-19 Disease in Adults (RESOLUTION). https://clinicaltrials.gov/ct2/show/NCT04417257, Last updated 17 July 2020, Accessed 29 July 2020.

[310] Clinicaltrials.gov, Prazosin to Prevent COVID-19 (PREVENT-COVID Trial) (PREVENT). https://clinical trials.gov/ct2/show/NCT04365257, Last updated 15 May 2020, Accessed on 6 July 2020.

[311] Clinicaltrials.gov, Etoposide in Patients With COVID19 Infection. https://clinicaltrials.gov/ct2/show/NCT04 356690, Last updated 11 May 2020, Accessed 6 July 2020.

[312] Clinicaltrials.gov, Telmisartan for Treatment of COVID19 Patients. https://clinicaltrials.gov/ct2/show/NCT 04355936, Last updated 23 July 2020, Accessed 29 July 2020.
[313] Clinicaltrials.gov, Clinical Trial to Evaluate CERC-002 in Adults With COVID-19 Pneumonia and Acute Lung Injury. https://clinicaltrials.gov/ct2/show/NCT04412057, Last updated 27 July 2020, Accessed 29 July 2020.

[314] Centerforbiosimilars, Celltrion Drug Is Tested in COVID19 CATALYST Trial. https://www.centerforbiosimilars. com/news/celltrion-drug-is-tested-in-covid19-catalysttrial, Last updated 11 June 2020, Accessed 6 July 2020.

[315] Clinicaltrials.gov, WGS ANALYSIS OF SARS-COV-2 POSITIVE PATIENTS (CALYPSO). https://clinicaltrials. gov/ct2/show/NCT04353401, Last updated 20 April 2020, Accessed 6 July 2020.

[316] Clinicaltrials.gov, A Study for Efficacy and Safety of Live Biotherapeutic MRx4DP0004 to Treat COVID-19. https://clinicaltrials.gov/ct2/show/NCT04363372, Last updated 1 July 2020, Accessed 6 July 2020.

[317] Clinicaltrials.gov, A Evaluation of Activity and Safety of Oral Selinexor in Participants With Severe COVID-19 Infection (Coronavirus). https://clinicaltrials. gov/ct2/show/NCT04349098, Last updated 8 May 2020, Accessed 6 July 2020.

[318] Clinicaltrials.gov, Study of Immunomodulation Using Naltrexone and Ketamine for COVID-19 (SINK COVID19). https://clinicaltrials.gov/ct2/show/NCT04365985, Last updated 2 July 2020, Accessed 6 July 2020.

[319] Clinicaltrials.gov, Covid-19 Infection and Pulmonary Distress Treatment With Zanubrutinib in Hospitalized Participants. https://clinicaltrials.gov/ct2/show/NCT 04382586, Last updated 29 June 2020, Accessed 6 July 2020.

[320] Clinicaltrials.gov, Acalabrutinib Study With Best Supportive Care Versus Best Supportive Care in Subjects Hospitalized With COVID-19. (CALAVI US). https://clinicaltrials.gov/ct2/show/NCT04380688, Last updated 8 July 2020, Accessed 29 July 2020.

[321] Clinicaltrials.gov, Acalabrutinib Study With Best Supportive Care Versus Best Supportive Care in Subjects Hospitalized With COVID-19. (CALAVI). https://clinicaltrials.gov/ct2/show/NCT04346199, Last updated 27 July 2020, Accessed 29 July 2020.

[322] Clinicaltrials.gov, Dociparstat for the Treatment of Severe COVID-19 in Adults at High Risk of Respiratory Failure. https://clinicaltrials.gov/ct2/show/NCT04389840, Last updated 28 July 2020, Accessed 29 July 2020.

[323] Clinicaltrials.gov, Sargramostim in Patients With Acute Hypoxic Respiratory Failure Due to COVID-19 (SARPAC) (SARPAC). https://clinicaltrials.gov/ct2/ show/NCT04326920, Last updated 24 April 2020, Accessed 6 July 2020.

[324] Clinicaltrials.gov, ABX464 in Treating Inflammation and Preventing Acute Respiratory Failure in Patients With COVID-19 (Mir-Age). https://clinicaltrials.gov/ct2/ show/NCT04393038, Last updated 20 July 2020, Accessed 29 July 2020.

[325] Clinicaltrials.gov, Clazakizumab (Anti-IL- 6 Monoclonal) Compared to Placebo for COVID19 Disease. https://clinicaltrials.gov/ct2/show/NCT04348500, Last updated 5 June 2020, Accessed 6 July 2020.

[326] Clinicaltrials.gov, Anti-Interleukin-8 (Anti-IL-8) for Patients With COVID-19. https://clinicaltrials. gov/ct2/show/NCT04347226, Last updated 1 May 2020, Accessed 6 July 2020.

[327] Clinicaltrials.gov, A Study to Evaluate the Efficacy and Safety of Sirukumab in Confirmed Severe or 
Critical Confirmed Coronavirus Disease (COVID)-19. https://clinicaltrials.gov/ct2/show/NCT04380961, Last updated 20 July 2020, Accessed 29 July 2020.

[328] Clinicaltrials.gov, A Study to Evaluate the Safety and Efficacy of Tocilizumab in Patients With Severe COVID-19 Pneumonia (COVACTA). https://clinicaltrials. gov/ct2/show/NCT04320615, Last updated 29 June 2020, Accessed 6 July 2020.

[329] Clinicaltrials.gov, Tocilizumab in COVID-19 Pneumonia (TOCIVID-19) (TOCIVID-19). https://clini caltrials.gov/ct2/show/NCT04317092, Last updated 13 July 2020, Accessed 29 July 2020.

[330] Clinicaltrials.gov, Efficacy of Tocilizumab in Modifying the Inflammatory Parameters of Patients With COVID-19 (COVITOZ-01) (COVITOZ-01). https://clinicaltrials.gov/ct2/show/NCT04435717, Last updated 17 June 2020, Accessed 6 July 2020.

[331] Clinicaltrials.gov, A RCT - Safety \& Efficacy of Tocilizumab - Tx of Severe COVID-19: ARCHITECTS (ARCHITECTS). https://clinicaltrials.gov/ct2/ show/NCT04412772, Last updated 2 June 2020, Accessed 6 July 2020.

[332] Clinicaltrials.gov, $\mathrm{CD} 24 \mathrm{Fc}$ as a Non-antiviral Immunomodulator in COVID-19 Treatment (SACCOVID). https://clinicaltrials.gov/ct2/show/NCT0431 7040, Last updated 29 May 2020, Accessed 6 July 2020.

[333] Clinicaltrials.gov, Evaluation of the Efficacy and Safety of Sarilumab in Hospitalized Patients With COVID-19. https://clinicaltrials.gov/ct2/show/NCT04315298, Last updated 20 July 2020, Accessed 29 July 2020.

[334] Clinicaltrials.gov, Intravenous Aviptadil for Critical COVID-19 With Respiratory Failure (COVID-AIV). https://clinicaltrials.gov/ct2/show/NCT04311697, Last updated 28 July 2020, Accessed 29 July 2020.

[335] Clinicaltrials.gov, Efficacy and Safety of Siltuximab vs. Corticosteroids in Hospitalized Patients With COVID19 Pneumonia. https://clinicaltrials.gov/ct2/show/NCT 04329650, Last updated 17 April 2020, Accessed 6 July 2020.

[336] Clinicaltrials.gov, An Observational Study of the Use of Siltuximab (SYLVANT) in Patients Diagnosed With COVID-19 Infection Who Have Developed Serious Respiratory Complications (SISCO). https://www.clinicaltrials.gov/ct2/show/NCT04322188, Last updated 1 June 2020, Accessed 6 July 2020.

[337] Clinicaltrials.gov, A Clinical Trial of the Efficacy and Safety of Levilimab (BCD-089) in Patients With Severe COVID-19 (CORONA). https://clinicaltrials. gov/ct2/show/NCT04397562, Last updated 11 June 2020, Accessed 6 July 2020.

[338] Clinicaltrials.gov, Study of the Efficacy and Safety of a Single Administration of Olokizumab and RPH-104 With Standard Therapy in Patients With Severe Severe Acute Respiratory Syndrome Coronavirus 2 (SARSCoV-2) Infection (COVID-19). https://clinicaltrials.gov/ ct2/show/NCT04380519, Last updated 8 May 2020, Accessed 6 July 2020.

[339] Clinicaltrials.gov, InterLeukin-7 (CYT107) to Improve Clinical Outcomes in Lymphopenic pAtients With COVID-19 Infection UK Cohort (ILIAD-7-UK). https:// clinicaltrials.gov/ct2/show/NCT04379076, Last updated 15 May 2020, Accessed 6 July 2020.

[340] Clinicaltrials.gov, M5049 Study in Participants With Coronavirus Disease 2019 (COVID-19) Pneumonia (ANEMONE). https://clinicaltrials.gov/ct2/show/NCT
04448756, Last updated 29 May 2020, Accessed 29 July 2020.

[341] Clinicaltrials.gov, Phase 3 Study to Evaluate Efficacy and Safety of Lenzilumab in Hospitalized Patients With COVID-19 Pneumonia. https://clinicaltrials. gov/ct2/show/NCT04351152, Last updated 11 June 2020, Accessed 6 July 2020.

[342] Clinicaltrials.gov, A Study to Assess the Efficacy and Safety of Gimsilumab in Subjects With Lung Injury or Acute Respiratory Distress Syndrome Secondary to COVID-19 (BREATHE). https://www.clinicaltrials. gov/ct2/show/NCT04351243, Last updated 13 July 2020, Accessed 29 July 2020.

[343] Clinicaltrials.gov, Colchicine Coronavirus SARSCoV2 Trial (COLCORONA) (COVID-19). https:// clinicaltrials.gov/ct2/show/NCT04322682, Last updated 11 June 2020, Accessed 6 July 2020.

[344] Clinicaltrials.gov, ODYSSEY: A Study to Investigate the Efficacy of Tradipitant in Treating Severe or Critical COVID-19 Infection. https://clinicaltrials.gov/ct2/ show/NCT04326426, Last updated 20 April 2020, Accessed 6 July 2020.

[345] Clinicaltrials.gov, Open-label, Randomized Study of IFX-1 in Patients With Severe COVID-19 Pneumonia (PANAMO). https://clinicaltrials.gov/ct2/show/ NCT04333420, Last updated 16 April 2020, Accessed 6 July 2020.

[346] Clinicaltrials.gov, Efficacy and Safety of Emapalumab and Anakinra in Reducing Hyperinflammation and Respiratory Distress in Patients With COVID-19 Infection. https://clinicaltrials.gov/ct2/show/results/NCT04324 021, Last updated 9 April 2020, Accessed 6 July 2020.

[347] Clinicaltrials.gov, Study of the Safety of Therapeutic Treatment With an Immunomodulatory Agent (N-803) in Adults With COVID-19. https:// clinicaltrials.gov/ct2/show/NCT04385849, Last updated 1 June 2020, Accessed 6 July 2020.

[348] Clinicaltrials.gov, Safety and Efficacy Trial of Zavegepant* Intranasal for Hospitalized Patients With COVID-19 Requiring Supplemental Oxygen. https://clinicaltrials.gov/ct2/show/NCT04346615, Last updated 1 July 2020, Accessed 6 July 2020.

[349] Clinicaltrials.gov, Study to Evaluate the Efficacy and Safety of Leronlimab for Patients With Severe or Critical Coronavirus Disease 2019 (COVID-19). https://clinicaltrials.gov/ct2/show/NCT04347239, Last updated 11 June 2020, Accessed 6 July 2020.

[350] Clinicaltrials.gov, Fingolimod in COVID-19. https://clinicaltrials.gov/ct2/show/NCT04280588,

Last updated 21 February 2020, Accessed 6 July 2020.

[351] Clinicaltrials.gov, Umbilical Cord(UC)-Derived Mesenchymal Stem Cells(MSCs) Treatment for the 2019novel Coronavirus(nCOV) Pneumonia. https://clinical trials.gov/ct2/show/NCT04269525, Last updated 1 July 2020, Accessed 6 July 2020.

[352] Clinicaltrials.gov, NestaCell ${ }^{\circledR}$ Mesenchymal Stem Cell to Treat Patients With Severe COVID-19 Pneumonia (HOPE). https://clinicaltrials.gov/ct2/show/NCT 04315987, Last updated 9 June 2020, Accessed 6 July 2020.

[353] Zhang C, Huang S, Zheng F, Dai Y (2020) Controversial treatments: An updated understanding of the Coronavirus Disease 2019. J Med Virol, doi: 10.1002/jmv.25788

[354] ClinicalTrials.gov, Treatment of COVID-19 Patients Using Wharton's Jelly-Mesenchymal Stem Cells, 
https://clinicaltrials.gov/ct2/show/NCT04313322, Last updated 18 March 2020, Accessed 6 July 2020.

[355] ClinicalTrials.gov, Treatment With Human Umbilical Cord-derived Mesenchymal Stem Cells for Severe Corona Virus Disease 2019 (COVID-19) -, https://clinicaltrials.gov/ct2/show/NCT04288102, Last updated 18 June 2020, Accessed 6 July 2020.

[356] ClinicalTrials.gov, Safety and Efficacy Study of Allogeneic Human Dental Pulp Mesenchymal Stem Cells to Treat Severe COVID-19 Patients, https://clinicaltrials. gov/ct2/show/NCT04336254, Last updated 7 April 2020, Accessed 6 July 2020.

[357] ClinicalTrials.gov, Clinical Research of Human Mesenchymal Stem Cells in the Treatment of COVID-19 Pneumonia, https://clinicaltrials.gov/ct2/show/NCT04339660, Last updated 9 April 2020, Accessed 6 July 2020.

[358] ClinicalTrials.gov, Novel Coronavirus Induced Severe Pneumonia Treated by Dental Pulp Mesenchymal Stem Cells, https://clinicaltrials.gov/ct2/show/NCT04302519, Last updated 10 March 2020, Accessed 6 July 2020.

[359] ClinicalTrials.gov, Mesenchymal Stem Cell Treatment for Pneumonia Patients Infected With 2019 Novel Coronavirus, https://clinicaltrials.gov/ct2/show/NCT04252118, Last updated 15 April 2020, Accessed 6 July 2020.

[360] ClinicalTrials.gov, Therapy for Pneumonia Patients iInfected by 2019 Novel Coronavirus, https://clinicaltrials. gov/ct2/show/NCT04293692, Last updated 18 March 2020, Accessed 6 July 2020.

[361] ClinicalTrials.gov, Study of Human Umbilical Cord Mesenchymal Stem Cells in the Treatment of Novel Coronavirus Severe Pneumonia, https://clinicaltrials.gov/ ct2/show/NCT04273646, Last updated 18 March 2020, Accessed 6 July 2020.

[362] ClinicalTrials.gov, Cell Therapy Using Umbilical Cord-derived Mesenchymal Stromal Cells in SARSCoV-2-related ARDS, https://clinicaltrials.gov/ct2/show/ NCT04333368, Last updated 21 May 2020, Accessed 6 July 2020.

[363] ClinicalTrials.gov, Stem Cell Educator Therapy Treat the Viral Inflammation Caused by Severe Acute Respiratory Syndrome Coronavirus, https://clinicaltrials.gov/ct2/ show/NCT04299152, Last updated 14 April 2020, Accessed 6 July 2020.

[364] ClinicalTrials.gov, ASC Therapy for Patients With Severe Respiratory COVID-19, https://clinicaltrials. gov/ct2/show/NCT04341610, Last updated 27 May 2020, Accessed 6 July 2020.

[365] ClinicalTrials.gov, A Pilot Clinical Study on Inhalation of Mesenchymal Stem Cells Exosomes Treating Severe Novel Coronavirus Pneumonia, https:// clinicaltrials.gov/ct2/show/NCT04276987, Last updated 27 May 2020, Accessed 6 July 2020.

[366] ClinicalTrials.gov, Intermediate-size Expanded Access Program (EAP), Mesenchymal Stromal Cells (MSC) for Multisystem Inflammatory Syndrome in Children (MISC) Associated With Coronavirus Disease (COVID-19), https://clinicaltrials.gov/ct2/show/NCT04456439, Last updated 2 July 2020, Accessed 6 July 2020.

[367] ClinicalTrials.gov, Piclidenoson for Treatment of COVID19, https://clinicaltrials.gov/ct2/show/NCT04333472, Last updated 14 July 2020, Accessed 14 July 2020.

[368] ClinicalTrials.gov, PRE-VENT Study in Hospitalized Patients With Severe COVID-19 With or Without Cancer, https://clinicaltrials.gov/ct2/show/NCT04404361, Last updated 8 July 2020, Accessed 8 July 2020.
[369] ClinicalTrials.gov, Evaluating AVM0703 for Treatment of COVID-19, https://clinicaltrials.gov/ct2/show/ NCT04366115, Last updated 27 May 2020, Accessed 6 July 2020.

[370] ClinicalTrials.gov, Randomised Evaluation of COVID19 Therapy, https://clinicaltrials.gov/ct2/show/NCT0438 1936, Last updated 7 July 2020, Accessed 7 July 2020.

[371] ClinicalTrials.gov, A Randomized Trial of Anticoagulation Strategies in COVID-19, https://clinicaltrials. gov/ct2/show/NCT04359277, Last updated 23 June 2020, Accessed 6 July 2020.

[372] ClinicalTrials.gov, Baricitinib in Symptomatic Patients Infected by COVID-19: An Open-label, Pilot Study, https://clinicaltrials.gov/ct2/show/NCT04320277, Last updated 22 April 2020, Accessed 6 July 2020.

[373] ClinicalTrials.gov, Safety and Efficacy of Baricitinib for COVID-19, https://clinicaltrials.gov/ct2/show/ NCT04340232, Last updated 6 May 2020, Accessed 6 July 2020.

[374] Bergenbio (nd) Bemcentinib Potential First-in-class AXL Inhibitor, BerGenBio, https://www.bergenbio.com/ pipeline/bemcentinib-melanoma/, Accessed on 6 July 2020.

[375] ClinicalTrials.gov, The Impact of Camostat Mesilate on COVID-19 Infection, https://clinicaltrials.gov/ct2/show/ NCT04321096, Last updated 30 April 2020, Accessed 6 July 2020.

[376] ClinicalTrials.gov, Efficacy of Nafamostat in Covid-19 Patients (RACONA Study), https://clinicaltrials.gov/ct2/ show/NCT04352400, Last updated 20 April 2020, Accessed 6 July 2020.

[377] ClinicalTrials.gov, Double-Blind, Multicenter, Study to Evaluate the Efficacy of PLX PAD for the Treatment of COVID-19, https://clinicaltrials.gov/ct2/show/ NCT04389450, Last updated 29 July 2020, Accessed 29 July 2020.

[378] ClinicalTrials.gov, First in Human SAD and MAD Study of Inhaled TD-0903, a Potential Treatment for ALI Associated With COVID-19, https://clinicaltrials. gov/ct2/show/NCT04350736, Last updated 22 May 2020, Accessed 6 July 2020.

[379] ClinicalTrials.gov, A Study of Trans Crocetin in Patients With Acute Respiratory Distress Syndrome Due to COVID-19 Disease (LEAF-4L7520/4L6715), https:// clinicaltrials.gov/ct2/show/NCT04378920, Last updated 14 May 2020, Accessed 6 July 2020.

[380] ClinicalTrials.gov, A Phase 2 Study to Evaluate Axatilimab for Hospitalized Patients With Respiratory Involvement Secondary to COVID-19, https://clinicaltrials.gov/ct2/show/NCT04415073, Last updated 4 June 2020, Accessed 6 July 2020.

[381] ClinicalTrials.gov, Low-doses Melphalan Inhalation in Patients With COVID-19 (CoronavIrus Disease 2019) Pneumonia, https://clinicaltrials.gov/ct2/show/NCT043 80376, Last updated 12 May 2020, Accessed 6 July 2020.

[382] ClinicalTrials.gov, CORIMUNO19-ECU: Trial Evaluating Efficacy and Safety of Eculizumab, (Soliris) in Patients With COVID-19 Infection, Nested in the CORIMUNO-19 Cohort, https://clinicaltrials.gov/ct2/ show/NCT04346797, Last updated 27 April 2020, Accessed 6 July 2020.

[383] ClinicalTrials.gov, COVID-19 Treatment of Severe Acute Respiratory Syndrome With Veru-111, https://clinicaltrials.gov/ct2/show/NCT04388826, Last updated 23 July 2020, Accessed 23 July 2020. 
[384] Clinicaltrials.gov, Sirolimus Treatment in Hospitalized Patients With COVID-19 Pneumonia. https://clinicaltrials.gov/ct2/show/NCT04341675, Last updated 20 May 2020, Accessed 29 July 2020.

[385] Clinicaltrials.gov, Study of Efficacy and Safety of DV890 in Patients With COVID-19 Pneumonia. https:// clinicaltrials.gov/ct2/show/NCT04382053, Last updated 28 July 2020, Accessed 29 July 2020.

[386] Clinicaltrials.gov, Study of Efficacy and Safety of MAS825 in Patients With COVID-19. https://clinicaltrials.gov/ct2/show/NCT04382651, Last updated 28 July 2020, Accessed 29 July 2020.

[387] Clinicaltrials.gov, Efficacy and Safety Study of BDB-001 in Severe COVID-19 With ALI/ARDS https://clinicaltrials.gov/ct2/show/NCT04449588, Last updated 27 July 2020, Accessed 29 July 2020.

[388] Clinicaltrials.gov, Avdoralimab an Anti-C5aR Antibody, in Patients With COVID-19 Severe Pneumonia (FORCE). https://clinicaltrials.gov/ct2/show/NCT04371367, Last updated 1 May 2020, Accessed 29 July 2020.

[389] Clinicaltrials.gov, Trial of Treatments for COVID-19 in Hospitalized Adults. https://clinicaltrials.gov/ct2/show/ NCT04315948, Last updated 22 July 2020, Accessed 29 July 2020.

[390] Clinicaltrials.gov, Trial of Inhaled Anti-viral (SNG001) for SARS-CoV-2 (COVID-19) Infection. https:// clinicaltrials.gov/ct2/show/NCT04385095, Last updated 12 May 2020, Accessed 29 July 2020.

[391] Clinicaltrials.gov, Evaluation of Safety \& Efficacy of BIO-11006 Inhalation Solution in Patients With ARDS. https://clinicaltrials.gov/ct2/show/NCT03202394, Last updated 25 June 2020, Accessed 29 July 2020.

[392] Clinicaltrials.gov, mulTi-Arm Therapeutic Study in Pre-ICu Patients Admitted With Covid-19 - Repurposed Drugs (TACTIC-R). https://clinicaltrials.gov/ct2/ show/NCT04390464, Last updated 18 May 2020, Accessed 29 July 2020.

[393] Clinicaltrials.gov, Efficacy and Safety Study of IV Ravulizumab in Patients With COVID-19 Severe Pneumonia. https://clinicaltrials.gov/ct2/show/NCT04369469, Last updated 1 July 2020, Accessed 29 July 2020.

[394] Clinicaltrials.gov, Checkpoint Blockade in COVID19 Pandemic. https://clinicaltrials.gov/ct2/show/NCT04 335305, Last updated 9 June 2020, Accessed 29 July 2020.

[395] Clinicaltrials.gov, Trial Evaluating Efficacy and Safety of Nivolumab (Optivo ${ }^{\circledR}$ ) in Patients With COVID19 Infection, Nested in the Corimmuno-19 Cohort. https://clinicaltrials.gov/ct2/show/NCT04343144, Last updated 14 April 2020, Accessed 29 July 2020.

[396] Clinicaltrials.gov, A Study of LY3127804 in Participants With COVID-19. https://clinicaltrials.gov/ct2/ show/NCT04342897, Last updated 17 July 2020, Accessed 29 July 2020.

[397] Clinicaltrials.gov, Inhaled Iloprost for Suspected COVID-19 Respiratory Failure. https://clinicaltrials. gov/ct2/show/NCT04445246, Last updated 24 June 2020, Accessed 29 July 2020.

[398] Clinicaltrials.gov, Immunoregulatory Therapy for 2019nCoV. https://clinicaltrials.gov/ct2/show/NCT04268537, Last updated 13 February 2020, Accessed 29 July 2020.

[399] Clinicaltrials.gov, The Use of PUL-042 Inhalation Solution to Reduce the Severity of COVID-19 in Adults Positive for SARS-CoV-2 Infection. https://clinical trials.gov/ct2/show/NCT04312997, Last updated 19 June 2020, Accessed 29 July 2020.
[400] Clinicaltrials.gov, Natural Killer Cell (CYNK-001) Infusions in Adults With COVID-19 (CYNK-001COVID-19). https://clinicaltrials.gov/ct2/show/NCT04 365101, Last updated 17 June 2020, Accessed 29 July 2020.

[401] Clinicaltrials.gov, DUR-928 in Subjects With SARS-CoV-2 With Acute Liver or Kidney Injury. https://clinicaltrials.gov/ct2/show/NCT04447404, Last updated 25 June 2020, Accessed 29 July 2020.

[402] Clinicaltrials.gov, Losartan for Patients With COVID19 Not Requiring Hospitalization. https://clinicaltrials. gov/ct2/show/NCT04311177, Last updated 16 June 2020, Accessed 29 July 2020.

[403] Clinicaltrials.gov, Valsartan for Prevention of Acute Respiratory Distress Syndrome in Hospitalized Patients With SARS-COV-2 (COVID-19) Infection Disease. https://clinicaltrials.gov/ct2/show/NCT04335786, Last updated 9 June 2020, Accessed 29 July 2020.

[404] Clinicaltrials.gov, Dapagliflozin in Respiratory Failure in Patients With COVID-19. https://clinicaltrials. gov/ct2/show/NCT04350593, Last updated 18 June 2020, Accessed 29 July 2020.

[405] Clinicaltrials.gov, Compassionate Use of Opaganib in Patients With Coronavirus Disease 2019 (COVID-19). https://clinicaltrials.gov/ct2/show/NCT04435106, Last updated 17 June 2020, Accessed 29 July 2020.

[406] Clinicaltrials.gov, Recombinant Human Angiotensinconverting Enzyme 2 (rhACE2) as a Treatment for Patients With COVID-19. https://clinicaltrials.gov/ct2/show/ NCT04335136, Last updated 17 July 2020, Accessed 29 July 2020.

[407] Clinicaltrials.gov, Safety and Antiviral Activity of BLD-2660 in COVID-19 Hospitalized Subjects. https:// clinicaltrials.gov/ct2/show/NCT04334460, Last updated 8 July 2020, Accessed 29 July 2020.

[408] Clinicaltrials.gov, A Study to Evaluate the Efficacy, Safety and Tolerability of IMU-838 as Addition to Investigator's Choice of Standard of Care Therapy, in Patients With Coronavirus Disease 19 (COVID-19). https://www. clinicaltrials.gov/ct2/show/NCT04379271, Last updated 1 July 2020, Accessed 29 July 2020.

[409] Clinicaltrials.gov, A Study to Evaluate Efficacy and Safety of PTC299 in Hospitalized Participants With Coronavirus (COVID-19). https://clinicaltrials. gov/ct2/show/NCT04439071, Last updated 22 July 2020, Accessed 29 July 2020.

[410] Clinicaltrials.gov, Trial of Imatinib for Hospitalized Adults With COVID-19. https://clinicaltrials. gov/ct2/show/NCT04394416, Last updated 4 June 2020, Accessed 29 July 2020.

[411] Clinicaltrials.gov, Convalescent Plasma Therapy From Recovered Patients to Treat Severe SARS-CoV-2 Disease (CONCOVID Study). https://clinicaltrials.gov/ ct2/show/NCT04342182, Last updated 18 May 2020, Accessed 29 July 2020.

[412] Clinicaltrials.gov, Rapid SARS-CoV-2 IgG Antibody Testing in High Risk Healthcare Workers. https://clinicaltrials.gov/ct2/show/NCT04334876, Last updated 6 April 2020, Accessed 29 July 2020.

[413] Clinicaltrials.gov, Expanded Access to Convalescent Plasma for the Treatment of Patients With COVID-19. https://clinicaltrials.gov/ct2/show/NCT04338360, Last updated 8 April 2020, Accessed 29 July 2020.

[414] Clinicaltrials.gov, Safety in Convalescent Plasma Transfusion to COVID-19. https://clinicaltrials.gov/ct2/ 
show/NCT04333355, Last updated 28 July 2020, Accessed 29 July 2020.

[415] Clinicaltrials.gov, COVID-19 Convalescent Plasma. https://clinicaltrials.gov/ct2/show/NCT04327349, Last updated 31 March 2020, Accessed 29 July 2020.

[416] Clinicaltrials.gov, Anti-SARS-CoV-2 Inactivated Convalescent Plasma in the Treatment of COVID-19. https://clinicaltrials.gov/ct2/show/NCT04292340, Last updated 3 March 2020, Accessed on 29 July 2020.

[417] Clinicaltrials.gov, Study Testing Convalescent Plasma vs Best Supportive Care. https://clinicaltrials.gov/ct2/ show/NCT04333251, Last updated 6 April 2020, Accessed 29 July 2020.

[418] Clinicaltrials.gov, Convalescent Plasma for Patients With COVID-19: A Randomized, Open Label, Parallel, Controlled Clinical Study. https://clinicaltrials.gov/ ct2/show/NCT04332835, Last updated 28 April 2020, Accessed 29 July 2020.

[419] Clinicaltrials.gov, Efficacy and Safety Human Coronavirus Immune Plasma (HCIP) vs. Control (SARS-CoV-2 Non-immune Plasma) Among Adults Exposed to COVID19. https://clinicaltrials.gov/ct2/show/study/NCT043238 00, Last updated 17 July 2020, Accessed 29 July 2020.

[420] Clinicaltrials.gov, Investigating Effect of Convalescent Plasma on COVID-19 Patients Outcome: A. https://clinicaltrials.gov/ct2/show/NCT04327349, Last updated 31 March 2020, Accessed 29 July 2020.
[421] Clinicaltrials.gov, Convalescent Plasma to Limit Coronavirus Associated Complications. https://clinicaltrials. gov/ct2/show/NCT04325672, Last updated 8 April 2020, Accessed 29 July 2020.

[422] Barone P, DeSimone RA (2020) Convalescent plasma to treat coronavirus disease 2019 (COVID-19): Considerations for clinical trial design. Transfusion 60, 1123-1127.

[423] Clinicaltrials.gov, A Study to Evaluate the Safety, Tolerability and Pharmacokinetics (PK) of TAK-981 in Adult Participants With Advanced or Metastatic Solid Tumors or Relapsed/Refractory Hematologic Malignancies and in a Subset With Coronavirus Disease 2019 (COVID-19). https://clinicaltrials.gov/ct2/show/NCT03648372, Last updated 22 May 2020, Accessed 29 July 2020.

[424] Clinicaltrials.gov, NA-83, Atazanavir and Dexamethasone Combination Therapy for the Treatment of COVID-19 Infection. https://clinicaltrials.gov/ct2/show/ NCT04452565, Last updated 13 July 2020, Accessed 29 July 2020.

[425] Clinicaltrials.gov, Study of the Efficacy and Safety of Intravenous Pamrevlumab, in Hospitalized Patients With Acute COVID-19 Disease. https://clinicaltrials.gov/ ct2/show/NCT04432298, Last updated 24 June 2020, Accessed 29 July 2020. 